\title{
Imaging prototypical aromatic molecules on insulating surfaces: a review
}

\author{
R Hoffmann-Vogel ${ }^{\circ}$ \\ Physikalisches Institut, Karlsruhe Institute of Technology (KIT), D-76131 Karlsruhe, Germany \\ Institut für Angewandte Physik, Karlsruhe Institute of Technology (KIT), D-76131 Karlsruhe, Germany \\ E-mail: r.hoffmann@kit.edu
}

\begin{abstract}
Insulating substrates allow for in-plane contacted molecular electronics devices where the molecule is in contact with the insulator. For the development of such devices it is important to understand the interaction of molecules with insulating surfaces. As substrates, ionic crystals such as $\mathrm{KBr}, \mathrm{KCl}, \mathrm{NaCl}$ and $\mathrm{CaF}_{2}$ are discussed. The surface energies of these substrates are small and as a consequence intrinsic properties of the molecules, such as molecule-molecule interaction, become more important relative to interactions with the substrates. As prototypical molecules, three variants of graphene-related molecules are used, pentacene, $\mathrm{C}_{60}$ and PTCDA. Pentacene is a good candidate for molecular electronics applications due to its high charge carrier mobility. It shows mainly an upright standing growth mode and the morphology of the islands is strongly influenced by dewetting. A new second flat-lying phase of the molecule has been observed. Studying the local work function using the Kelvin method reveals details such as line defects in the center of islands. The local work function differences between the upright-standing and flat-lying phase can only be explained by charge transfer that is unusual on ionic crystalline surfaces. $\mathrm{C}_{60}$ nucleation and growth is explained by loosely bound molecules at kink sites as nucleation sites. The stability of $\mathrm{C}_{60}$ islands as a function of magic numbers is investigated. Peculiar island shapes are obtained from unusual dewetting processes already at work during growth, where molecules 'climb' to the second molecular layer.

PTCDA is a prototypical semiconducting molecule with strong quadrupole moment. It grows in the form of elongated islands where the top and the facets can be molecularly resolved. In this way the precise molecular arrangement in the islands is revealed.
\end{abstract}

Keywords: self-assembly of molecules, dewetting of molecules, interactions, scanning force microscopy, surfaces of wide-band gap insulators

\section{Introduction}

In molecular electronics studies the main idea is to replace existing semiconductor functional units such as diodes and transistors by molecules having functionalities by their chemical design. This can be done using single crystals [1], using thin films, e.g. in organic light emitting diodes, or, pushing miniaturization to the ultimate limits, even using a single molecule [2]. Such ideas have triggered a variety of studies of molecular layers and single molecules on surfaces with scanning tunneling microscopy (STM) [3-8].

In such STM studies, the adsorption geometry and possible functionality of the molecule is investigated. When the STM is approached to even closer distances, molecules are contacted 
by STM in a two-terminal arrangement [9-13]. However, it is not simple to produce a large number of such STM contacted molecules on a small area, the technique is non-scalable. Also, the arrangement is affected by thermal drift. A third contact needed to build a transistor is difficult to integrate. Amongst the efforts to connect electric leads to molecules [14], an alternative approach is to use several probes to study electronic transport through the molecule in an in-plane configuration [15]. Such in-plane contacts are much more stable than STM configuration [16]. For this approach, either free-standing contacts or insulating substrates are needed. For these technological reasons, there is a clear need to understand the interaction of molecules with insulating substrates.

The interaction of molecules with insulating surfaces is also of particular fundamental interest because the surface energies of insulators are in general small compared to metals. Therefore, single molecules are more mobile and must often be studied at low temperatures to be immobilized. The main components governing molecule-substrate interaction are often the van-der-Waals and the electrostatic interaction. These are of fundamentally different nature on insulators compared to metals. Because the interaction with the substrate is weak, molecule-molecule interaction comes to the foreground and can more easily be studied. A rich variety of island shapes can be observed. These island shapes are in addition influenced by kinetic effects depending on the barrier height of the diffusion processes involved. As a result the molecular arrangements are in many cases peculiar as will be shown for $\mathrm{C}_{60}$. A key problem is to tailor the structures of molecular conformations on the surface, because electronic transport is strongly related to the structure [17]. In general, for long molecules two main orientations are observed: upright standing or lying configurations. Pentacene has-due to its shape-highly anisotropic conduction properties and it is therefore important to gain control over its growth. It has been possible to use kinetic effects to obtain some control over the growth direction [18]. If the intermolecular interaction is temperature dependent this allows to tune the growth as has been done for example for Sn-phtalocyanine which grows in several ordered phases [19]. Dewetting is an expression of the molecule-surface interaction and kinetics and has been studied in detail [20, 21].

Scanning force microscopy (SFM) is a method of choice to study molecular arrangements on insulating surfaces, because it can easily be used to study island shapes in the micrometer and nanometer range through the local measurement of forces such as van-der-Waals and electrostatic forces [22]. In addition, SFM is ideally suited to study insulating surfaces with atomic resolution [23-25]. This method also provides molecular resolution not only on metals [26, 27], but also on bulk insulators [28-33], for an overview, see also [34, 35]. If stiff force sensors and small oscillation amplitudes are used, the method even yields atomic resolution on molecules [36, 37].

Another key problem for molecular electronics is to understand the electronic properties of the molecules in contact with insulators: a contact to another material often leads to charge transfer, shifts the Fermi level of the molecule and as a consequence strongly influences electronic transport. This has been the subject of intense study [38-41]. The Kelvin method, where the contact potential difference between tip and surface is nullified, allows to determine local variations of the contact potential. It has been shown that the value of the work function depends on the orientation of the molecule [42] and that additional structures of electrostatic nature can be found on molecular islands that help to better understand the molecular arrangement [43] and charge trapping [44-46]. Charge transfer is often related to electronic hybridization between the molecule and the surface. Electronic hybridization should also be considered to understand electronic transport [47]. Placing the molecule on insulating substrates allows to study charge injection into molecular islands [48, 49].

This review centers on three aromatic molecules all of which can be viewed as variants of graphene: pentacene, and $\mathrm{C}_{60}$, as graphitic compounds, and PTCDA, as a graphene variant with additional oxygen atoms. All of them have sizes on the order of a nanometer. We will mainly focus on ionic crystals as substrates and on experiments performed under ultra-high vacuum conditions where molecular resolution was obtained. We discuss both the large-scale structure of molecular islands created by growth and dewetting, as well as the molecular arrangement and the electronic properties as determined with Kelvin probe SFM.

\section{Self-assembly of molecular layers}

\subsection{Overall thermodynamic considerations}

Self-assembly is defined as the formation of structures from pre-existing components (i.e. molecules). This formation should be reversible and should be controlled by the design of the pre-existing components [50]. These principles have been employed in solution chemistry and one has made use of hydrogen bonding, donor-acceptor interactions, and metal coordination interactions for controlling the processes and holding the components together [51]. In 1987, Donald J. Cram, Jean-Marie Lehn and Charles J Pedersen were awarded the Nobel prize for their development and use of molecules with structure-specific interactions of high selectivity. It has been stressed that self-assembly allows to encode information on the building blocks and their mutual interaction in the formed structures [50]. Here, we discuss how the concept of self-assembly has been extended from solution chemistry to the vacuum [3] and in particular to ionic crystalline surfaces [52]. We keep the required condition that the components must be able to move with respect to one another to explore several configurations, make and break bonds and find equilibrium [50].

Self-assembly implies to use thermodynamics to obtain particular structures of molecules on surfaces. The results of the thermodynamic ordering process should be controlled by controlling the thermodynamic details of the different processes occurring at the surface. Many aspects depend on the energy scales involved which are hierarchic and define which processes are accessible at the temperature of study. Phase transitions and thermally activated processes are readily described in a statistical way. Influencing both the magnitude of energy barriers and relative energies of the states is central 
Table 1. Examples for defects of different dimensions. The dimension mainly determines the energy of the state. After [56].

\begin{tabular}{ll}
\hline Dimension & Structural element \\
\hline 0 & $\begin{array}{l}\text { Point defect: adatom, adsorbed molecule, vacancy, } \\
\text { atoms or molecules at kink sites }\end{array}$ \\
1 & $\begin{array}{l}\text { Atomic or molecular step, wire-like molecular } \\
\text { island, domain edge, domain boundary, phase } \\
\text { boundary, dislocation } \\
\end{array}$ \\
& Superstructure, single molecular layer island, facet \\
\hline
\end{tabular}

in influencing the structures on the surface. As a general rule the rate of a thermodynamical process $f$ is given by

$$
f(T)=\nu_{0} \exp -\frac{\Delta \varepsilon}{\left(k_{\mathrm{B}} T\right)}
$$

where $T$ is the temperature, $\nu_{0}$ is the attempt frequency, $\Delta \varepsilon$ is the energy barrier to overcome, $k_{\mathrm{B}}$ is Boltzmann's constant. Not only the relative energies of the different states are relevant, but energy barriers existing between various states could be used to stabilize non-equilibrium situations: if the energy barriers are small enough to be overcome at experimentally accessible timescales, the relative abundance of the excited state is determined by its energy difference to the ground state and the surface is in thermodynamic equilibrium. If the energy barriers are higher, the surface could be trapped in a kinetically hindered non-equilibrium state.

In thermodynamic equilibrium, a defect with a certain energy required for forming it, has a certain probability to exist given by temperature. Examples are vacancy-adatom (vacancy-adsorbed molecule) pairs, on-surface two-dimensional (lattice) gaseous atoms or molecules in thermodynamic equilibrium with atoms or molecules attached to islands [53], or steps on a flat surface in equilibrium with the flat parts of the surface [54]. This is taken into account by using the Gibbs free energy $\Delta G=\Delta H-T \Delta S$ instead of the formation energy $\Delta \varepsilon$. This has been investigated for dibutyl sulfide molecules at low temperatures [55].

In these considerations the dimensionality of the objects under study plays an important role because it determines the number of chemical bonds and thus has a direct influence on the energy of the state, see table 1 .

\subsection{Hierarchy of energy scales}

For studies of molecules on ionic crystals, generally molecules are deposited on freshly prepared clean surfaces using thermal evaporation. The dissociation energy of the molecules should be the largest energy scale, because the molecules should be evaporated and deposited on the surface without damaging their inner structure. The evaporation energy of molecules generally increases with size. This requirement limits the size of molecules to be studied on surfaces. The situation could be changed when one attempts on-surface synthesis [57, 58].

The second largest energy scale should be desorption from the surface since we require the structures to form on the surface. This review is specifically concerned with insulating surfaces. The surface energies of insulators are usually much smaller than the ones of metals. As a result, molecules generally desorb at much smaller temperatures compared to metals.

Next we assume the presence of islands without discussing here the detailed kinetic process of their formation but rather focus on their structure. These islands have an elastic energy if they are slightly deformed from equilibrium and the local interaction energy with the surface [59]. Their structure is the result of optimization between these two energy contributions which occur at the same energy scale. The elastic energy is given by the molecule-molecule interaction. The local interaction with the surface is related to the energy barrier for diffusion. If the local interaction dominates over the elastic energy of the island, the island's structure is strongly influenced by the substrate resulting in a substrate templating effect [52]. This situation is not often achieved on insulating surfaces. One reason is that the molecules are generally much larger than atomic unit cells and that for geometric reasons it is hard to find suitable adsorption for many molecules in the large unit cells. Another reason is that the diffusion barriers are often small on insulating surfaces. Usually the desorption energy is related to the diffusion barrier for surface diffusion. Since for insulating surfaces, the desorption energy is small, also the diffusion barrier is usually small, an exception is discussed in the section 6.6 where for the $\mathrm{C}_{60}$ on $\mathrm{KBr}(001)$ system the relation of diffusion and desorption energy is different compared to the $\mathrm{C}_{60}$ on graphite system. As a result the surface does not strongly influence the structure of the island often resulting in islands having the bulk crystalline structure. While this is an advantage if the intermolecular interactions are to be studied, it is a disadvantage for keeping the island in contact with the substrate. Often low temperatures are needed. To attach molecules in a more stable way to the substrate, anchoring strategies are used which are discussed below in section 2.3.1 on molecule-substrate interactions.

For rather perfect insulating surfaces, as achieved by cleaving, the island density could be used to estimate the diffusion barrier from the size or density of islands [60, 61]. However, the density could be influenced by the interaction of molecules with atomic scale defects created by interaction with charged particles such as electrons [62]. In the context of molecules on insulating surfaces, templating is achieved by interaction of the molecules with substrate steps. Trapping of molecules by artificial pits has been explored where the pits were also created by electron irradiation [63]. Trapping occurs if the energy barrier for diffusion across steps, the Ehrlich-Schwoebel barrier is large enough, which is often the case [64].

\subsection{Interactions}

2.3.1. Molecule-molecule interactions. For molecular selfassembly, weak interactions between molecules are needed. Traditionally, mostly the van-der-Waals interaction and hydrogen-bonding were used [65-67]. Amongst the forces that govern supramolecular assemblies on insulating surfaces are also the van-der-Waals forces [68], electrostatic contributions such as dipolar forces [69] and hydrogen-bonding [70, 71]. For a comparison of the strength and properties of various interactions, see [52]. The van-der-Waals interaction is non-directional and only 
poorly selective. Hydrogen bonding is anisotropic and highly directional. It results from a charge distribution that favors a hydrogen located between two negatively charged species [72]. It has been believed earlier that the proton takes part in a covalent bonding where the proton is shared between bonded molecules, but this has been disproven. However, the hydrogen bond does have some covalent character [73]. An additional bonding type is the well-known $\pi-\pi$-interaction that leads to a special stacking of aromatic $\pi$-electron systems [74]. In addition intermolecular interactions particular to certain types of atoms have been added. These are not to be confused with covalent bonding. Examples so far mainly used on metal surfaces are the halogen bond [75], the pnicogen bond, where $\mathrm{P}$ and $\mathrm{N}$ are bonded to electron donors/acceptors [76], and the carbon bond [77].

2.3.2. Molecule-substrate interactions. All relevant interactions at the nanoscale result from electromagnetic interactions [78]. These could be put into three different categories [73]: interactions resulting from the charge distribution, interactions resulting from polarizations and interactions resulting from quantum mechanical effects.

Electrostatic forces between point-like objects are longranged and isotropic. The electrostatic interaction between extended objects, however, e.g. in the situation where a molecule approaches an ionic crystalline surface, is generally different. In a simple (toy) model [79] we consider a plane of periodically arranged rigid ions of alternating sign and we consider its interaction with a point-like charge, possibly a molecule. Solving the Laplace equation,

$$
\Delta \Phi(\vec{x})=\rho(\vec{x})
$$

where $\Phi(\vec{x})$ is the electrostatic potential and $\rho(\vec{x})$ the electrostatic charge distribution, becomes easier in two dimensional Fourier space. Since the arrangement of charge is periodic with lattice constants $k_{x}$ in $x$-direction, and $k_{y}$ in $y$-direction, taking the derivative of $\Phi(\vec{x})$ reduces to multiplying by $i k_{x}$ or $i k_{y}$ respectively. Since in the space between the point-like object and the periodic charge arrangement of the surface, there is vacuum, and the interaction must be zero at infinite distances, one obtains

$$
\left(-k_{x}^{2}-k_{y}^{2}+\frac{\partial^{2}}{\partial z^{2}}\right) \Phi(\vec{x})=0 .
$$

With $k=\sqrt{k_{x}^{2}+k_{y}^{2}}$ the solution becomes

$$
\Phi(\vec{x})=\Phi(x, y) \mathrm{e}^{-k z} .
$$

It becomes clear that the forces decay exponentially as a function of distance, with a decay constant related to the lattice constant of the periodic arrangement. As a consequence, the molecules feels a force only when it is approached to within interatomic distances to the surface. These considerations also apply to tip-sample forces measured in SFM experiments [80].

For van-der-Waals interactions at first approximation we expect the main contribution to be uniform on the surface without atomic-scale variations. The interaction laws for various shapes of molecules are discussed in [73]. The above considerations apply, too, but only lead to small variations on top of the uniform background.
In addition to electrostatic and van-der-Waals forces, covalent bonding should be considered. It has been found that molecular electronic orbitals can hybridize with surface-orbitals even for insulating surfaces with a wide band gap [47]. The hybridization was found to be related to the polarizability of the molecule: for strongly polarizable $\mathrm{C}_{60}$, it was much stronger compared to PTCDA. Charging molecular islands could be a route to study charge attachment and stability that is related to electronic hybridization as has been shown for $\mathrm{C}_{60}$ on a $\mathrm{NaCl}(001)$ surface [49].

A route to tailor the interactions of molecules with surfaces is to add anchoring groups with known and strong tip-sample interactions [35, 81]. Three main strategies were identified: choosing an appropriate molecule-surface interaction, matching the substrate with the molecular size and periodicity of the layer and increasing the number of strongly interacting functional groups. In particular it has been stressed that the calcite(104) surface can act as a proton acceptor, making it prone to interaction with acids as has been demonstrated using carboxylic acids.

\subsection{Epitaxy of molecular layers}

Epitaxy occurs if there is a fixed relationship between the crystallographic lattice of an overlayer with that of the substrate. Such relationships occur after minimizing the interaction energy with the surface as well as the elastic energy of the overlayer. Weak molecule-surface interactions together with the large size differences between the molecules and the substrate unit-cells cause unusual types of epitaxy rarely known from studies of pure elements. These types of epitaxy are typically of lower symmetry than the ones known from simpler materials. To discuss these types of epitaxy, it is helpful to put the two lattices into a relation to each other using a matrix

$$
\left(\begin{array}{l}
\vec{b}_{1} \\
\vec{b}_{2}
\end{array}\right)=\left(\begin{array}{ll}
c_{11} & c_{21} \\
c_{12} & c_{22}
\end{array}\right)\left(\begin{array}{l}
\vec{a}_{1} \\
\vec{a}_{2}
\end{array}\right) .
$$

For commensurate structures, the numbers $c_{i j}$ are small integers [59]. For each unit cell, the unit cell of the overlayer is attached to a point of the substrate that is crystallographically equivalent by translation. Usually, these are points of high symmetry. For point-on-line epitaxy, only part of the $c_{i j}$, located in one column, are small integers, see figure 1 . However, since one of the two columns of the matrix $c$ must consist of integers, the lattice points of these structures lie on high-symmetry lines of the substrate. For coincidence structures, at least one of the matrix elements must be an irrational number and neither column must consist of integers. This definition is purely mathematic since on experimental length scales, one cannot determine the difference between rational and irrational numbers. In practice, one assumes a number be irrational if it cannot be represented as a fraction of small integers. In coincidence structures there exists a superstructure where after long translational periods, the overlayer structure reaches the same spot with respect to the surface 'by coincidence'. 
a)

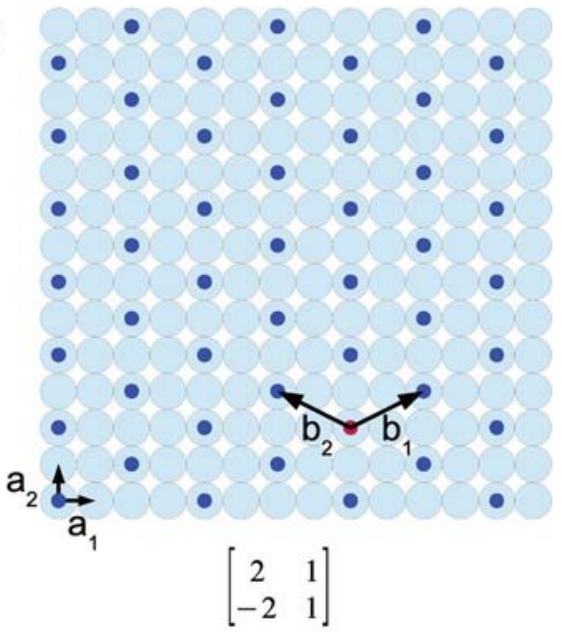

c)

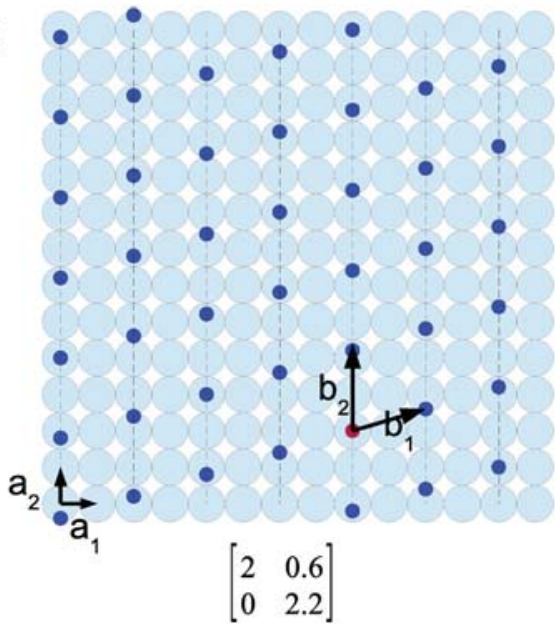

b)

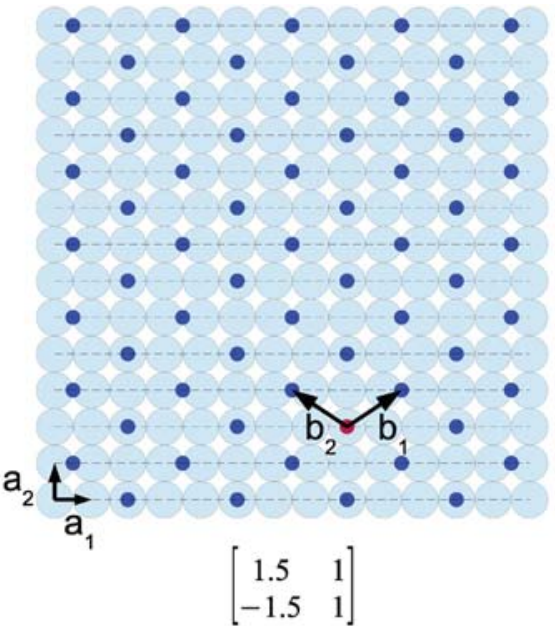

d)

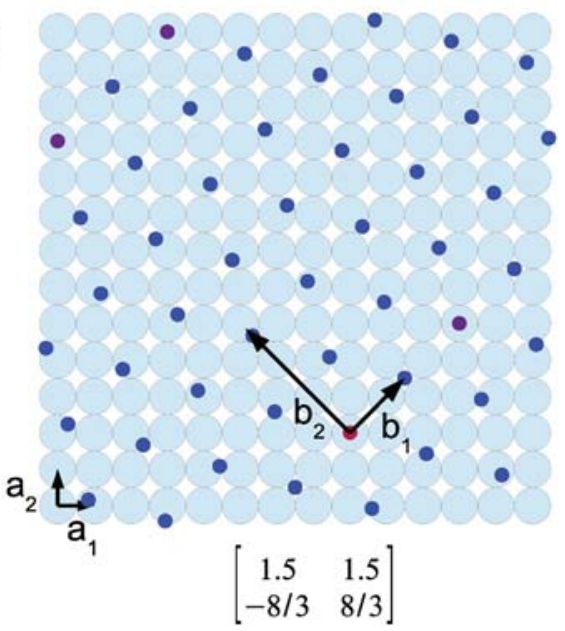

Figure 1. Different types of molecular epitaxy following [59]. (a) Commensurate epitaxy, where every point of the overlayer corresponds to the same point with respect to the substrate lattice. (b) and (c) two different types of point-on-line coincidence epitaxy where every lattice point of the overlayer lies on a primitive substrate line. (d) Coincidence epitaxy. The purple points denote the points where overlayer and substrate match again.

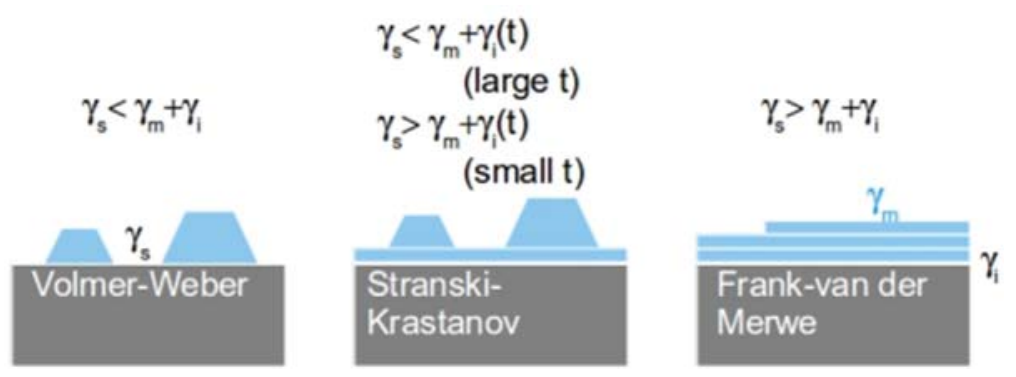

Figure 2. Energetic considerations for molecular growth of molecules on substrates. The different surface and interface energy relations are reviewed.

\subsection{Equilibrium growth and dewetting}

Equilibrium growth can be viewed as resulting from different values of the surface energy, see figure 2. Island growth or Volmer-Weber growth is observed when the surface energy of the substrate $\gamma_{S}$ is smaller than the combined energy of the interface $\gamma_{i}$ and the surface energy of the adsorbate molecule $\gamma_{m}$. The system can gain energy by forming islands and exposing the substrate surface. If on the contrary, the surface energy of the substrate $\gamma_{S}$ is larger than the sum of interface energy $\gamma_{i}$ and the surface energy of the adsorbate molecule $\gamma_{m}$, the system grows in a layer-by-layer growth mode, the Frankvan-der-Merwe growth mode. A mixed growth mode occurs when the interface energy $\gamma_{i}$ depends on the layer thickness. If the thickness of the adsorbate is small, the layer could grow in a layer-by-layer epitaxial fashion involving elastic 
Table 2. In this review, three model aromatic molecules are discussed. Empty fields in this table imply open questions.

\begin{tabular}{llll}
\hline & Pentacene & $\mathrm{C}_{60}$ & PTCDA \\
\hline Geometry of molecule & Flat & Approximately spherical & Flat \\
Rotation of molecule & Absent & Possibly one degree of freedom rotation [32] & Absent \\
Dewetting & observed [43] & Observed [85] & Observed [86] \\
Absorbs at steps & observed [43] & Observed [32] & Observed [33] \\
Charge transfer to surface & Observed [43] & & Absent [47] \\
Electronic hybridization with surface & & Observed [47] & . \\
\hline
\end{tabular}

deformation of the layer. Since the layer is thin, it is energetically not favorable to nucleate dislocations. As its thickness grows, the elastic energy stored in the system becomes larger. In addition, the surface energy change as a function of film thickness. Beyond a critical film thickness, the film continues to grow in islands. Islands grow on a wetting layer in the Stranski-Krastanov growth mode.

Dewetting occurs if the film first grows in a layer growth mode that is not energetically favorable but stabilized by kinetic hindrance. This often occurs if a system having a Volmer-Weber equilibrium growth mode is grown at temperatures lower than necessary for diffusion of adsorbates to the second and subsequent layers. Dewetting can also occur in a situation similar to the one described in the StranskiKrastanov growth mode where the favored film morphology changes with thickness. In contrast to the Stranski-Krastanov growth mode the wetting layer is removed for larger thicknesses as a result of dewetting.

The nature of the dewetting transition is linked to the definition of a disjoining pressure $\Pi$. It is used to describe the difference between the intermolecular interactions and the molecule-surface interactions. If dewetting occurs spontaneously, the situation is described by a negative disjoining pressure, the system gains energy in dewetting. Dewetting can occur due to a long-wave-length instability without overcoming an energy barrier. The process is then limited only by diffusion. In this case it is called spinodal dewetting and has similarities to spinodal decomposition [82]. If an energy barrier is overcome by nucleation and growth of holes, the disjoining pressure $\Pi$ is positive.

$\gamma_{s}(t)=\gamma_{m}+\gamma_{i}(0)+V(t) \quad$ where $\quad \Pi(t)=\frac{\mathrm{d} V(t)}{\mathrm{d} t}$

where the surface energies $\gamma$ are defined in figure 2, $t$ is the thickness of the molecular layer and $V(t)$ is the interaction defining the disjoining pressure.

\subsection{Physisorption and chemisorption on ionic crystalline surfaces}

Often for metal surfaces, there are several phases for absorbed molecules. These occur by physisorption, where the the molecule-surface interaction is governed by van-der-Waals forces, and by chemisorption where the interaction is governed by charge transfer from the molecular levels to the Fermi sea of the metal or vice versa [83]. In the chemisorbed phase, the molecule has an equilibrium position that is closer to the surface compared to the physisorbed phase. Both phases can have an energy barrier between them [84].
On ionic crystalline surfaces, a similar situation occurs concerning the substrate-templated phase compared to the bulk phase [21]. Both phases mainly differ by the epitaxy of the surface and the lateral position of the neighboring molecule with respect to a given molecule. Also here an energy barrier can separate the two phases. Since it is likely that the different lateral ordering also entails a different average distance of the molecule to the surface, these different phases could also be called physisorbed and chemisorbed.

\subsection{Comparison of pentacene, $\mathrm{C}_{60}$ and PTCDA}

In table 2 the different observations reviewed in this article are summarized. Apart from the geometry of the molecules allowing for low-energy excitation of a rotational motion or not, many observations discussed in this review are indeed general for ionic crystalline surfaces.

\section{Ionic crystalline surfaces}

\subsection{Preparation of ionic crystalline surfaces}

Clean ionic crystalline surfaces needed for the deposition of molecules are often prepared by cleaving a single crystal. The freshly cleaved surface is influenced by local charge created during cleavage. In order to remove the charge, the crystals are usually heated in vacuum to speed-up electronic conduction. After removal of the charge, cleaved surfaces typically 50-100 nm distances between steps [33] or even larger, see figure 3. Wedge-shaped structures where the steps are not aligned with high-symmetry crystallographic surface directions result from cleavage and are typical after mild heating.

Atomic resolution is readily obtained on terraces using SFM [23]. The measured contrast arises from atomic-scale electrostatic interactions with a decay length on the order of the lattice constant, see, e.g. [80]. If one thinks of the tip as being covered with ionic material, for example due to contact with the sample, the imaging ion experiences a repulsive short-range force on a surface ion of similar charge and an attractive short-range force on a surface ion of opposite charge [87, 88]. Further insight is gained from simulations [87, 89]. Atomistic simulations often use pairwise potentials, and relax the individual atomic positions under the influence of the calculated forces. Usually shell models are used, where the outer electronic shells are relaxed independently of the atomic positions to represent atomic polarizabilities. First, the force as a function of tip-sample distance is calculated. This force can also be measured experimentally and thus experiment and 


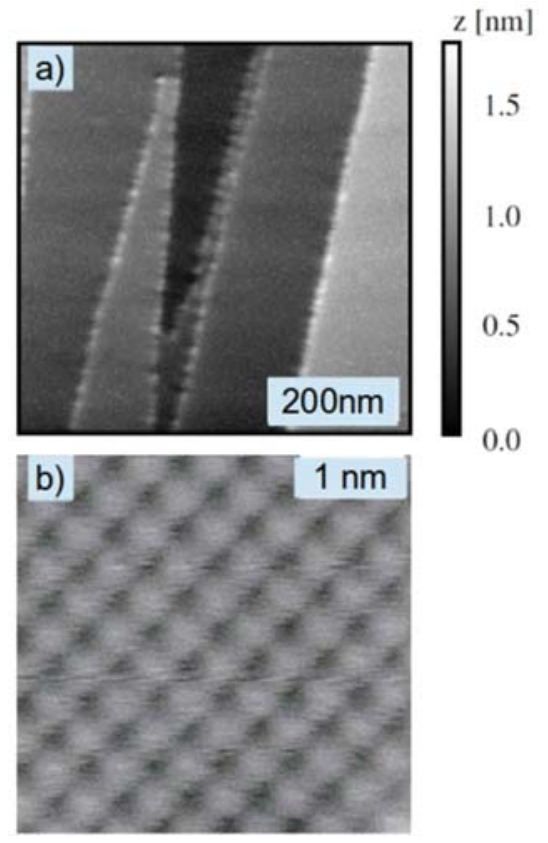

Figure 3. (a) SFM overview over the clean $\mathrm{KBr}(001)$ surface. At the steps, bight objects are observed probably related to the lower coordination of step atoms compared to bulk and surface atoms. Reprinted from [90]. Copyright (2002), with permission from Elsevier. (b) Atomic resolution image of a $\operatorname{KBr}(001)$ surface. Only one type of atoms (either positively or negatively) is imaged as bright due to the attractive forces with ionic material on the tip. The other type of atoms is imaged as a topographic minimum. Reprinted with permission from [87]. Copyright (2004) by the American Physical Society.

theory can be directly compared. For an overview on results on several insulating materials, see $[25,80]$.

A key problem is to determine the charge and chemical nature of the imaging ion. This has first been achieved for $\mathrm{CaF}_{2}$, where the symmetry of the charge distribution is different for positive and negative charge [91]. On $\mathrm{KBr}$, such an asymmetry does not exist, making the problem more complicated. Determining the charge of the imaging ion has first become possible using measurements of the force as a function of tip-sample distance on particular atomic sites and a thorough comparison with atomistic calculations for $\mathrm{KBr}$ and $\mathrm{NaCl}[92,93]$. Later, this technique has been advanced in order to use the three-dimensional force field and to determine even the chemical nature of the imaging ion for $\mathrm{KBr}$ [89]. It has been found that the charge of the imaging ion can be determined from the topographical height differences measured on different ions [94]. Also there have been attempts to determine the charge of the imaging ion from the lateral extension of the imaged ions [95]. However, caution should be taken with this approach, because the lateral extension of the imaging ions depends strongly on the long-range forces and consequently on the sharpness of the tip [96].

\subsection{Steps and charge on ionic crystalline surfaces}

Defects are of particular importance for ionic crystalline surfaces, because at defect positions the surface potential is altered which often means that it is stronger than on the perfect surface. This makes surface defects prone to attract molecules and to form nucleation sites. For an overview of SFM-imaging of defects on insulating surfaces, see [25].

The cleavage process on $\mathrm{NaCl}$-type surfaces typically results in steps running in arbitrary directions forming cleavage wedges. These contain a large amount of kink sites. After mild annealing, these are still present on the surface. After moderate annealing, steps typically run along the [1 00$]$ directions. Perfect steps in these crystallographic are electrically neutral because negative and positive charge equilibrate. At step edges, because of the lower coordination, stronger electrostatic fields occur compared to the surface. At kink sites the coordination is even lower, and the electrostatic fields are even stronger [98] resulting in a strong relaxation of tip and surface atoms upon interaction. This strong interaction between steps and the tip can even lead to reversal of the tip polarity [95]. The potential associated with a step is found to be asymmetric [99].

In the bulk of NaCl-type crystals of the highest available purity charged impurities occur with twofold positive charge such as $\mathrm{Ca}^{2+}[100,101]$. Such positively charged impurities are more frequent than their negatively charged counterparts. This charge is equilibrated at the surface by forming a surface double layer. The negative charge accumulates on the surface and compensates the positive charge in the bulk. This negative charge accumulates at steps in particular trapping negatively charged vacancies at kink sites. Kelvin probe force microscopy images confirm this picture [97], figure 4. In addition to these cation vacancies observed at kink sites, charged color centers are the well-known defects observed in bulk ionic crystals [102].

\section{Methods}

\subsection{Scanning force microscopy methods}

In SFM [24, 103, 104], a sharp tip is brought to close vicinity of a surface such that an interaction occurs, see figure 5 . In the static mode this interaction is measured by observing the bending of a cantilever the tip is attached to with the help of a reflected laser beam. The tip or sample can be precisely positioned with respect to the other by means of a piezo-electric scanner, often a cylinder giving access to the tip-sample interaction as a function of tip-sample distance or as a function of lateral position of the tip. In the dynamic mode, the tip is oscillated [105]. The system of cantilever and tip-sample interaction can be regarded as being composed of two springs operated in series. One of these two springs, the tip-sample interaction, is highly non-linear. The resonance frequency of this system of springs depends on the tip-sample interaction and can be precisely determined experimentally.

The tip is oscillated by an oscillating excitation voltage applied, e.g. to a piezo-electric bar mounted under the cantilever. The resonance frequency of the cantilever as it changes due to the tip-sample interaction can best be determined if the amplitude of the tip oscillation remains constant. A constant-amplitude measurement mode also allows to measure any dissipative tip-sample interactions independently of the conservative forces $[106,107]$. A feed-back loop keeps the 

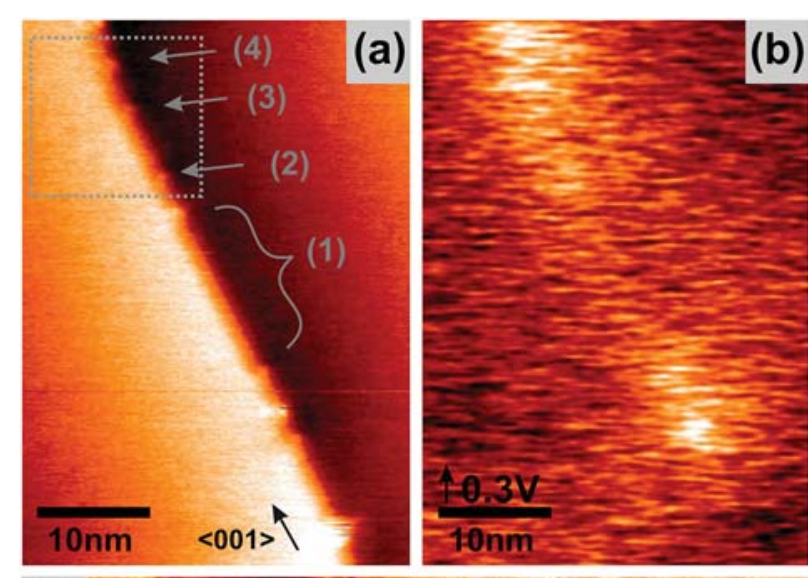

(c)

(4)

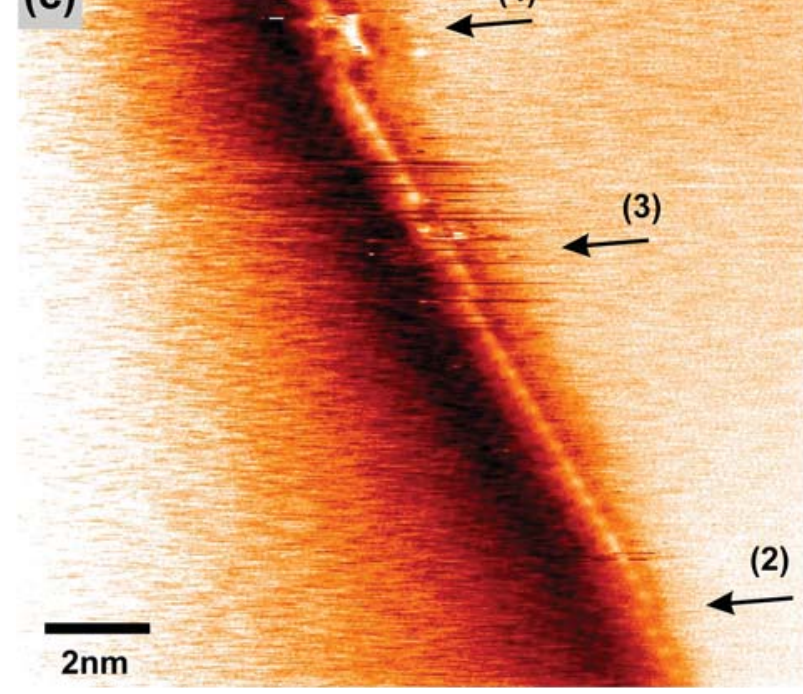

Figure 4. (a) Topography and (b) local contact potential image obtained from SFM measurements on a doped $\mathrm{NaCl}(001)$ surface. The potential scale shows a potential difference of $0.3 \mathrm{~V}$ between the brightest areas and the terrace. Arrows mark cation vacancies at kink sites that lead to a negative surface potential. (c) Constant height image of the area marked with a dotted square in (a). Reprinted with permission from [97]. Copyright (2007) by the American Physical Society.

cantilever oscillation amplitude constant by adjusting the excitation voltage amplitude. The resonance frequency is measured by a phase-locked-loop, containing a feed-back loop that keeps the phase of the oscillation within $2 \pi$ with respect to the phase of the excitation by adjusting the excitation frequency.

The forces measured in SFM are often divided into two groups-long-range and short-range forces. This division does not refer to their precise functional dependence on distance, but to their decay length: forces decaying over a distance similar to $1-10 \mathrm{~nm}$ are called long-range, whereas forces decaying over a distance of less than $1 \mathrm{~nm}$ are called short-range. Long-range forces arise from the van-der-Waals interaction of the body of the tip with the surface and from electrostatic forces due to an applied voltage or due to localized charges [22]. These long-range forces form a background to the high-resolution measurement and are best minimized by using a sharp tip. Short-range forces are chemical bonding forces showing the rich variety known from the quantummechanical treatment of molecules or solids. They strongly

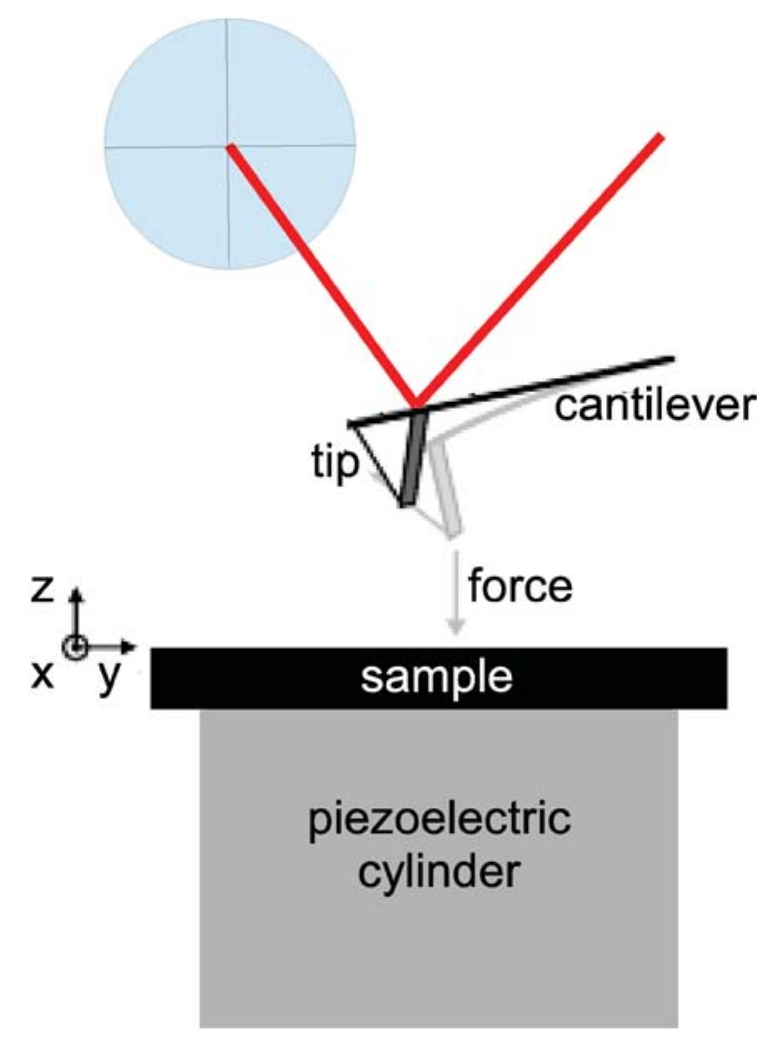

Figure 5. Operation principle of a SFM: the interaction between the tip and the surface causes a bending of a cantilever. This bending is detected by the deviation of a laser beam reflected from the cantilever's surface onto a four-quadrant detector. Dynamic modes rely on oscillating the tip and a measurement of the amplitude or the frequency of this oscillation.

affect the position of the tip apex and surface atoms which relax under their influence.

The frequency shift for a given tip-sample interaction is largest when the oscillation amplitude is on the order of the decay length of the tip-sample interaction to be studied. Since the tip-sample interaction decays on the order of $1 \mathrm{~nm}$ [108], the oscillation amplitude should be of a similar order of magnitude. If imaging is performed with conventional cantilevers, having a force constant of about $40 \mathrm{~N} \mathrm{~m}^{-1}$, this bears the danger of experiencing a snap-to-contact, since the longrange forces arising from the body of the tip add to the shortrange forces and both together can outbalance the restoring force in particular at defect sites. Therefore, often oscillation amplitudes of several $\mathrm{nm}$ are used. On the other hand, tuning forks have been introduced as force sensors, because they have much larger force constants. With these, small oscillation amplitudes below $0.1 \mathrm{~nm}$ can be used [104].

\subsection{Kelvin probe force microscopy}

When a clean Si or metal-coated tip is brought to close vicinity of a sample of a different material, electrons equilibrate the two different chemical potentials. This equilibration will lead to charging of tip and sample surface and to an electric field occurring between the two, see figure 6. This electric field can be measured by SFM and minimized by applying a 
a)

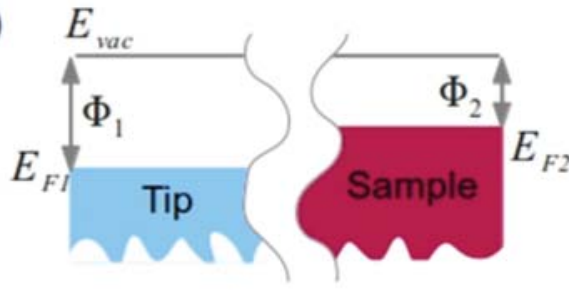

c)

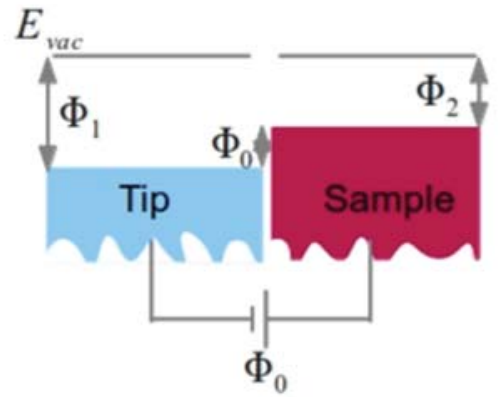

b)

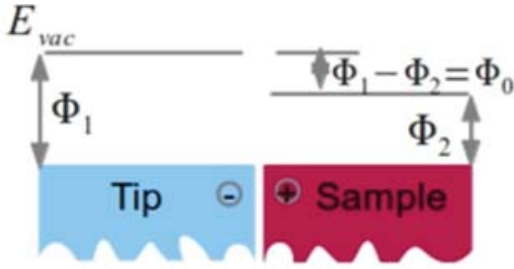

d)

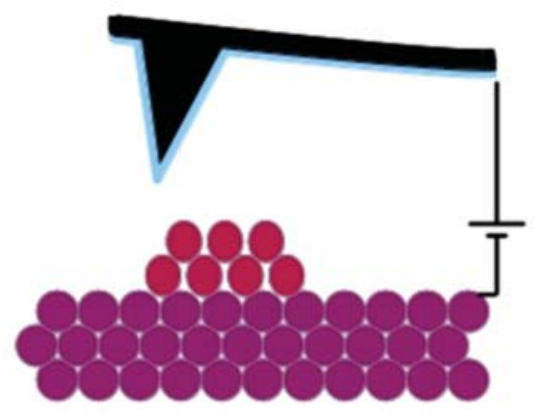

Figure 6. (Principle of operation of Kelvin probe force microscopy. (a) At large distances the tip and the sample have different work functions because they are in general made from different materials. (b) When the tip is approached to the sample electrons can transfer from one to the other and the chemical potential is equilibrated by accumulating charge at the tip and sample surfaces. (c) This charge can be compensated by applying a voltage equal to the contact potential difference to the tip with respect to the sample or vice versa. (d) If the sample is covered by islands of a different material, locally dependent contact potential differences can be measured.

voltage to tip or sample. When this applied voltage equals the difference in work function, the forces are minimized. This method, named after its inventor, Lord Kelvin, can be used to measure the local contact potential difference (LCPD) using SFM [109-111].

For overlayers of a different material, a direct comparison of the two materials' work function is possible, since both of them are measured with respect to the same reference, the tip. Often the view is expressed that the work function of a given material should be constant. This is, however, not always the case. The local contact potential difference depends on the crystallographic orientation of the crystal and can in addition differ for the different parts of a reconstruction as has been observed for $\mathrm{Au}$ [112]. For a molecular overlayer it can also depend on the orientation of the overlayer [42].

For insulators, not only the work function but also localized charges and dipoles can lead to a difference of the local electrostatic field and thus to a change detected in the LCPD measurement [113]. It is found experimentally that the LCPD can also be measured on insulating surfaces, although the meaning of the results obtained has been discussed. The insulating crystal is generally placed on a grounded metallic plate, and its chemical potential is defined with respect to this metallic plate. Since conduction in insulators is not only small but also slow, it is possible that the value of the chemical potential changes slowly at the time scale of the measurement. However, since the movement of the tip is fast compared to the slow variation of the Fermi level, this still allows to measure local contact potential differences precisely. Distance dependent measurements show that the LCPD is constant as a function of tip-sample distance within the experimental noise limit at tip-sample distances above 1.5 $\mathrm{nm}$ [114], see figure 7.

\subsection{Molecular surface dipoles}

The surface potential difference can be used to determine the dipole density at the surface. The surface dipole distribution in a metal is related to its work function $\Phi$ [115].

$$
\Phi=-\mu-\frac{e p}{\varepsilon_{0}}
$$

where $\mu$ is the chemical potential in the metal far from the surface and $p$ is the dipole moment per unit area at the surface of the metal. Such a dipole distribution at the surface exists even for a clean metal [116]. This charge arrangement is altered upon the deposition of additional layers. For molecular overlayers it can be described as the integral over the charge distribution averaged over an area given by the resolution of the tip

$$
p=\frac{1}{A} \int_{A} \mathrm{~d}^{2} x y \int_{0}^{h} z \rho(x, y, z) \mathrm{d} z=\Omega h^{*}
$$

where $h$ is the height of the molecule, $h^{*}$ is the length of the dipole and associated with the effective height of the molecule and $\Omega(x, y, z)$ is the surface charge density given e.g. in Coulomb times m per $\mathrm{nm}^{2}$. As already mentioned, the Kelvin method only provides information about local differences of the work function

$$
\Delta \Phi=\Phi_{2}-\Phi_{1}=-\frac{e}{\varepsilon_{0}}\left(p_{2}-p_{1}\right)
$$

which we can write also as

$$
\Delta U_{\mathrm{CPD}}=-\frac{1}{\varepsilon_{0}}\left(p_{2}-p_{1}\right)=-\frac{1}{\varepsilon_{0}}\left(\Omega_{2} h_{2}^{*}-\Omega_{1} h_{1}^{*}\right) .
$$

Following $[117,118]$, we multiply by the area covered by each molecule and relate the charge density per molecule to the LCPD difference 


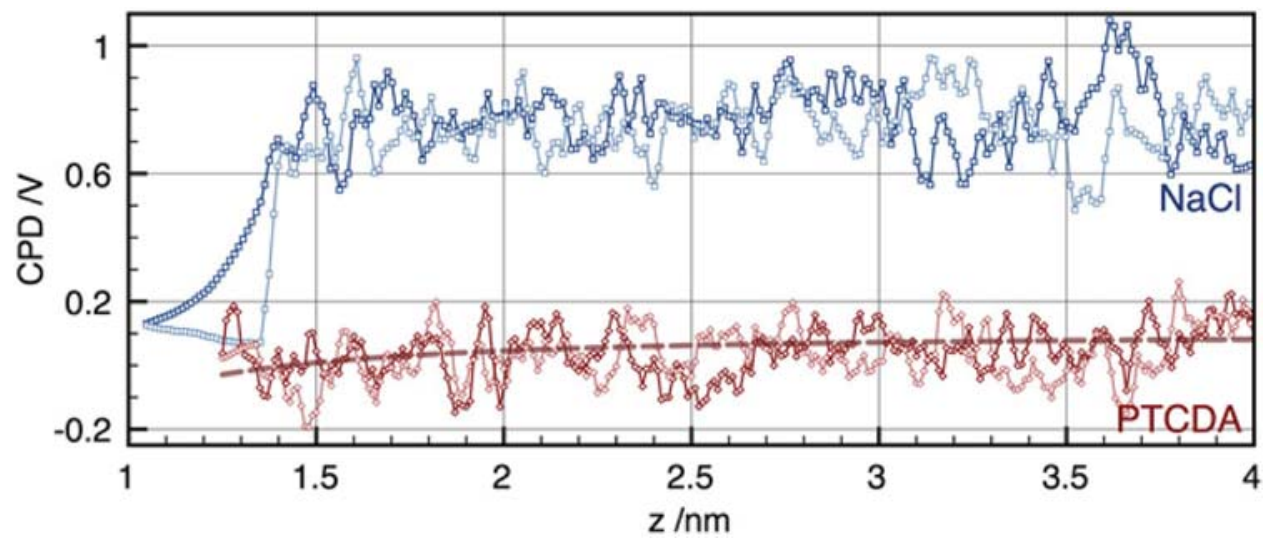

Figure 7. The LCPD measured for PTCDA on $\mathrm{NaCl}(001)$ as a function of tip-sample distance using Kelvin probe force microscopy. Reproduced from [114]. (C) IOP Publishing Ltd. All rights reserved.

$$
\mu_{\mathrm{mol}}=-\varepsilon_{0} \Delta U_{\mathrm{CPD}} A_{\mathrm{mol}}
$$

where $A_{\text {mol }}$ is the area occupied by a molecule. This allows to calculate the charge density per molecule with the measured LCPD and to obtain information on processes such as polarization of the molecule or charge transfer from surface to molecule.

\subsection{Relation of the contact potential difference to the density of states}

The LCPD can also be used to extract the density of states for semiconducting samples [44]. The charge concentration is given by

$$
Q=q \int_{-\infty}^{\infty} g(E) f_{\mathrm{FD}}(E) \mathrm{d} E
$$

where $q$ is the charge of a single carrier, for example $-e$ for an electron, $g(E)$ is the density of states and $f_{\mathrm{FD}}(E)$ is the FermiDirac distribution which is usually relevant only in its tails and approximated as a Boltzmann distribution in semiconductors. We differentiate with respect to the applied voltage

$$
\frac{\mathrm{d} Q}{\mathrm{~d} V}=q \int_{-\infty}^{\infty} g(E) \frac{\mathrm{d} f_{\mathrm{FD}}(E)}{\mathrm{d} V} \mathrm{~d} E .
$$

The Ferm-Dirac distribution is much narrower than the density of states (and $k T \ll E_{\mathrm{F}}$ ), and its derivative can thus be approximated by a delta function.

$$
\frac{\mathrm{d} f_{\mathrm{FD}}(E)}{\mathrm{d} V} \approx \frac{q}{k T} \cdot \delta\left(\frac{E-q V}{k T}\right) .
$$

Since $\delta(x / a)=a \delta(x)$, we obtain

$$
\frac{\mathrm{d} Q}{\mathrm{~d} V}=q^{2} \int_{-\infty}^{\infty} g(E) \delta(E-q V) \mathrm{d} E
$$

and finally

$$
\frac{\mathrm{d} Q}{\mathrm{~d} V}=q^{2} g(E-q V)
$$

which allows to calculate the number of charges from a measurement of the LCPD.

\section{Pentacene}

\subsection{Overview}

Pentacene can be regarded as a piece of monolayer graphite, denoted graphene, i.e. as a nanographene where the edges are saturated with hydrogen, see figure 8 . It is considered a semiconducting molecule and has been largely investigated in the form of a thin film as a candidate for molecular electronics due to its large carrier mobilities [119]. Pentacene has played in important role for the development of atomic resolution on molecules using SFM [36], figure 9. For these studies the molecule was placed on a $\mathrm{NaCl}$ overlayer on $\mathrm{Cu}(111)$ surface, where it assumed a flat-lying configuration. The tip was functionalized by picking up a $\mathrm{CO}$ molecule and the tip-apex was brought to a close distance range using a quartz crystal tuning fork.

Usually pentacene is deposited on the surface by thermal evaporation at $335^{\circ} \mathrm{C}$, see e.g. $[43,120]$. Pentacene is also the product of a single-molecule reaction of precursor molecules on a metal surface triggered in ultra-high vacuum by thermal treatment steps [121]. First, TB2TTA (2,5,9,12-tetrabromoanthra[1,2-b:4,3-b':5,6-b":8,7-b"']tetrathiophene) is deposited by thermal evaporation on a

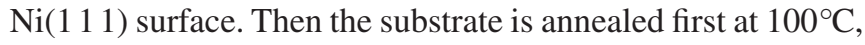
then at $200^{\circ} \mathrm{C}$ such that pentacene is formed on the surface. On the other hand, pentacenequinone molecules deposited on a $\mathrm{Cu}(111)$ surface can be used to grow graphene by thermal treatments [122]. The quinone molecules are deposited onto the heated substrate, then the substrate is heated to intermediate temperatures and finally to $600^{\circ} \mathrm{C}$ to form the graphene layer.

\subsection{Molecular arrangement on the surface}

As many molecules do, pentacene grows in islands on the $\mathrm{KBr}(001)$ surface. Understanding the detailed molecular arrangement in these islands is vital to understand and tune the electronic properties. An important aspect of the molecular arrangement is the question whether molecules are standing upright or lying flat parallel to the surface. Usually, a flat-lying configuration is preferred, when the molecules have 
a)<smiles>c1ccc2cc3cc4cc5ccccc5cc4cc3cc2c1</smiles>

b)

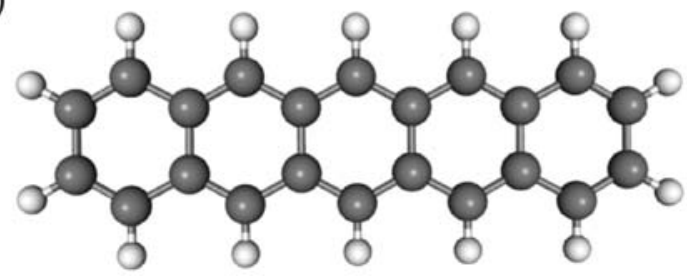

Figure 8. Structure of a pentacene molecule. (a) Chemical notation. Usually the electrons in the benzene rings are regarded as delocalized. (b) Atomic representation.

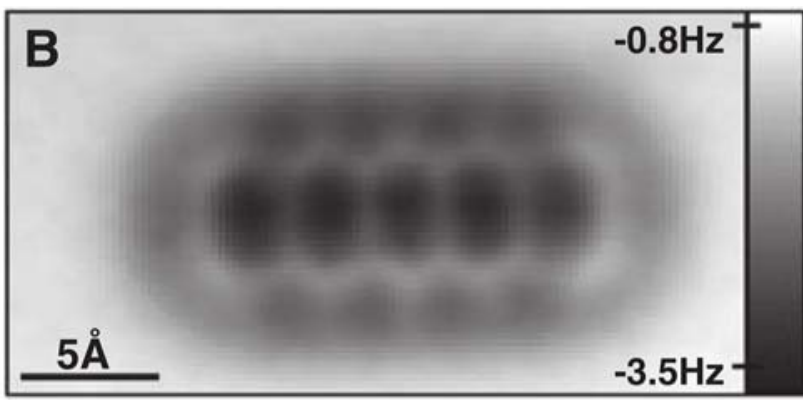

Figure 9. SFM image of pentacene on two atomic layers $\mathrm{NaCl}$ on $\mathrm{Cu}(111)$ imaged with a CO-tip. From [36]. Reprinted with permission from AAAS.

a strong interaction with the substrate and can thus lower their energy in a flat geometry. On the other hand, pentacene has a system of $\pi$-electrons which tend to arrange in parallel to each other due to $\pi$ - $\pi$-stacking. This is easiest to accommodate in an upright standing conformation.

While pentacene grows flat as a single molecule deposited at low temperatures [36], it shows an upright configuration in islands grown at room temperature on bulk $\mathrm{KBr}$ (001) surfaces, see figure 10. As can be seen from the line cut in figure 10(c), the height of the molecular islands can be well explained by an upright standing layer. In such phases, the molecules are not oriented precisely in the perpendicular direction with respect to the surface. It is known that often these molecular layers have a small tilting angle that depends on the details of the molecular arrangement and that differs slightly for different substrates. For pentacene in the bulk phase the tilting angle was found to be $15^{\circ}$ with respect to the sample normal and near zero for the thin film phase [123-126]. These results agree well with $\mathrm{x}$-ray diffraction and electron diffraction studies for pentacene on $\operatorname{KBr}(001)$ and $\mathrm{KCl}(001)$ that also reported nearly upright-standing pentacene islands [127-129].

On $\mathrm{SiO}_{x}$ pentacene grows in a nearly ideal layer-by-layer growth mode in the nearly upright configuration in overall reasonable agreement with our results [130]. This growth
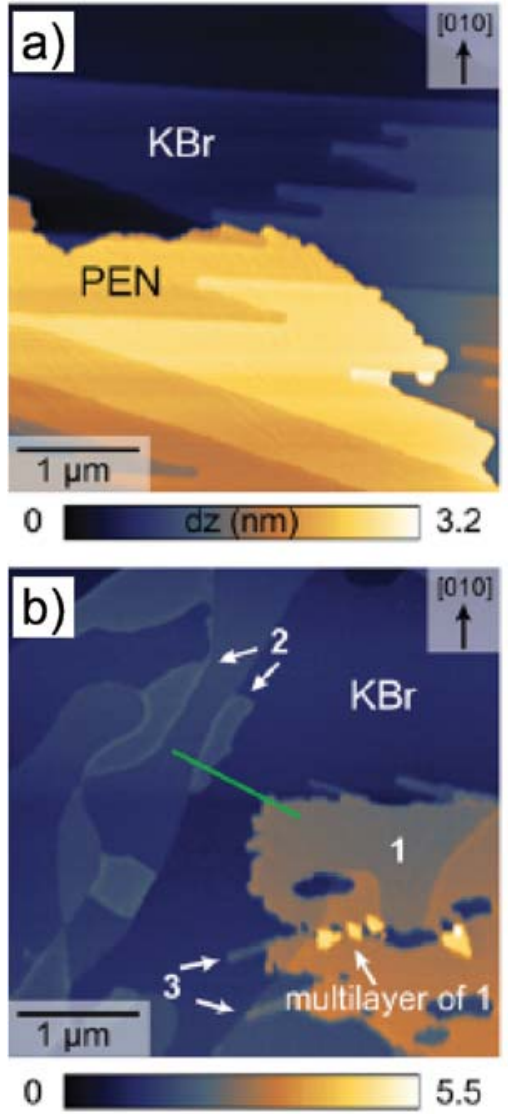

c)

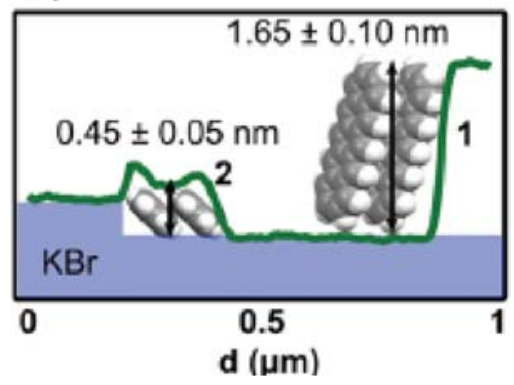

Figure 10. SFM topography images of pentacene islands on the $\mathrm{KBr}(001)$ surface. In (a) one can see typical wedge-shaped structures resulting cleavage of $\operatorname{KBr}(001)$ together with islands of nearly upright-standing molecules. In (b) a second phase of flatlying molecules was observed. This phase is identified from its different measured topographical height compared to the phase of nearly upright-standing molecules as can be seen from the line-cut depicted in green in (b) and in (c). Reprinted with permission from [43]. Copyright (2014) American Chemical Society.

mode is influenced by the presence of a Schwoebel barrier [131]. A critical island size of nucleation of $i^{*}=3$ to 4 was found from a kinetic analysis [132] and $i^{*}=4$ from the scaling behavior of the island size distribution [133]. This critical nucleus is defined from classical nucleation theory, where the free energy is believed to show a maximum at the critical nucleus. If an island grows beyond the critical nucleus it will thus lower its energy by growing further.

$\mathrm{On} \mathrm{Cu}(110)$ pentacene grows lying flat on the surface [134] similar to the growth of the first layer on $\mathrm{Cu}(111)$ [135]. Pentacene induces a charge density wave in $\mathrm{Cu}(110)$ 
that stabilizes the distance between neighboring molecular rows [134] in sharp contrast to its growth on insulators. On $\mathrm{Cu}(221)$ further layers grow in an upright configuration [136]. The first layer grows along step edges, but upon further deposition this order is not transferred to multilayers. On $\mathrm{Au}(1111)$ the first layer also grows lying flat on the surface, while it is standing upright on a polycrystaline Au substrate [137, 138]. The molecules arrange in a striped pattern on the $\mathrm{Au}(110)$ surface [139]. This is in agreement with results on graphite, where also pentacene grows flat on well-ordered substrates, whereas it grows in an upright standing fashion on disordered substrates [140].

On islands grown on $\mathrm{KBr}$ or $\mathrm{KCl}$, we observe an additional substructure, marked by arrows in figure 13(a): line-like structures appear that follow a $45^{\circ}$ or $33^{\circ}$ angle with one of the high-symmetry directions of $\mathrm{KBr}$ and $45^{\circ}, 33^{\circ}$ or $0^{\circ}$ for $\mathrm{KCl}$. This angle is the same within one area. Several of such areas or domains are present within the same molecular island.

In addition to the known phases discussed above one observes a phase of lying tilted molecules (denoted 'phase 2' in the following) unknown for $\mathrm{KBr}$ so far, but known for other surfaces such as $\mathrm{Cu}$ [135]. Such islands are visible through their smaller height compatible with lying molecules. While the first type of islands described above grows over the steps of the underlying substrate such that the substrate steps are visible through the molecular layer, the islands of this new phase remain confined to one terrace. Phase 2 is only observed for samples which show a high density of cleavage steps that are not aligned with the ionic crystalline high-symmetry directions.

A high-resolution image taken at low temperature shows the molecular arrangement in an upright standing island, see figure 11 . The image was affected by thermal drift. In addition, the island apparently had macroscopically changed after the measurement possible due to a strong interaction between tip and molecules during the measurement. In spite of these experimental difficulties we tentatively attributed molecules of a particular arrangement proposed from x-ray diffraction data to the topographic pattern observed, see figure 11. Also a line defect, possibly a domain boundary, has been observed [120]. At the domain boundaries of a pentacene film on $\mathrm{Cu}(111)$, the dissipated energy was found to increase strongly, probably due to the mobility of the molecules near the defect [135]. In figure 12 a structure model obtained from x-ray diffraction studies is shown for comparison [129].

\subsection{Growth and dewetting}

Dewetting is important to understand the morphology of molecular films as they are viewed after deposition. Dewetting changes the morphology of a molecular film during and after growth. It occurs either when a continuous film grows for kinetic reasons, although island growth is energetically more favorable, or when the energy balance of the molecular structures is thickness dependent and favors a film for small coverages whereas for large coverages, island growth is energetically more favorable as in Stranski-Krastanov growth [21]. Images taken several hours to days after deposition allow

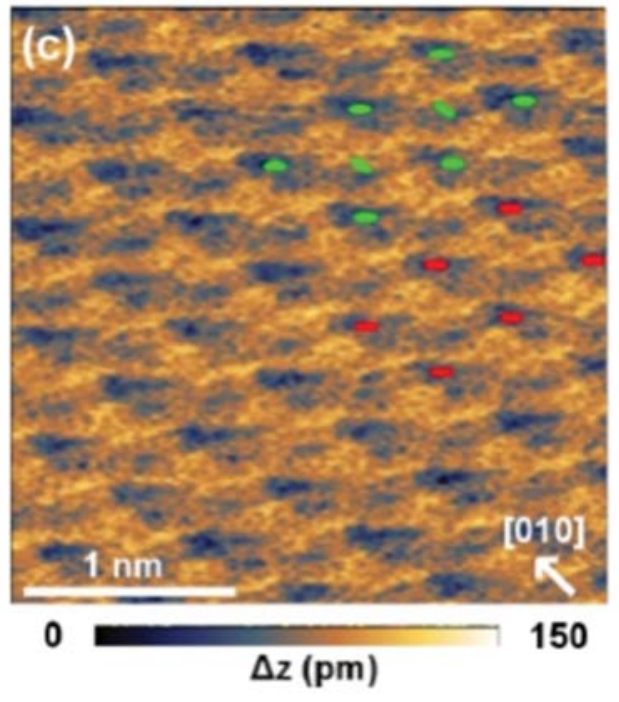

Figure 11. SFM molecular resolution on a pentacene island on $\mathrm{KCl}(001)$ obtained at low temperatures. After taking the image, the island had been modified. Several structures of the molecular layers are proposed due to the large thermal drift and possible modification of the molecular structure through the strong interaction with the tip. Reproduced from [120]. CC BY 3.0.

to study the dewetting process as a function of time, figure 13. The islands shown in this figure appear to have square edges aligned with the (100) and (010) directions of the substrate. A few days after deposition they become irregular and grow holes. In addition, the line-like substructure of the islands becomes more pronounced.

Dewetting often starts at or near defects. One type of defect where we often observe the onset of dewetting are small $\mathrm{KCl}$ islands. Another starting point of dewetting can be the end points of wedge-shaped cleavage structures, see figure 13(c). In addition, it can be found that, as a consequence of dewetting, larger islands with several monolayers height grow at the expense of monolayer islands. Also in figure 14 multilayer islands are shown that grow as a function of time due to dewetting.

In the previous section we introduced phase 1 of upright standing molecules and phase 2 of lying tilted molecules, also denoted by 1 and 2 in figure 10. Islands in phase 2 remain mostly stable as a function of time. Rarely, they are absorbed by phase 1 islands or by growing multilayers. This is mainly observed for islands surrounded by phase 1 islands or near multilayer islands. For isolated phase 2 islands it is also sometimes observed that these islands grow along high-indexed steps.

From x-ray diffraction measurements combined with SFM measurements, a model was generated where, for $\mathrm{SiO}_{x}$ substrates, the center of the islands grow in the orthorhombic phase, covered by a layer grown in the thin-film phase followed by a top ordered in the bulk phase where the tilting angle is slightly different [141]. While we agree that there is a mixture of phases on this surface, this model contradicts our results showing that on $\mathrm{KBr}$ and $\mathrm{KCl}$, by dewetting, islands of one phase grow while islands of another phase become smaller which point to the islands having a homogeneous phase [43]. 

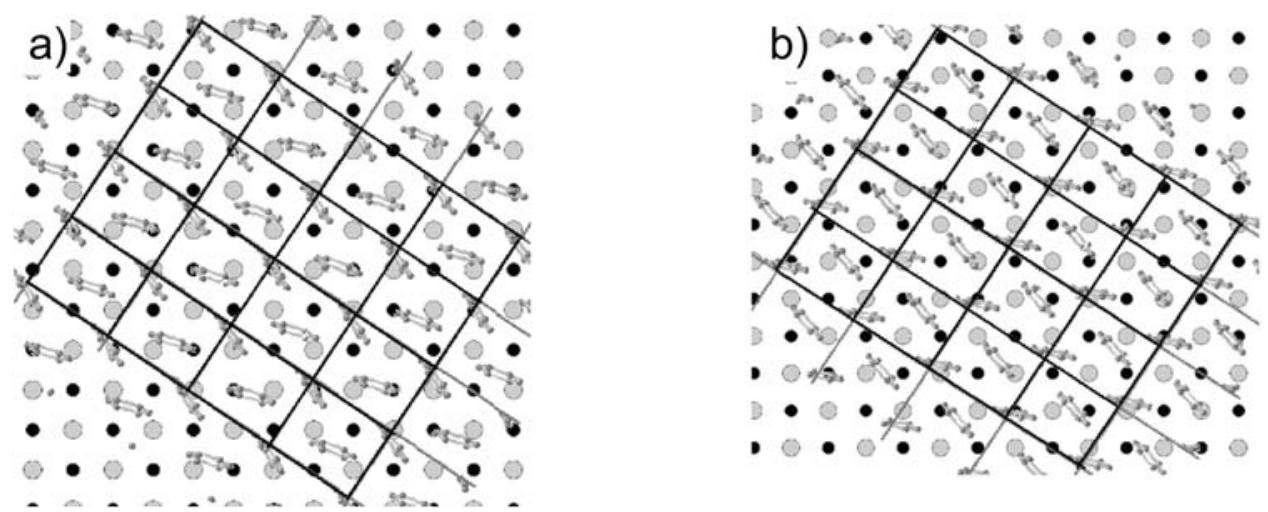

Figure 12. One of the possible phases of the (a) bulk phase and (b) thin film phase of pentacene on $\mathrm{KCl}(001)$ found from $\mathrm{x}$-ray diffraction data. Reprinted from [129], Copyright (2007), with permission from Elsevier.

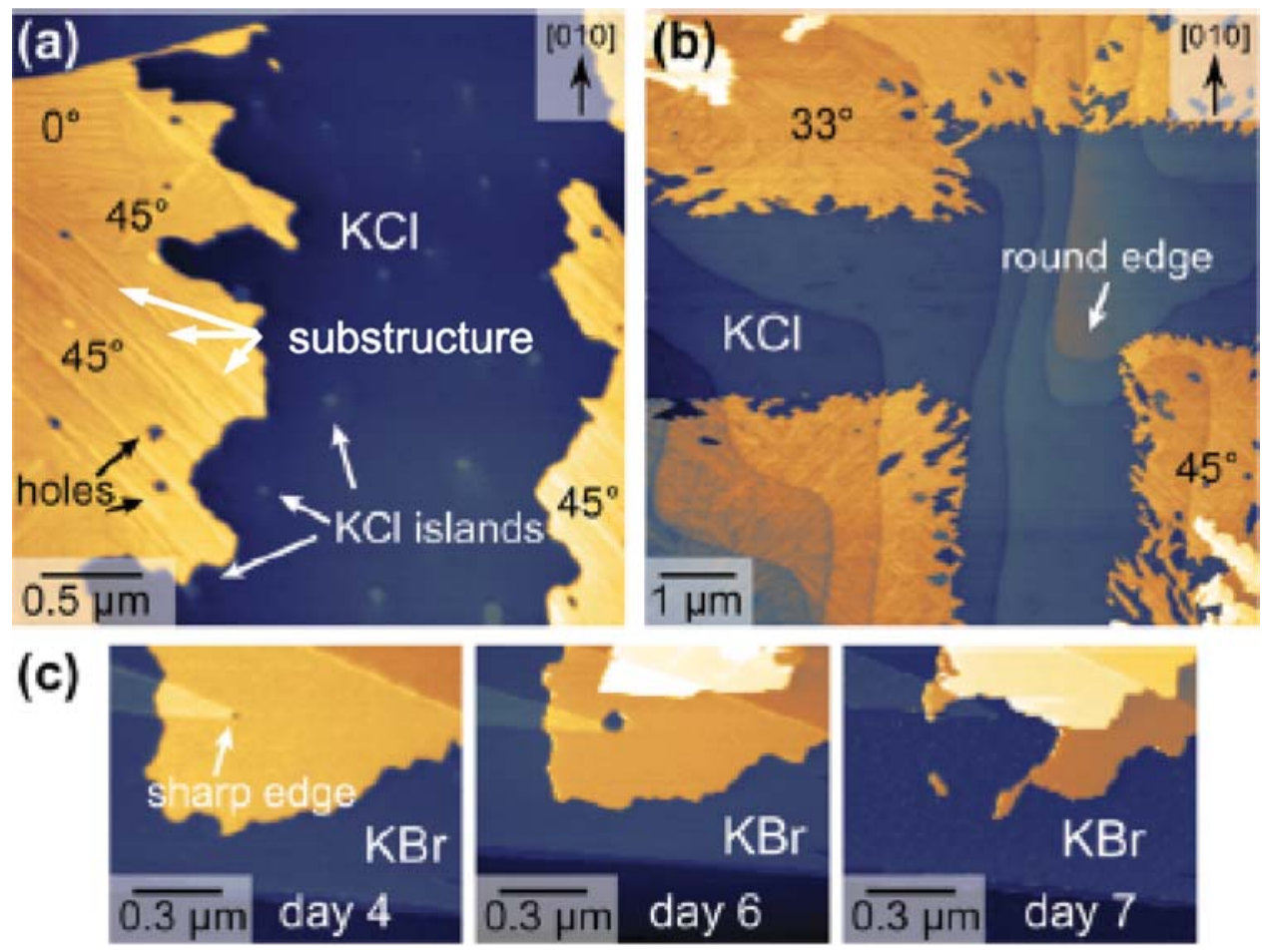

Figure 13. SFM measured dewetting of pentacene on $\mathrm{KCl}(001)$ after (a) $5 \mathrm{~d}$ and —at a different position on the sample-after (b) $8 \mathrm{~d}$. (c) The same position on a $\operatorname{KBr}(001)$ sample at different delays after deposition. Here, one can see the dewetting process starting from a small hole in the film that has formed at the tip of a cleaving wedge of the $\operatorname{KBr}(001)$ surface. Reprinted with permission from [43]. Copyright (2014) American Chemical Society.

\subsection{Electronic properties-local work function}

To evaluate the possible use of pentacene as a molecular electronics material, it is fundamental to understand the electronic properties of the molecular layers. First, we focus on the electronic properties of the ionic crystalline substrate. On average, it must be uncharged. However, already the substrate shows local variations of the work function, i.e. bright lines at step edges. These lines arise from a negative charge accumulated at the step edges [97]. Such a negative charge occurs at the surface in an electric double-layer to equilibrate positive bulk charge due to positively charged bulk impurities $\left(\mathrm{Mg}^{2+}\right)$. At step edges, $\mathrm{K}^{+}$vacancies cause a net negative charge of the step. These bright lines are visible even on top of the molecular layer.
Kelvin-probe force-microscopy images of the moleculecovered surface show that differently oriented phases of pentacene have different work functions, see figure 14. In addition to the bright lines mentioned above, there are black lines in the middle of larger islands. These lines indicate the epitaxial directions of the overlayers in relation to the substrate and could be related to nucleation of the islands.

Analyzing the work function, we compare the polarizabilities of the molecule and the area it occupies in the different phases. We use equation (11) to calculate the dipolar density for the two different phases. We find that this cannot explain the different work functions that we observe. Also in the upright standing phase, the measuring tip is near the positive potential occurring at the ends of the molecules, while the tip is closer to the negative potential of the $\pi$-system when 

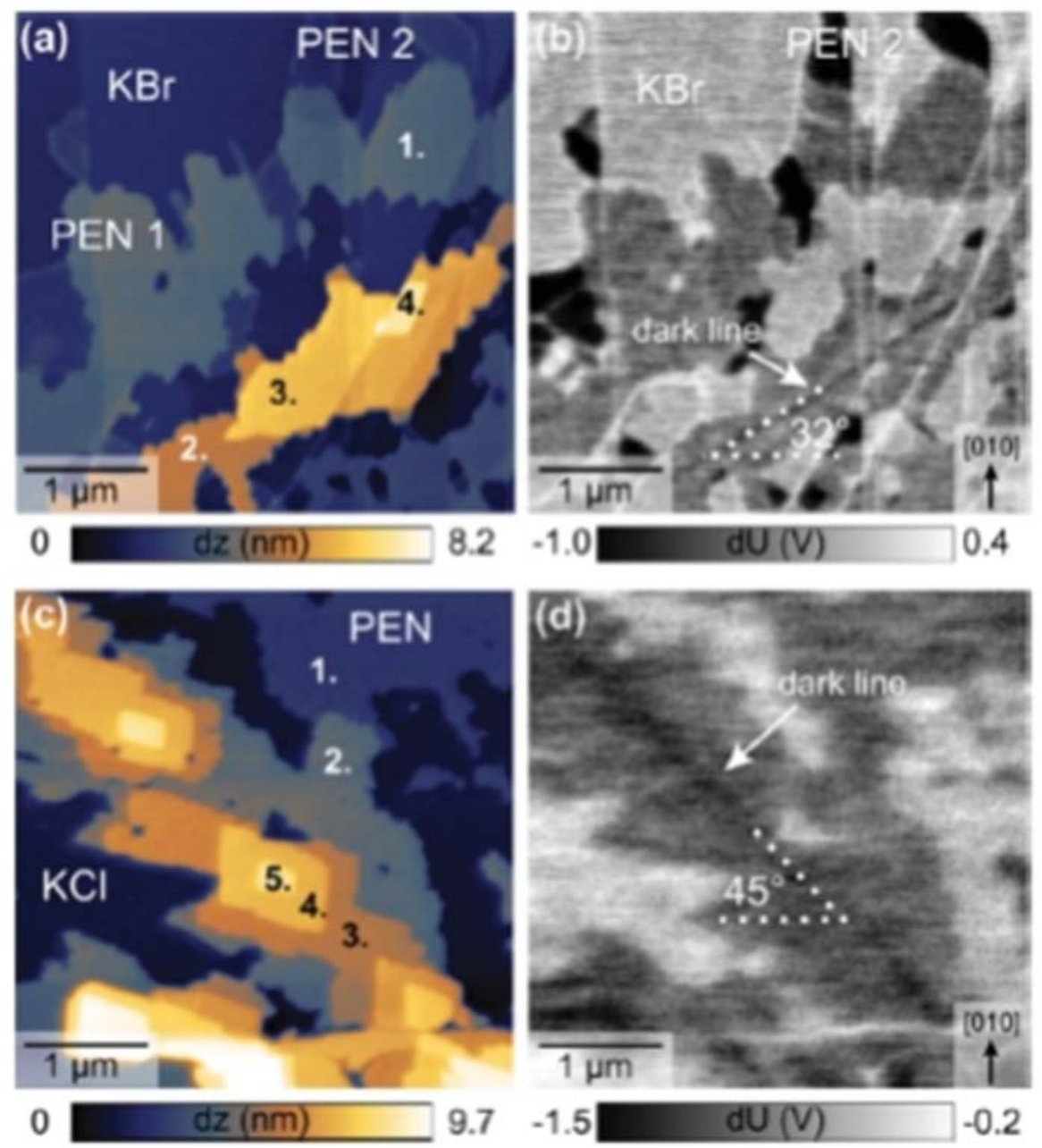

Figure 14. SFM topography ((a) and (c)) and corresponding Kelvin prove force microscopy images ((b) and (d)) of pentacene islands on $\mathrm{KBr}(001)$ after $2 \mathrm{~d}$. One can see in images (a) and (b) that the different phases of upright standing and flat-lying molecules have different work functions. In the center of the islands, a dark line appears. One can also study dewetting in these images. Multilayer islands grow at the expense of monolayer islands. Reprinted with permission from [43]. Copyright (2014) American Chemical Society.

the molecule is flat-lying. This difference between the two phases should lead to the opposite effect on the work function compared to the one we observe. We have estimated a residual electrostatic field above the surface from force-distance measurements and calculated the dipole induced by this field. This field-induced dipole is too small to explain the observed effects. We would expect-due to the strong anisotropy of the molecule's polarizability-to observe a larger LCPD on the phase 1 islands compared to the phase 2 islands, in contrast to our experimental results.

In order to explain the observed contrast, we take charge transfer into account which is in agreement with the low polarization potential for pentacene. If we assume a distance of $0.5 \mathrm{~nm}$ between the charge, 0.012 e would be transferred per pentacene molecule for phase 1 and 0.118 e for phase 2 . Considering that the charge could be distributed over the molecule for phase 2 (the flat lying molecules) but not for phase 1 (the upright standing molecules) the factor of 10 difference seems reasonable.

On $\mathrm{SiO}_{x}$ also a shift of the LCPD has been measured by Kelvin probe force microscopy [142]. Here, it was concluded that the LCPD did not originate from charge transfer but from the dipole induced in the molecule trough the electrostatic field emitted by surface charge on $\mathrm{SiO}_{x}$. On $\mathrm{Cu}(111)$ it has also been observed that the LCPD was larger for the upright standing pentacene layers compared to the flat lying layers [135] similar to our findings for $\mathrm{KBr}$ and $\mathrm{KCl}$ [43]. Also for porphyrin molecules on graphite a dependence of the LCPD on molecular orientation has been found [42]. Charge transfer has also been observed in the case of $\mathrm{C}_{60}$ on hydrogenated diamond [41].

On insulating substrates, charge trapping in pentacene has been investigated [46]. It was found that the charge is strongly localized [45] and trapped mainly in intergrain regions [46]. Charges injected into a single pentacene island remain localized if the island is disordered while it is delocalized over the whole island for ordered islands [48]. Varying the distance of the resistance measurement with respect to an in-plane electrode, the resistance across a grain boundary was found to be large compared to the resistance of a grain for both sexithiophene [16] and pentacene [143]. Similar results were found using two SFM probes also for sexithiophene [15]. Charge trapping can be significantly reduced when additional organic layers are introduced [144]. 


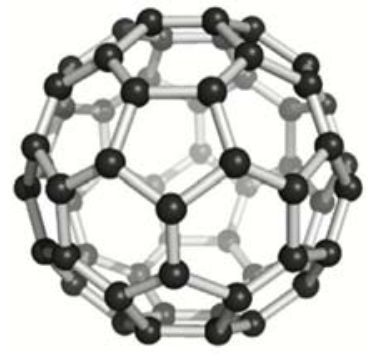

Figure 15. Structure of a $\mathrm{C}_{60}$ molecule.

\section{6. $\mathrm{C}_{60}$}

\subsection{Overview}

On the one hand $\mathrm{C}_{60}$ can be regarded as a prototypical sphere, on the other hand it is a special case of a carbon nanotube with zero length and a rolled-up version of graphene [145]. It consists of an arrangement of pentagons and hexagons known from soccer balls, see figure 15 . This simplicity and prototypical nature has triggered many studies concerning $\mathrm{C}_{60}$. Its interaction potential can be calculated analytically [146]. $\mathrm{C}_{60}$ can easily be evaporated thermally at a temperature of the crucible of about $330{ }^{\circ} \mathrm{C}$. For additional experimental details for sample preparation, see e.g. [32].

A SFM image with resolution of the internal structure of a $\mathrm{C}_{60}$ molecule is shown in figure 16, see [147]. Over the years, imaging $\mathrm{C}_{60}$ has contributed to the development of scanning probe techniques, in particular concerning growth on metal surfaces. Molecular resolution images have first been obtained on metal surfaces using STM where the growth mode was studied [148, 149]. It was found that molecules first absorb at intersections of step edges, then form chains and, at higher coverages, islands. Using SFM in constant force mode, islands grown on silicon and mica substrates were found to have a disordered molecular structure on the top layer [150]. Using an anchoring group attached to $\mathrm{C}_{60}$, a self-assembled monolayer was formed on a $\mathrm{Au}\left(\begin{array}{lll}1 & 1 & 1\end{array}\right)$ substrate and imaged using SFM in constant force mode with molecular resolution [151]. An early study of island growth as a function of temperature on $\mathrm{GeS}(001)$ found a strong dependence on substrate temperature [152]. While at temperatures above $200{ }^{\circ} \mathrm{C}$ only the first layer is stable, at lower substrate temperatures $\left(180^{\circ} \mathrm{C}\right)$ dendritic islands are formed and the higher layers form before the first layer is completed. Growth of $\mathrm{C}_{60}$ on alkali halide crystals has been studied by transmission electron microscopy and by SFM [153, 154]. Hcp- als well as fcc-structured $\mathrm{C}_{60}$ crystals were found. Also for these substrates, nucleation was found to start predominantly at step edges [155]. For an overview on $\mathrm{C}_{60}$ growth on metals, see [4], and for growth on semiconductor surfaces, see [156].

\subsection{Single $\mathrm{C}_{60}$ molecules on surfaces}

The adsorption of single $\mathrm{C}_{60}$ molecules on the $\operatorname{KBr}(001)$ surface is important to understand nucleation of $\mathrm{C}_{60}$-islands on this surface. To study the interaction of single $C_{60}$ molecules
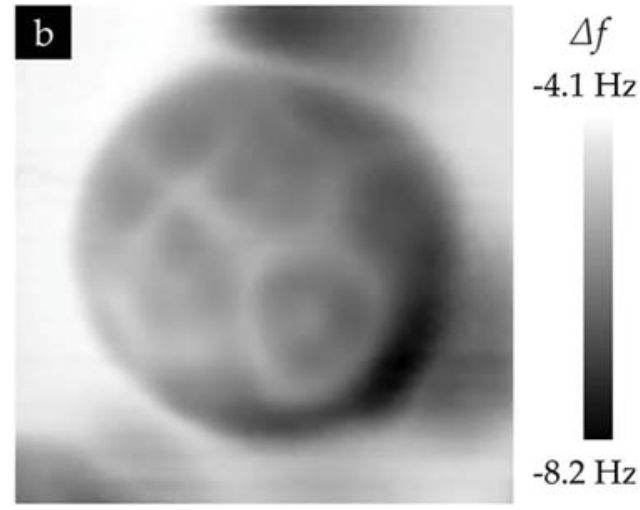

$-8.2 \mathrm{~Hz}$

Figure 16. Frequency shift image obtained in dynamic SFM of a $\mathrm{C}_{60}$ molecule on the anatase $\mathrm{TiO}_{2}(101)$ surface. To obtain the image, the tip was approached by $0.21 \mathrm{~nm}$ closer to the surface compared to topographical imaging. Image size $3 \times 3 \mathrm{~nm}^{2}$. Reprinted with permission from [147]. Copyright (2015) American Chemical Society.

with the $\operatorname{KBr}(001)$ surface in particular at step edges, images across $\mathrm{KBr}$ steps have been taken, see figure 17. As discussed in section 3.2 at step edges, because of the lower coordination, stronger electrostatic fields occur compared to the surface.

For the step edge observed in figure 17, stable imaging conditions are found for most of the step. The step edge is oriented in the [100] direction and therefore, averaged over the length of the step, it is electrically neutral. Similar to the $\mathrm{KBr}$ surface, one type of atom (either the positively or the negatively charged) is imaged as bright, i.e. as a topographic protrusion, while the other type of atom is imaged as black, i.e. as a topographic depression. This contrast can be explained on the clean $\mathrm{KBr}$ surface by adding short-range and long-range interactions between the surface and surface ionic material picked up on the tip [87, 89]. Here, compared to the clean surface an additional complication arises because it is possible that the tip has acquired a molecule from the surface. In view of the atomic resolution achieved it is unlikely that the tip apex consists of a $\mathrm{C}_{60}$ molecule, because then a significant broadening of the surface atoms by convolution with the large tip would be expected. However, more complicated tip shapes including $\mathrm{C}_{60}$ molecules as well as ionic material should be considered.

At the step edge the atoms imaged as protrusions appear somewhat brighter than the atoms of the same type on the terrace. The difference between the step atoms and the terrace atoms is, however, weaker than observed on a $\mathrm{Cu}$ surface covered with a thin layer of ionic material [98]. On the step we observe kinks with even stronger interaction due to the low coordination. In image 17 b) the kinks seem to have the structure expected, whereas for the corner observed in the upper part of the left step, where the upper $\mathrm{KBr}$ step forms a tip, one atom seems to be missing. However, this atom is of the type imaged as dark and as a consequence may not be detected.

We now discuss the streaky features observed at the kink sites and marked with an arrow in figure 17(a) which we ascribe to single $\mathrm{C}_{60}$ molecules. These features have a height of about $1 \mathrm{~nm}$ similar to the diameter of a $\mathrm{C}_{60}$ molecule. The circular shape of the features is clearly distinct from instabilities on 


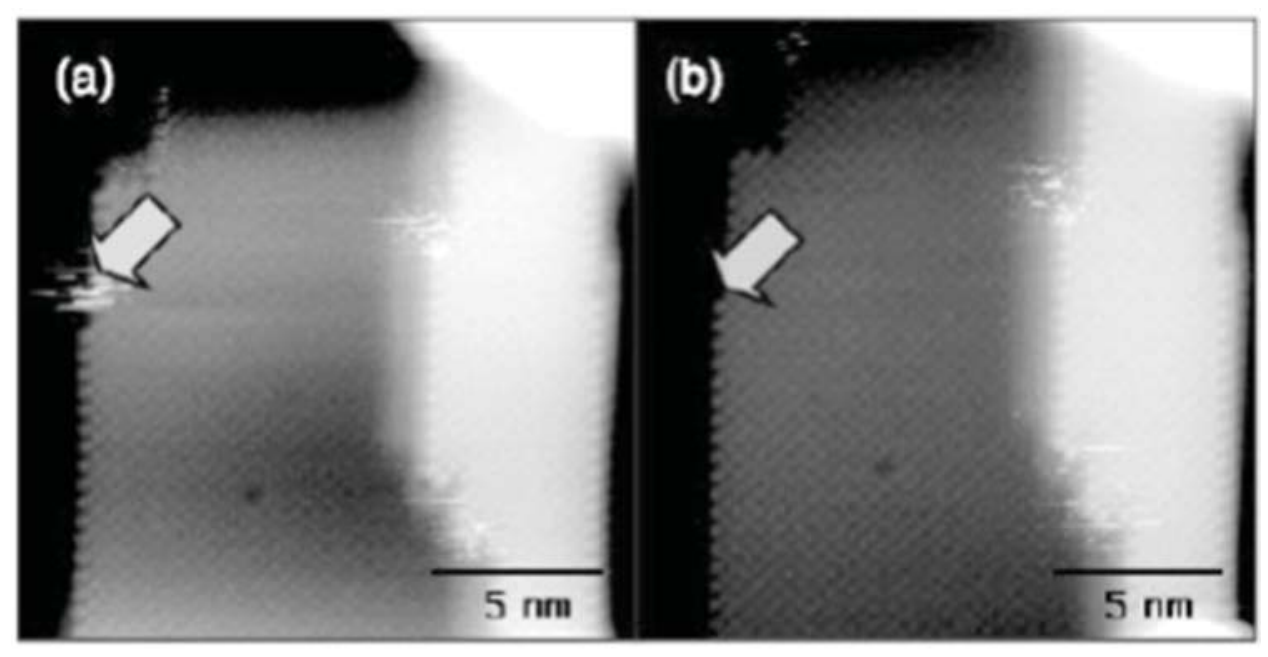

Figure 17. SFM image of single $\mathrm{C}_{60}$ molecule bound to a surface step on the $\mathrm{KBr}(001)$ surface appear as streaky noise in this topographical image. These molecules are bound to kink sites. One is marked with an arrow. Reprinted with permission from [32]. Copyright (2005) by the American Physical Society.

clean surface steps [95]. The lateral extension of the pattern is smaller than double the $\mathrm{KBr}$ bulk lattice constant in radius. From kinetic studies of $\mathrm{C}_{60}$ on $\mathrm{CaF}_{2}$ the critical island size for nucleation has been found to be $i^{*}=1[61]$, this could be the nucleus of a $\mathrm{C}_{60}$ island. This study will be discussed in more detail in the following where the morphology of islands will be discussed. For mobile molecules trapped in nanoscale pits [63] as well as for molecules on metal surfaces in a two dimensional gaseous state [53], similar streaky features have been observed. Similar streaky contrast has been associated in STM images with the motion of single molecules [157], see figure 18 .

The mobility of the molecule occurs on a similar timescale as that of the scan such that for part of some scans, the molecule is located under the tip, while it is elsewhere during the rest of the scans. The location of the $\mathrm{C}_{60}$ molecule can change either by thermal hopping or by the interaction with the scanning tip. In addition to the movement of the molecule that occurs while the molecule remains bound to the kink site, the location of the $\mathrm{C}_{60}$ molecule can change more macroscopically on the timescale of the measurement: the molecule pointed at with the arrow is observed in figure 17(a), but has disappeared only one image frame later in figure 17 (b).

The interaction of $\mathrm{C}_{60}$ with $\mathrm{LiF}$ was computed with special attention to the van-der-Waals (dispersion) contribution and found to be weaker compared to the interaction with semiconductors and graphite [158]. It remains an open question whether $\mathrm{C}_{60}$ molecules prefer to bind to halides or to alkali ions. While one study found that $\mathrm{C}_{60}$ favors an adsorption with a hexagon ring onto a surface $\mathrm{K}^{+}$ion based on density functional theory calculations [159], and experimental study using halides such as $\mathrm{Br}^{-}$on $\mathrm{Au}(111)$ found that bromide adsorbs on top of $\mathrm{C}_{60}$, penetrates and even partly removes $\mathrm{C}_{60}$ from the Au surface [160]. Both studies agree that the $C$ atoms of $C_{60}$ interact more strongly with the halide.

$\mathrm{C}_{60}$ islands are known to grow at step edges, in agreement with single $\mathrm{C}_{60}$ islands as possible nuclei located at step edges. In addition islands grown on terraces are rarely observed. It
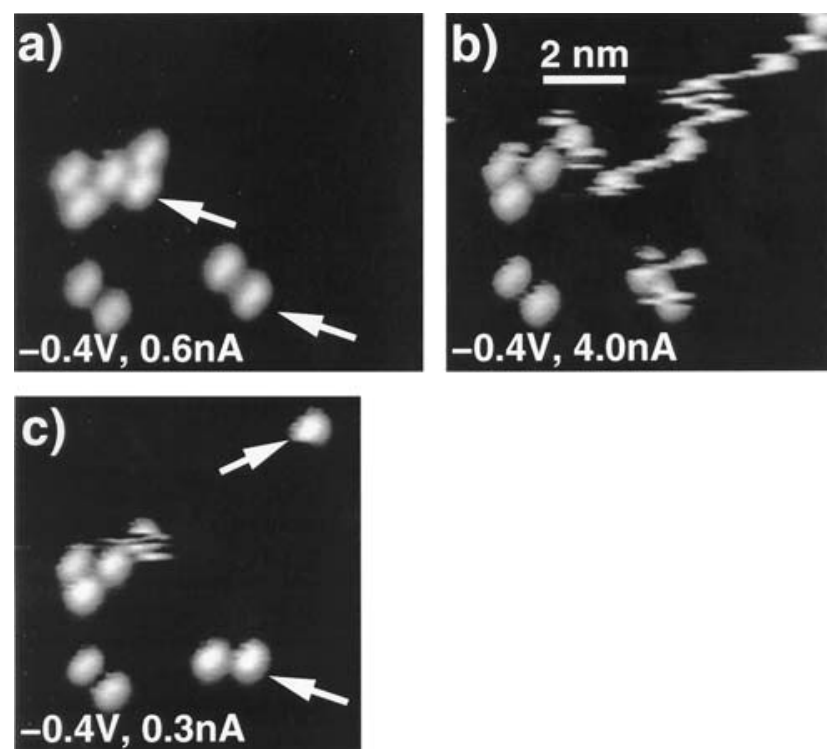

Figure 18. Tip-induced motion as observed during STM imaging of anthracene on $\operatorname{Ag}(110)$ at $50 \mathrm{~K}$. (a) Reference image at large tipsample distance/ small tunneling current. (b) After increasing the tunneling current, streaky contrast is observed. (c) The displacement of the molecules is assessed at small tunneling current. Reprinted from [157], Copyright (1998), with permission from Elsevier.

also remains an open question how $\mathrm{C}_{60}$ islands nucleate on terraces. Defect sites also exist on the free terraces and their density can be increased by sputtering or even by relatively mild treatments such as light electron irradiation [62]. It is well-known for metals that islands nucleate at such defects [161], and a similar situation for $\mathrm{C}_{60}$ nucleation on terraces is likely. Deposition of $\mathrm{C}_{60}$ molecules to the $\mathrm{Si}(111) 7 \times 7$ surface leads to a stable situation where the molecules can be imaged without being moved [162].

Adsorption of molecules to step edges and, in particular, kink sites has also been observed for other molecules such as truxenes [163]. Also for truxenes, streaky noise is observed due to the mobility of the molecules. Here, from a statistic of the length of the stripes, a mean residence time of $200 \mathrm{~ms}$ 

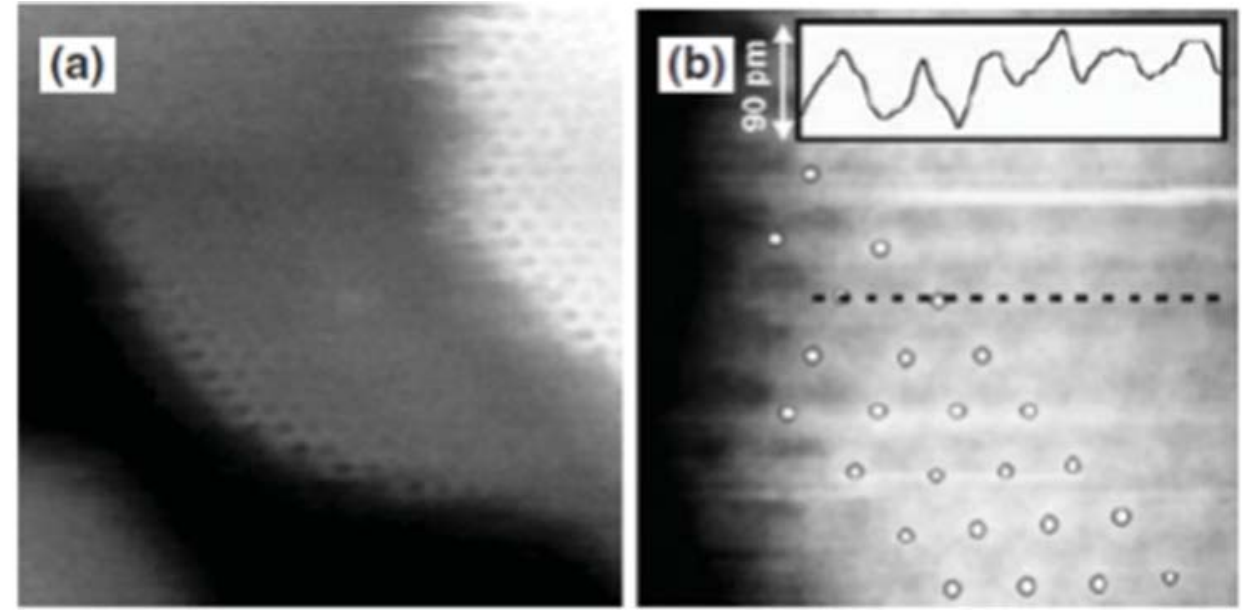

Figure 19. SFM image of an edge of a $\mathrm{C}_{60}$ island on $\mathrm{Au}(111)$. A cut through a molecular resolution image shows that the distances between $\mathrm{C}_{60}$ increase near the edge of the island. We attribute this to a strong interaction of the molecules with the tip. Image size (a) $20 \mathrm{~nm} \times 20 \mathrm{~nm}$, (b) $8 \mathrm{~nm} \times 8 \mathrm{~nm}$. Reproduced from [169]. (C) IOP Publishing Ltd. All rights reserved.

has been found. This could be compared to jumps of $\mathrm{KBr}$ on a $\mathrm{KBr}$ tip, where the mean residence time at low temperatures (about $8 \mathrm{~K}$ ) was determined to be only $60 \mathrm{~ms}$ [107]. It indicates that the interaction energy of the molecules with the $\mathrm{KBr}$ step is larger than that observed in the pure $\mathrm{KBr}$ experiment. This could be explained taking into account that the coordination of the molecule at the step in a confined position between tip and surface is larger compared to a pair of $\mathrm{K}^{+}$and $\mathrm{Br}^{-}$ions. Indeed, it has been observed in calculations that $\mathrm{K}^{+}$and $\mathrm{Br}^{-}$ ions confined between tip and surface can lower their energy by binding to both tip and surface simultaneously [164].

The molecular motion could be influenced by the interaction with the tip. Tip-induced molecular motion has been studied for anthracene molecules on $\operatorname{Ag}(110)$ using STM [157], figure [157]. It has been observed how long-range forces help to assemble molecular species directly beneath the tip leading to contrast inversion [165]. Tip-induced electrostatic long-range forces have also been observed to cause condensation of molecules on metals [53]. From calculations it has been proposed that it is possible to manipulate $\mathrm{C}_{60}$ molecules on the $\mathrm{Si}(001)$ surface using non-contact SFM even though there is no permanent contact between tip and molecule and even though the molecule is covalently bonded to the surface [166]. Furthermore, truxene molecules can even help reshape the surface [167] as has previously been observed for organic molecules on metals [168].

\subsection{Stability of few-molecule islands}

Although there are indications that single $\mathrm{C}_{60}$ molecules are the minimal stable nucleus for $\mathrm{C}_{60}$ island nucleation, still the shape of few-molecule islands varies over time by thermal activation. The shape of such islands is governed by the interplay of temperature and the depth of the energy minimum for each molecule. In addition, the interaction with the SFM tip could influence the shape of the islands. Both effects have been studied in particular at the edge of islands. In figure 19 the edge of a $\mathrm{C}_{60}$ island on the $\mathrm{Au}(111)$ surface has been studied with SFM. Near the edge, the corrugation and the lateral distance between neighboring $\mathrm{C}_{60}$ molecules both increase. In addition there is a change in contrast from close-packed spheres far away from the edge towards an appearance more similar to a honeycomb lattice (an arrangement of apparent holes) near the edge. Also some streaks are observed which are roughly similar to the appearance of single $\mathrm{C}_{60}$ molecules bound at kink sites discussed above. These are indications that the $\mathrm{C}_{60}$ molecules located near the edge are more loosely bound.

A change of contrast as a function of tip-sample distance has also been observed for $\mathrm{C}_{60}$ molecules imaged on rutile $\mathrm{TiO}_{2}(110)$ [170]. This was interpreted with the help of a measurement of the frequency shift as a function of frequency shift setpoint, a quantity that is related to the tip-sample distance, see figure 20. It was found that the frequency shift curves taken at different sites cross in agreement with the data obtained in images. Such a crossing of frequency shift curves had been proposed before for different materials such as $\operatorname{Si}(111) 7 \times 7$ [171] and the $\mathrm{Au}(111)$ herringbone reconstruction [169]. It has been investigated by simulations [172] and for $\mathrm{C}_{60}$ on the $\mathrm{TiO}_{2}$ surface [170].

Molecular resolution has also been obtained at a $\mathrm{C}_{60}$ step edge on the $\operatorname{KBr}(001)$ surface at low temperatures [173]. In this case, there was no indication of mobility of the molecules. In order to decide whether the temperature or the interaction of the tip had the dominant role in inducing mobility at the step edge, we can compare the interaction of the tip with the surface used in both experiments. For the experiment on the Au surface [169], from the parameters given (frequency shift $\Delta f=-40 \mathrm{~Hz}$, resonance frequency $f_{0}=150 \mathrm{kHz}$, longitudinal spring constant $c_{L}=50 \mathrm{~N} \mathrm{~m}^{-1}$ and oscillation amplitude $A=6 \mathrm{~nm}$ ), we calculate the normalized interaction $[174,175]$

$$
\gamma=c_{L} \frac{\Delta f}{f_{0}} A^{3 / 2}
$$

to be $6.2 \cdot 10^{-15} \mathrm{~N} \mathrm{~m}^{1 / 2}$ whereas we find $\gamma=2.5 \cdot 10^{-16}$ $\mathrm{N} \mathrm{m}^{1 / 2}$ for the experiment on $\operatorname{KBr}(001)$ from the parameters $\left(\Delta f=-2 \mathrm{~Hz}, f_{0}=26550 \mathrm{~Hz}, c_{L}=1800 \mathrm{~N} \mathrm{~m}^{-1}\right.$ and $A=0.15 \mathrm{~nm})$ used for the tuning fork in [173]. As a 


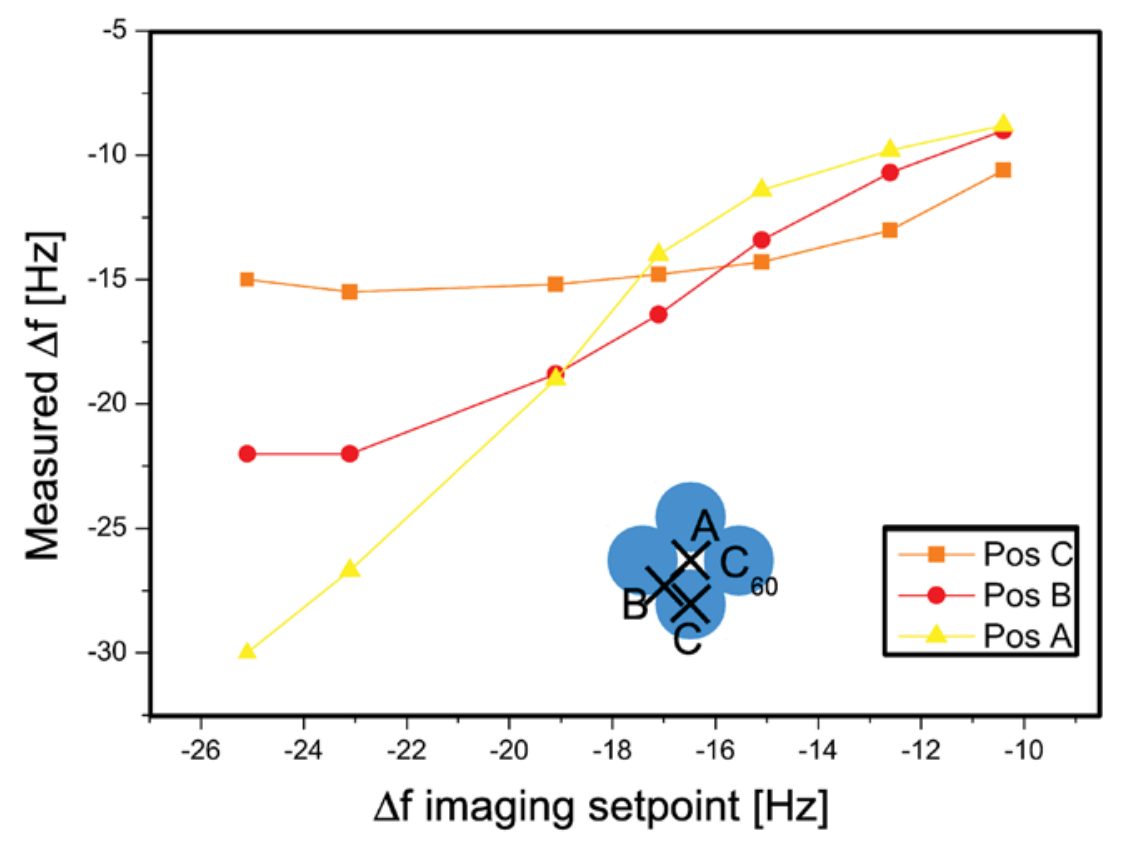

Figure 20. The frequency shift was measured during imaging at different frequency shift imaging setpoints $\Delta f$ at the position in the center between four $\mathrm{C}_{60}$ molecules (position $\mathrm{A}$ ), between two neighboring $\mathrm{C}_{60}$ molecules (position $\mathrm{B}$ ) and on top of a $\mathrm{C}_{60}$ molecule on the rutile $\mathrm{TiO}_{2}(110)$ surface using dynamic SFM. Since the frequency shift imaging setpoint is a function of tip-sample distance, the data is related to a frequency-distance measurement and confirms contrast inversion. Adapted from [170]. @ IOP Publishing Ltd. All rights reserved.

conclusion, in the experiment on $\operatorname{KBr}(001)$ [173] not only the temperature, but also the tip-sample interaction was much smaller compared to the experiment on $\mathrm{Au}(1111)$ [169], and thus it is not simple to detect which of the two was decisive here.

In figure 21 a $\mathrm{C}_{60}$ cluster imaged with non-contact SFM on the $\mathrm{Au}(1111)$ surface is shown as described in [169]. The orientation of the $\mathrm{C}_{60}$ molecules with respect to the $\mathrm{Au}$ substrate cannot be determined from the image. Possibly the molecule is rotating in the room temperature measurement. During consecutive scans molecules are removed from the cluster and therefore the number of molecules composing the cluster constantly decreases. However, no asymmetric shapes or defects are observed. Even if just one molecule is removed from a cluster of 19 molecules, a rearrangement of the cluster leads to a new close-packed structure with small surface energy. The molecules rearrange in a close-packed fashion due to the influence of temperature and/or the interaction with the scanning tip. More and more $\mathrm{C}_{60}$ molecules are removed from the 19 molecule cluster until the final cluster contains only 7 molecules. This is in agreement with a simulation based on the potential derived by Girifalco [146], where the clusters composed of 19 and 7 molecules, as well as the ones formed from 37 and 61 molecules, are found to be more stable structures of $\mathrm{C}_{60}$ on graphite and could be called magic clusters [177].

From mass spectrometry, from the periodic table of elements as well as from nuclear physics, e.g. it is well-known that an arrangement of objects can be particularly stable if closed shells are formed or a quantum-mechanically particularly stable state is reached. It is well-known that the raregases are chemically particularly inert due to closed electronic shell formation. For clusters found in mass-spectrometry measurements, also stable electronic shells can influence the stability of clusters of a particular size, called magic clusters. In addition to electronic closed shells, also the atomic arrangement can form closed shells, also leading to particularly stable clusters. A similar phenomenon has been observed for stable clusters of molecules on surfaces. On the $\operatorname{Ag}(111)$ surface, 1-nitronaphthalene leads to a chiral arrangement due to the structure of the molecule [176]. Clusters containing $10 \mathrm{~mol}-$ ecules (decamers) are found to be much more stable compared to clusters containing 11 molecules (undecamers), see figure 22. For borazines on $\mathrm{Cu}(111)$, regular heptamers appear to be most stable [178]. In addition, clusters composed of 13 molecules appear in richer abundance compared to other clusters.

\subsection{Rotation of single molecules in islands}

It has been debated whether $\mathrm{C}_{60}$ molecules within islands at surfaces can rotate by thermal activation at room temperature. Through the high symmetry of the molecule, the rotational positions of the molecule are roughly equivalent with only a small deviation due to the atomic arrangement. To access all angles of a full rotation two angles are necessary. The question arises whether on $\mathrm{KBr}(001)$ both of these degrees of freedom are thermally activated or just one of them.

When studying $\mathrm{C}_{60}$ molecules at room temperature, we have observed two different heights, see figure 23 . The observation of $\mathrm{C}_{60}$ molecules with different heights implies that one of the two rotational degrees of freedom is fixed such that the $\mathrm{C}_{60}$ molecule is oriented with a hexagon or a pentagon facing the substrate. The other rotational degree of freedom could still be thermally activated and the molecule could rotate around its central axis. 

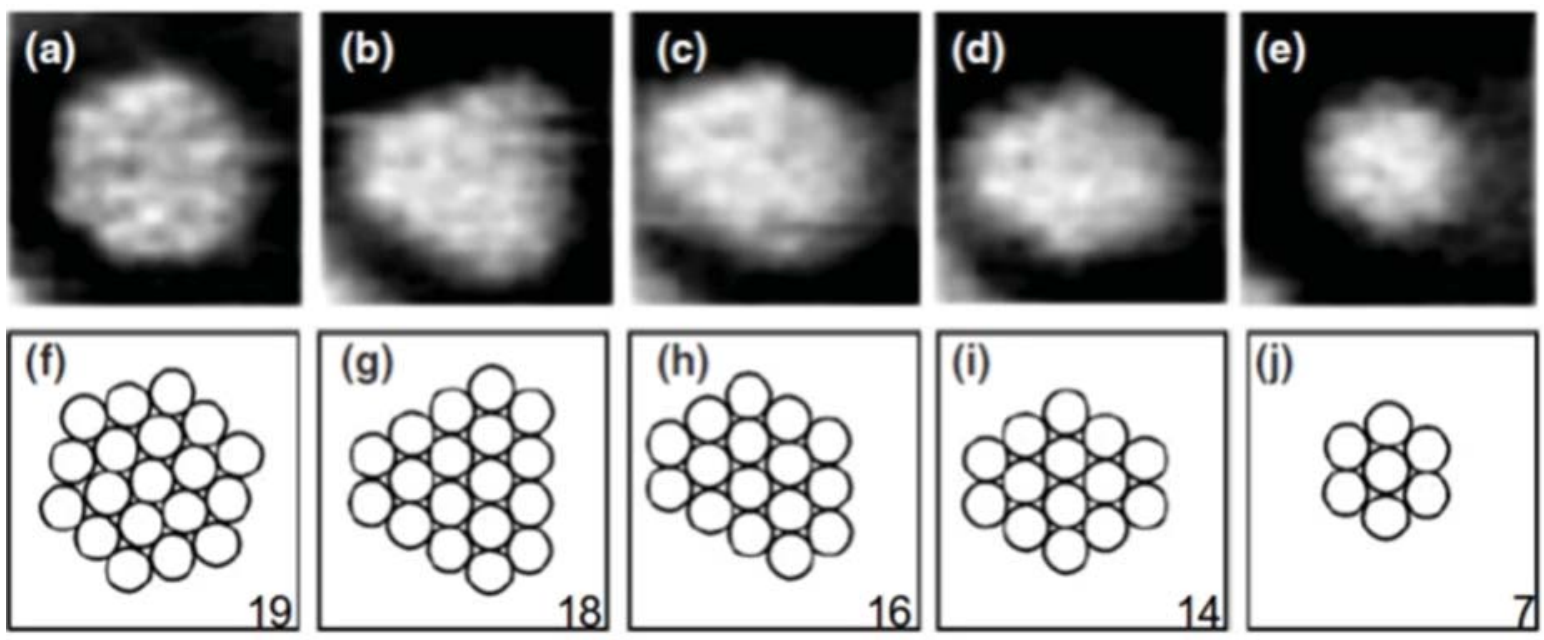

Figure 21. (a)-(e) SFM images of clusters of $\mathrm{C}_{60}$ molecules observed on the $\mathrm{Au}(111)$ surface. (f)-(j) Below each image, a model of the structural arrangement of each cluster is depicted. The images have been taken consecutively at the same lateral position. During scanning more and more $\mathrm{C}_{60}$ have been removed from the cluster. The number of molecules is depicted in each model arrangement. Size of each image: $6 \mathrm{~nm} \times 6 \mathrm{~nm}$. Reproduced from [169]. (C) IOP Publishing Ltd. All rights reserved.
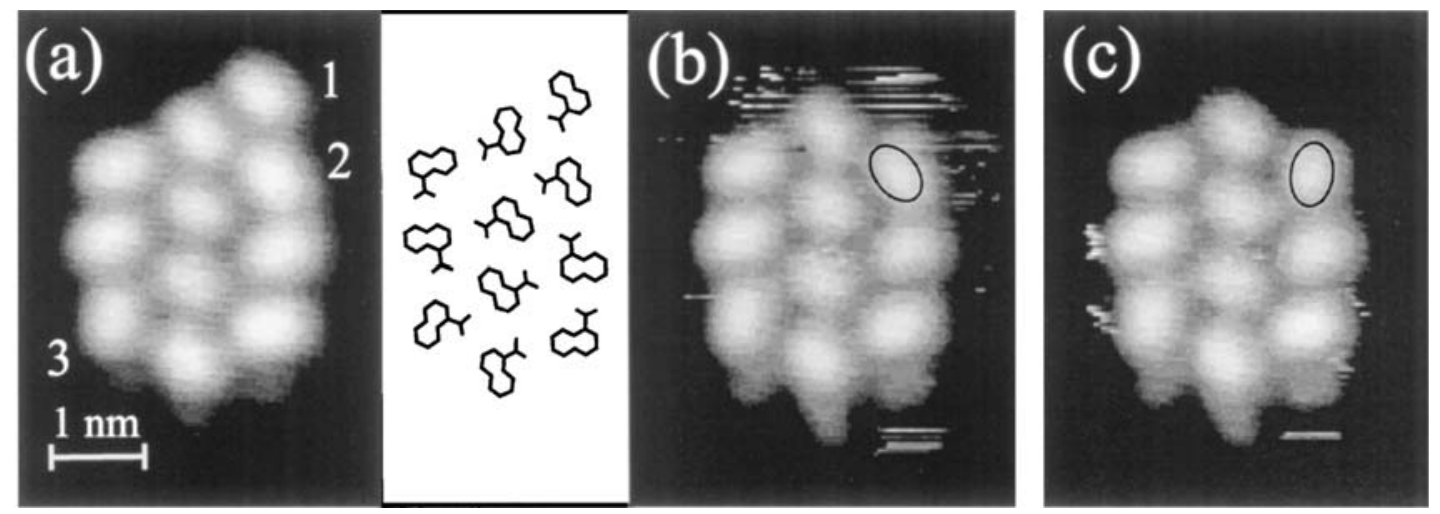

Figure 22. STM images of 1-nitronaphthalene on reconstructed $\mathrm{Ag}(111)$. (a) Image of a cluster containing 11 molecules observed at $50 \mathrm{~K}$ and proposed structure. (b) One molecule is removed by scanning with a larger tunneling current. (c) Resulting cluster composed of 10 molecules. Note that the molecule denoted by ' 2 ' has been rotated by ca $50^{\circ}$ clockwise. Reprinted with permission from [176]. Copyright (1999) by the American Physical Society.

Such a different height of molecules has also been known for $\mathrm{C}_{60}$ on metals $[180,181]$. On metals, this contrast can be related to the interaction of the $\mathrm{C}_{60}$ overlayers with the metal, causing charge transfer and adsorbate-induced surface reconstructions. It has also been debated whether height differences of differently oriented molecules could lead to such a contrast on metals. The molecular structure of the topmost atoms on differently oriented molecules have been resolved by low temperature STM showing that at low temperatures the molecular rotation is blocked [179, 182, 183], see figure 24. Similar effects have also been observed using SFM on insulating surfaces [184]. Also from SFM imaging contrast obtained on the $\operatorname{Si}(111) 7 \times 7$ surface, the precise orientation of a $\mathrm{C}_{60}$ molecule located at the apex of the tip can be determined [185].

The rotation of $\mathrm{Cu}$-phtalocyanine molecules on a $\mathrm{C}_{60}$-island substrate has also been studied as a function of temperature $[186,187]$. The molecular rotation is blocked at low temperatures, whereas the molecule can hop between six different orientations at room temperature. At room temperature only a temporal average of the molecular motion is observed.

\subsection{Epitaxy}

Determining the epitaxial relationship between the $\mathrm{C}_{60}$ islands and the ionic crystalline substrates is difficult since the interaction between the molecules and many substrates is weak. For $\mathrm{C}_{60}$ on $\operatorname{KBr}(001)$, the distance between islands is often several hundred to thousand $\mathrm{nm}$, and often given by the distance of the cleavage steps on the $\mathrm{KBr}$ surface rather than by the molecule-surface interaction. The density of islands on the bare substrate may be governed by the defect density rather than the molecule-surface interaction. As a consequence the island shapes are strongly influenced by kinetic effects and it could be misleading to try obtaining information about the epitaxial relationship between molecules and surface from the island shapes.

Information on the energetically most favorable molecular arrangement is obtained by observing the high-temperature structure of an island, see figure 25 . As expected from the close-packed arrangement, the molecular islands assume a hexagonal shape. The island edges are arranged parallel to $\mathrm{KBr}$ step edges, which run in the (100) direction and 


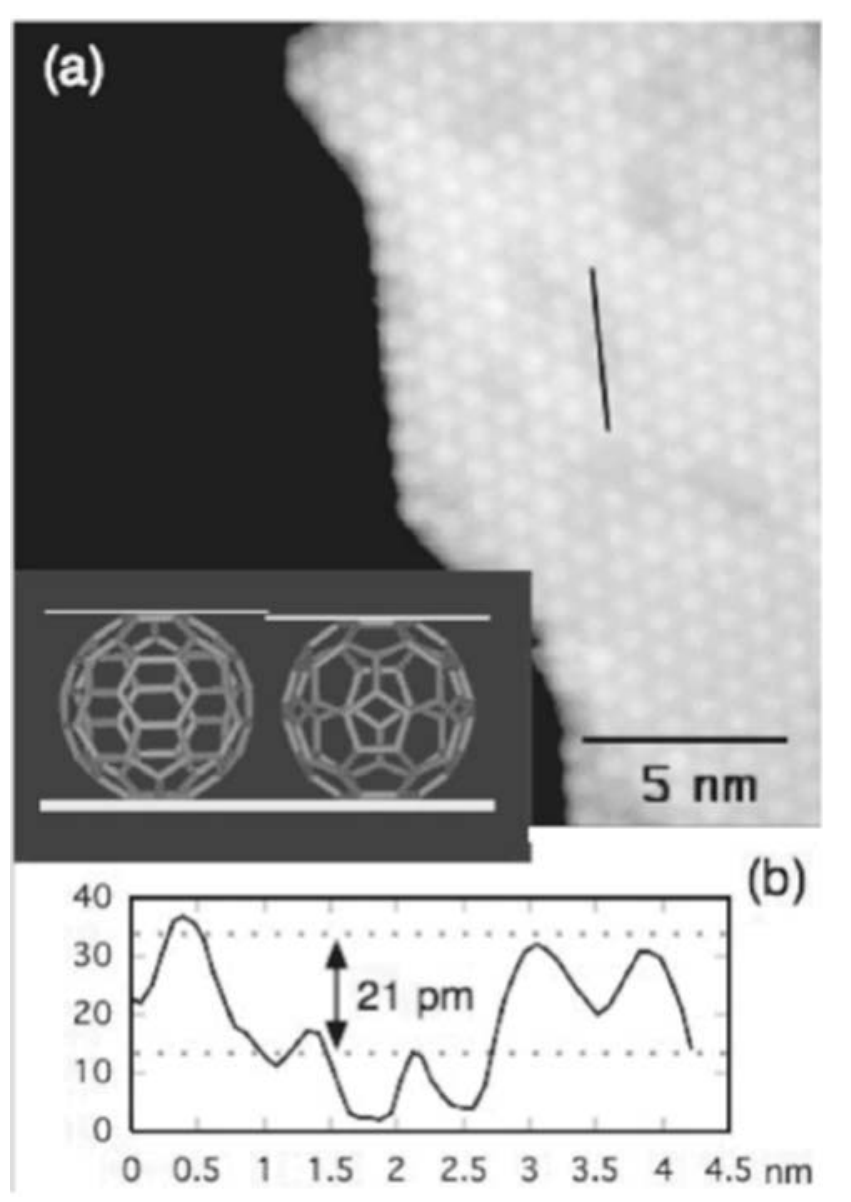

Figure 23. In SFM images of islands of $\mathrm{C}_{60}$ molecules on $\mathrm{KBr}(001)$ topographically higher and lower molecules are observed as can be seen in the line-cut marked in black in (a) and in (b). The height difference corresponds to a different orientation of the $\mathrm{C}_{60}$ molecule, i.e. sitting on the pentagon versus sitting on the hexagon. Reprinted with permission from [32]. Copyright (2005) by the American Physical Society.

equivalent. This could show that the templating effect from the substrate steps is stronger than epitaxial preferences.

From imaging an island and the substrate in the same image with atomic resolution on the substrate and molecular resolution on the molecular island, residual image imperfections related to the piezo-electric motion can be corrected and the epitaxial relationship between the island and the substrate can be determined. A possible structure, derived from images similar to the one shown in figure 26 in that way, is shown at the bottom of the figure. It is a coincident structure of the type depicted in figure $1(\mathrm{~d})$.

An additional structure has been observed in a further study [188]. In this study, additionally to the method described above, a moire pattern has been observed and was used to derive the epitaxial relationship. Both structures agreed with each other within the experimental error but differed from the one described in figure 26. As a consequence, we believe that the molecules can grow in a variety of epitaxial structures on the $\operatorname{KBr}(001)$ surface since the energetic differences between the different structures are small. Also there seems to be no clear relation between the epitaxial relationship and the morphology of the islands. Similar epitaxial a)

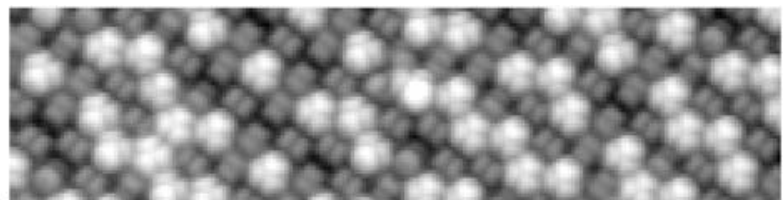

)

b)

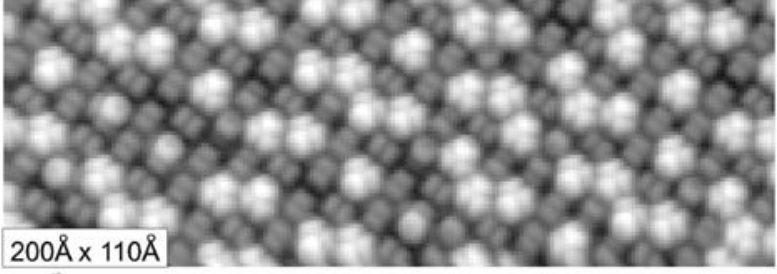

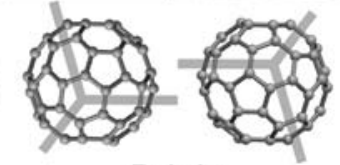

Bright

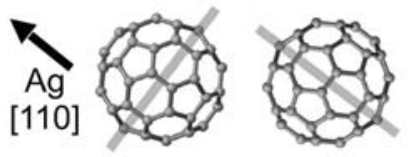

$\operatorname{Dim}$
Figure 24. (a) STM image of $\mathrm{C}_{60}$ on $\mathrm{Ag}(001)$. Bright and dim molecules are observed. (b) Schematic diagram of the different orientations of $\mathrm{C}_{60}$ observed on this surface. Bright and dim molecules show differing orientations. Reprinted with permission from [179]. Copyright (2002) by the American Physical Society.

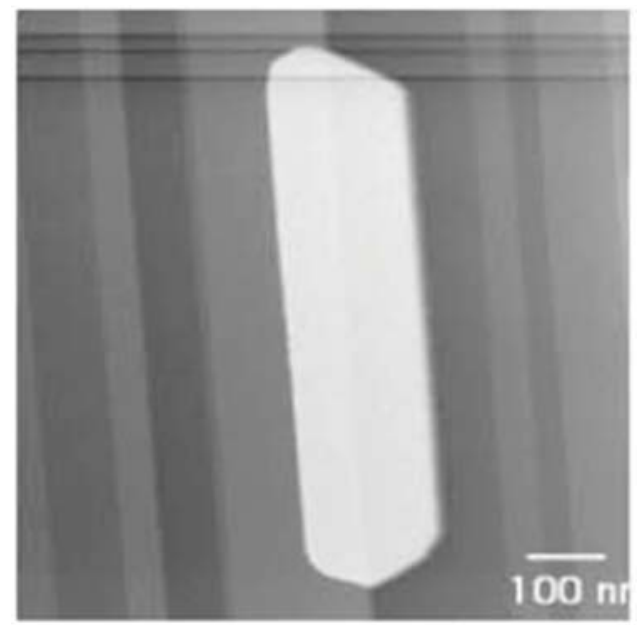

Figure 25. SFM image of the high-temperature morphology of $\mathrm{C}_{60}$ islands on $\operatorname{KBr}(001)$. Substrate temperature at deposition: $175^{\circ}$ C. Reprinted with permission from [188]. Copyright (2007) by the American Physical Society.

structures and morphologies are observed on $\mathrm{NaCl}$ [188] and on $\mathrm{CaF}_{2}$ [189].

A similar investigation of the different possible epitaxial orientations has been performed for $\mathrm{C}_{60}$ on graphite [190]. In this conductive system, low energy electron diffraction experiments showed rings indicating a lack of a definite epitaxial relationship over large scales. A theoretical analysis shows that an epitaxial relationship is energetically favorable and that several orientations of the islands are possible.

\subsection{Island morphology for larger islands}

Island morphologies observed with growth of $\mathrm{C}_{60}$ on $\mathrm{KBr}\left(\begin{array}{ll}0 & 0\end{array}\right)$ are complex. In most cases the islands consist of large parts with two molecular layers thickness, even for small coverage. It is therefore mandatory to look first at the equilibrium state 


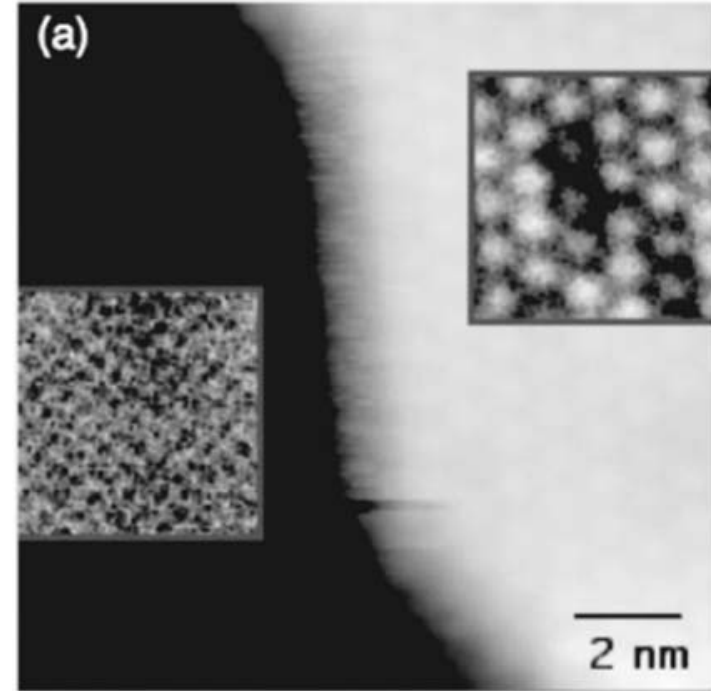

(b)

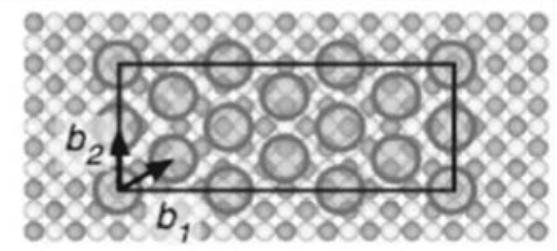

Figure 26. (a) Molecular resolution is obtained on an island of $\mathrm{C}_{60}$ molecules using SFM and in the same image, atomic resolution is obtained on the $\mathrm{KBr}(001)$ substrate. Both contrasts are highlighted with different gray-scales in the two insets. (b) From this and similar images an epitaxial relationship of the island compared to the substrate can be proposed. Reprinted with permission from [32]. Copyright (2005) by the American Physical Society.

obtained at high substrate temperatures during deposition. At high temperatures the islands have a compact shape with an edge parallel to step edges [188]. The other edges are arranged at $120^{\circ}$ angles from this edge. If all edges were equally long, the islands would have a hexagonal shape. However, the edges aligned with the step are much longer than the other four edges. For $\operatorname{KBr}(001)$ surfaces, at room temperature, branched and compact islands coexist [188]. For $\mathrm{CaF}_{2}$ substrates, it has been confirmed that the islands predominantly have a thickness of a molecular layer at low substrate temperatures [61] and two molecular layers at high substrate temperatures [189].

At low temperatures compared to the activation energy barriers (here, this refers to room temperature), branched islands dominate on $\mathrm{KBr}$, see figure 27 . The structure of these islands is similar on $\mathrm{CaF}_{2}$ and has been explained by simulations [189]. A decisive role is played by upwards hops of molecules at step edges. At high temperatures, these are frequent, and continue to fill up the second layer until it is filled. The diffusion along step edges governs the shape of truncated hexagons. At low temperatures the upward hops occur on a slower time-scale than other processes. The second layer is mainly filled by deposition of more molecules on top of the first layer. These molecules have favorable positions at the edge of the island and therefore a rim around the island is formed. For kinetic reasons, before the rim fully encloses the island, the deposition is usually finished and the island shape continues to change by dewetting processes. Such processes mainly affect the monolayer-high part of the island and leave the double-layer parts unchanged, such that the monolayer parts are consumed and complex structures are formed. A more complete overview on cluster growth and the molecular processes that lead to particular structures is given in [191].

The growth of $\mathrm{C}_{60}$ on graphite is strongly opposed to the growth on $\operatorname{KBr}(001)$, see $[192,193]$. For graphite, the first layer forms compact islands while starting from the second layer, branched structures are formed. The branched structures arise through a diffusion-limited growth mode and are of fractal nature [192]. Molecules located on top of a $\mathrm{C}_{60}$ island are strongly limited in mobility compared to the molecules on the graphite substrate. Similar growth is also observed on $\mathrm{SiC}$ [194].

The formation of branched islands can also be regarded as being a result of different surface energies. A comparison between $\mathrm{C}_{60}$ islands on different materials of different surface energies show that, as expected, at larger surface energies, a wetting layer is formed (Stranski-Krastanov growth) while at smaller surface energies, islands are preferred (Volmer-Weber growth) $[21,85]$. While on metals, compact flat islands with a large contact area between molecular islands and surface dominate due to their large surface energies, on insulators three-dimensional branched islands dominate. The dependence of the molecule-substrate interface energy on the different substrates is not considered in this comparison. It is implicitly assumed that the molecule-substrate energy has a given relationship to the substrate surface energy.

The picture becomes more detailed, but still strongly simplified, if one considers that together with energetic considerations diffusion governs the formation of molecular structures. In general, we expect that a large adsorption energy should be correlated with deep potential wells for the individual molecules and thus, slow diffusion. Here, we have two cases where the diffusion properties of molecules on the substrate compared to the diffusion properties of molecules on the molecular islands are inversed with respect to the adsorption energies: for $\mathrm{C}_{60}$ on $\operatorname{KBr}(001)$ the energy of a molecule on top of a molecular layer is more favorable compared to the substrate and thus the molecules preferentially grow a second layer. Diffusion on the substrate is comparable to diffusion on the island. In contrast for $\mathrm{C}_{60}$ on graphite, diffusion on the substrate is faster compared to diffusion on the island. These considerations are summarized in figure 28.

$\mathrm{C}_{60}$ island formation and dewetting has been compared on different ionic crystalline as well as a metal surface by using the surface energy as a parameter [85], see figure 29. The idea is to quantify the interaction of the molecule with these surfaces by just one parameter, the surface energy. Low surface energy materials are expected to show dewetting and high surface energy materials are expected to show wetting. Here, we attempt to add graphite to the picture. Its surface energy is even lower than the one cited for the lowest energy mentioned in figure 29, with a value of only $54.8 \mathrm{~mJ} \mathrm{~m}^{-2}$ [195]. However, even though $\mathrm{C}_{60}$ shows dewetting on $\operatorname{KBr}(001)$ [32], on graphite it shows wetting comparable to the observations on $\mathrm{CaCO}_{3}(1014)$ which is reported to have a surface energy of $590 \mathrm{~mJ} \mathrm{~m}^{-2}$. This shows that the surface energy alone cannot fully describe the interaction of molecules with a surface and that the detailed nature of the interaction should 


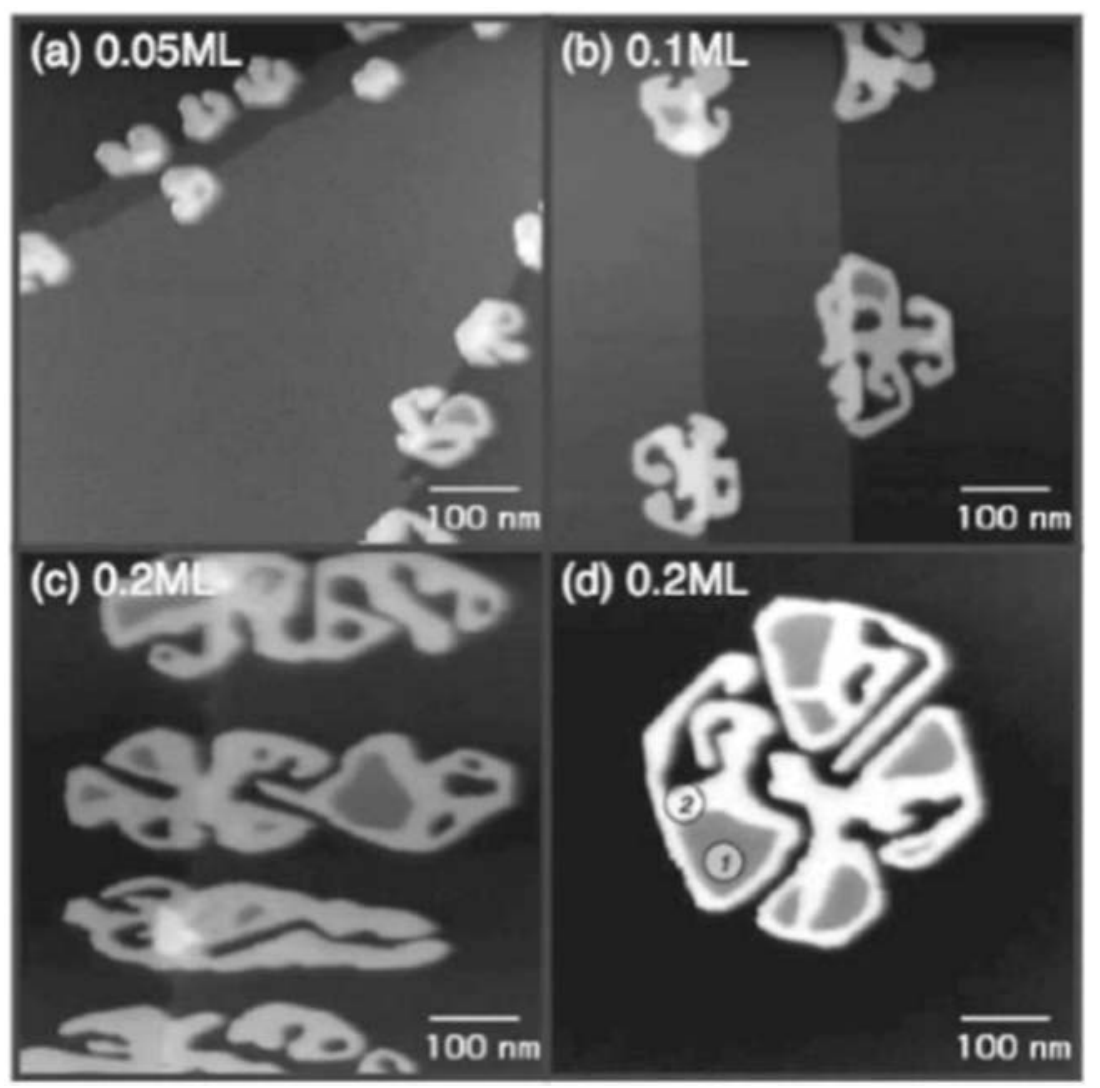

Figure 27. SFM image of $\mathrm{C}_{60}$ molecular islands on $\operatorname{KBr}(001)$ taken at different positions on the sample. In (d) regions with one molecular layer height are marked with 1 , and regions with two molecular layers height are marked with 2. Reprinted with permission from [32]. Copyright (2005) by the American Physical Society.

a)

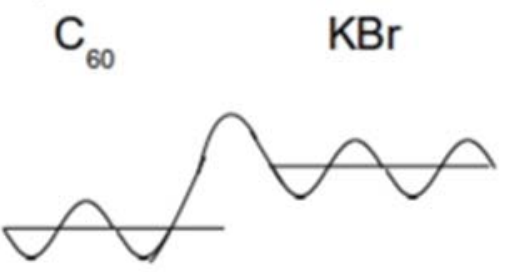

b)
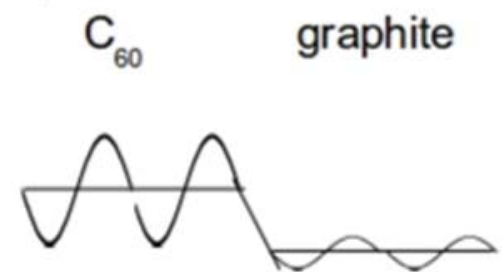

Figure 28. (a) The situation that diffusion remains approximately at the same speed, while the adsorption energy is much larger on the molecular island is summarized as for the situation in $\mathrm{C}_{60}$ growth on $\mathrm{KBr}$. (b) The diffusion of $\mathrm{C}_{60}$ is much faster for the molecules on the graphite surface compared to the molecular island although the adsorption energy on graphite is larger.
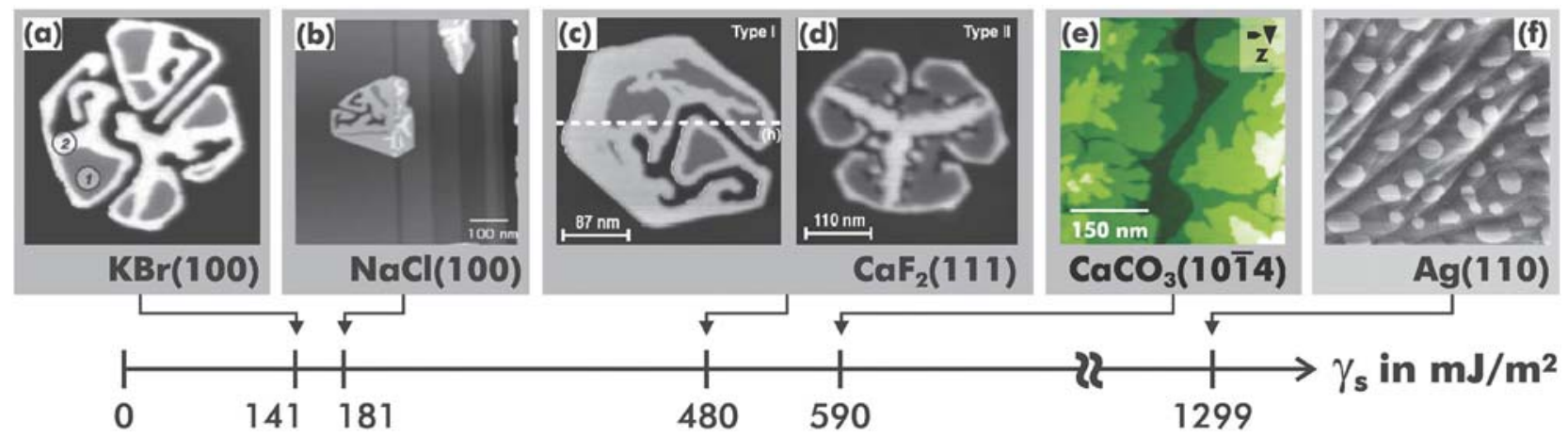

Figure 29. $\mathrm{C}_{60}$ adsorbed structures on different surfaces put into a relation by citing their surface energies. (a)—(d) Dewetted islands observed on ionic crystalline surfaces imaged by SFM. (e) Wetting is observed on calcite imaged by SFM. (f) Single molecular layer islands on the metallic $\operatorname{Ag}\left(\begin{array}{ll}1 & 1\end{array}\right)$ surface imaged by STM. Reproduced from [85] with permission of The Royal Society of Chemisty. 
a)

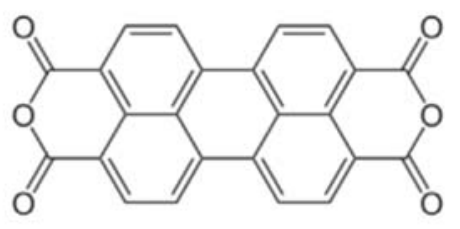

b)

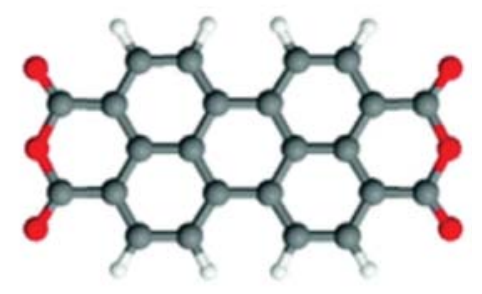

Figure 30. Structure of a PTCDA molecule. (a) Chemical notation. (b) Atomic representation: oxygen atoms are depicted in red.

be considered rather than the surface energy of the substrate alone. The surface energy of the substrate is only a well-chosen parameter if the class of materials is not changed, e.g. for comparisons of different ionic crystalline surfaces.

\section{PTCDA}

\subsection{Overview}

3,4,9,10-perylenetetracarboxylic dianhydride, here referred to as PTCDA for simplicity, is a derivative of perylene which is also a nanographene, but wider compared to pentacene, see figure 30. In PTCDA additional oxygen atoms are located at the ends of perylene. The presence of oxygen strongly modifies the charge distribution in the molecule compared to perylene. Due to the twofold symmetric arrangement of the charge, the molecule possesses no dipole moment, but a quadrupolar moment.

Compared to the previously described molecules pentacene and $\mathrm{C}_{60}$, where weaker charge effects are expected, the force field above PTCDA is strongly governed by the electrostatic fields generated by the quadrupolar moment. The role of electrostatic forces on imaging molecules with strong spatially dependent charge distributions, in this case acetate and triflouroacetate, has been investigated previously [196]. PTCDA has first been atomically resolved using STM on $\mathrm{Cu}(110)$ [197]. For an overview of PTCDA structures on noble metal surfaces, see [198, 199]. Confined PTCDA molecules in $\mathrm{KBr}$ pits have been investigated first on a surface with atomic pits intentionally created by electron irradiation [30]. It was found that the molecules primarily attach to step edges, see figure 31 .

PTCDA is evaporated at a temperature of $300^{\circ} \mathrm{C}$. For more details on the preparation process for many of the data shown here, see [33].

\subsection{Growth mode}

Large images (figure 32 ) show steps on the $\operatorname{KBr}(001)$ surface and molecular islands with the shape of truncated pyramids.
The $\mathrm{KBr}(100)$ and $(010)$ directions are aligned with the edges of the image. The $\operatorname{KBr}(001)$ surface shows structures characteristic for cleaving, in particular a large wedge-shaped structure running from top left to bottom right with the tip located at the bottom right. It also shows mostly square structures oriented in the (100) and (010) directions of $\mathrm{KBr}$ presumably resulting from the onset of desorption of $\mathrm{KBr}$ during heating. Most of the PTCDA crystallites have six different facets with angles of nearly $120^{\circ}$ between the orientations of these facets. Some of the PTCDA islands are aligned with substrate $(010)$ directions, but there is variability in the orientation of the island edges pointing to a rather small molecule-substrate interaction, as expected, with several possible epitaxial relationships. Instead, the islands are often located at lower terraces and aligned with step edges indicating that the growth is more strongly influenced by the templating effect of steps than by epitaxy.

The height of the islands varies from one up to about 30 molecular layers. It is known and there are also indications from figure 33 that PTCDA crystals arranges in molecular layers parallel to the surface. Figure 33 shows part of such a crystallite imaged with high resolution. On two of the facets molecular resolution is clearly observed. A more detailed analysis (see figure 34 below) shows that also the top of the island is molecularly resolved. From such images the number of molecular layers in an island can be counted. The height of this island is $27-28$ molecular layers. Some ambiguities arise at the interface of the island with the $\mathrm{KBr}$ substrate possibly either due to the structure of the first molecular layer on the substrate or due to the convolution with the tip shape with a radius of below $10 \mathrm{~nm}$. The third facet is steep and governed by the tip shape rather than the sample shape as a result of convolution effects. The measurement shows that the tip used for this experiment is indeed sharp with an opening angle well below $55^{\circ}$, as specified by the manufacturer.

The overall appearance of the islands is elongated. This shape of the island arises from the herringbone phase arrangement of the molecules. From the topography of the surface itself it is not clear whether the islands occur on a wetting layer or directly on the substrate. Since the molecule-surface interaction is rather weak, forming a wetting layer is unfavorable for energetic reasons, so a wetting layer is not expected. For $\mathrm{NaCl}(001)$ substrates, a dewetting transition has been observed. Before dewetting, the substrate is covered by molecules. Through dewetting, bulk phase islands grow. In the vicinity of the islands the substrate area has been imaged in the same image as the molecular islands and showed the $\mathrm{NaCl}$ structure [86], confirming the local absence of a wetting layer. On $\mathrm{NaCl}$, three different molecular arrangements have been found, one of which is bulk-like [200].

Similar islands had been observed before at much larger length scales ( $>3 \mu \mathrm{m}$ image size) using tapping mode SFM not only on $\mathrm{KBr}$ but also on $\mathrm{NaCl}$ and $\mathrm{KCl}$ [201]. From obtaining molecular resolution on several facets of a PTCDA island on metal surfaces $(\mathrm{Cu}(110), \mathrm{Cu}(111)$ and $\mathrm{Au}(111))$, the three dimensional structure of an island has been determined and the structures of several islands were compared [202]. All crystals showed a layered structure with layers parallel to 


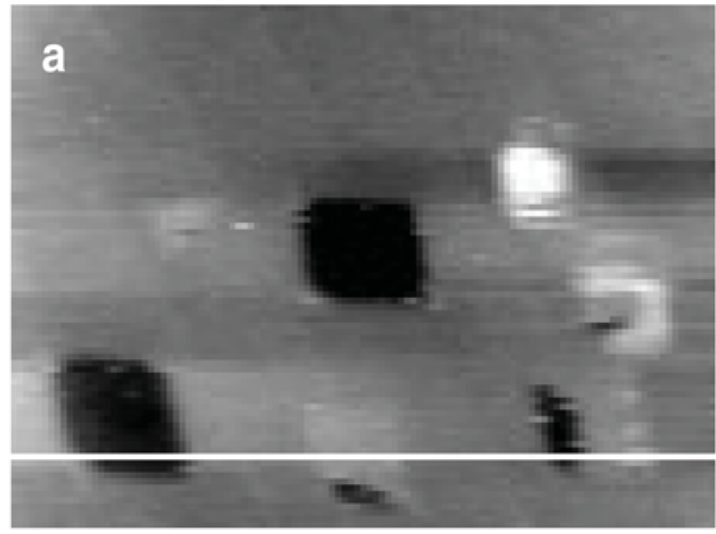

C

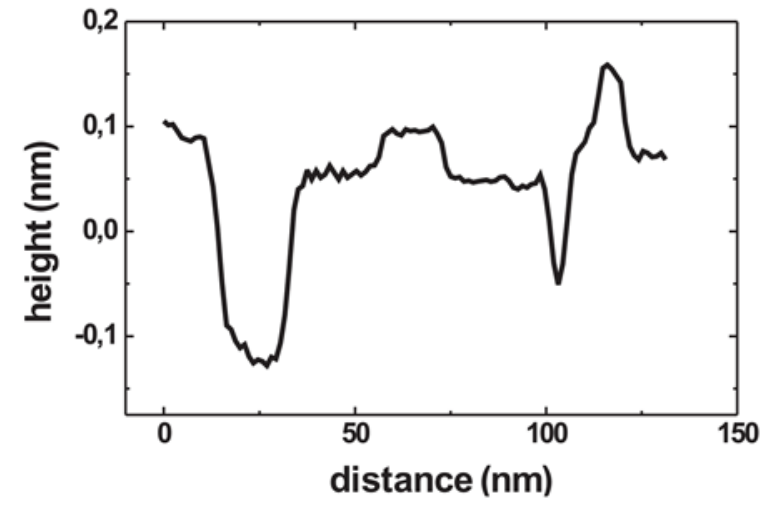

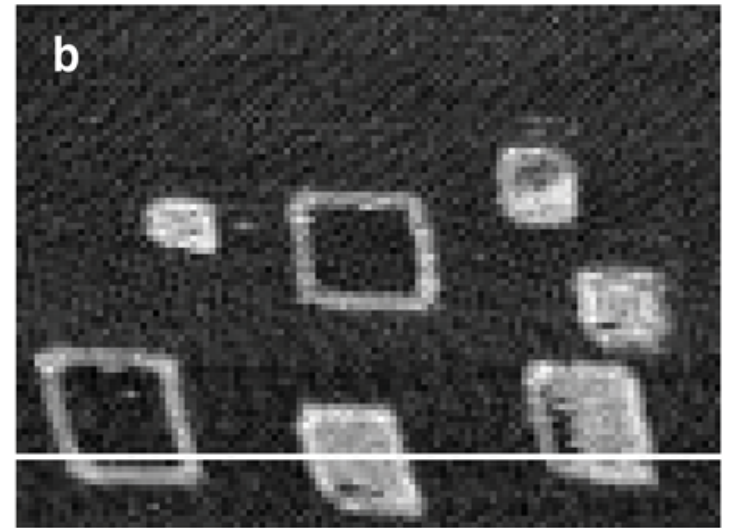

d

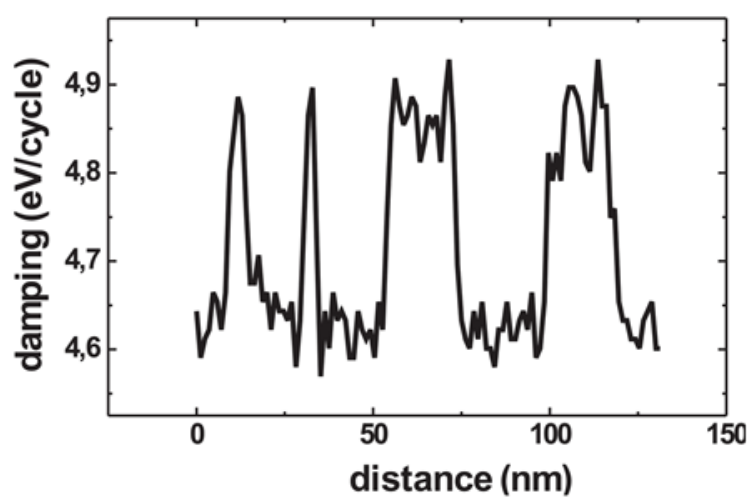

Figure 31. (a) Topographic dynamic mode SFM image of PTCDA on a electron-irradiated KBr(00 1) surface. (b) Simultaneously recorded energy dissipation image. (c) and (d) show line cuts at the position of the white lines in (a) and (b). Reproduced from [30]. (C) IOP Publishing Ltd. All rights reserved.

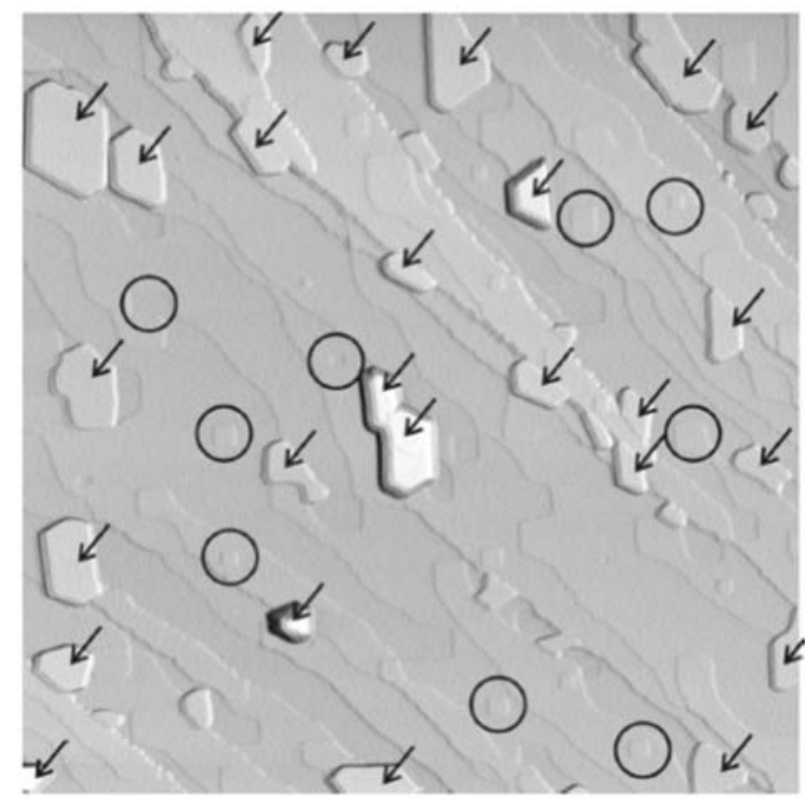

Figure 32. SFM overview image of a partly PTCDA-covered $\mathrm{KBr}(001)$ surface. Most PTCDA islands are marked with arrows. The circles accentuate square or rectangular structures resulting from $\mathrm{KBr}$. Image size: $2 \mu \mathrm{m} \times 2 \mu \mathrm{m}$. Coverage: 0.3 monolayers. Reprinted with permission from [33]. Copyright (2005) by the American Physical Society. the substrate surface. However, the relative displacement of subsequent layers with respect to each other differed for the crystals investigated.

On glass substrates, the growth process has been analyzed using scaling theory [203]. In this theory, the surface roughness is analyzed mathematically with the help of the heightheight correlation function. The dependence of this function on time and the distance below or above the correlation length is described by power laws and offers insight about the growth mechanism. Scaling laws indicate an upward mound growth mechanism, with the measured scaling exponents indicating an anomalous behavior similar to growth of pentacene [131]. This growth mode is characterized by the competition of diffusion and high step-edge barriers.

Growth of PTCDA on NaCl-covered $\mathrm{Cu}(111)$ shows that PTCDA preferentially grows on $\mathrm{Cu}$, but the structure is altered compared to pure $\mathrm{Cu}$ surfaces [204]. It is suggested that the observed rod structures arise from the formation of a $\mathrm{Na}_{x}$-PTCDA complex. Some molecules attach to vacancies at $\mathrm{NaCl}$ steps. For PTCDA on $\mathrm{Ag}(111)$, also structures differing in their vertical stacking (called $\alpha$ and $\beta$-like structures) are found [205]. PTCDA grows here in the Stranski-Krastanov growth mode with a morphology transition as a function of substrate temperature during growth. This morphology transition concerns the way the rectangular bcc lattice of PTCDA is aligned with the substrate $\left(\operatorname{Ag}\left(\begin{array}{lll}1 & 1 & 1\end{array}\right)\right)$ hexagonal fcc lattice. At 


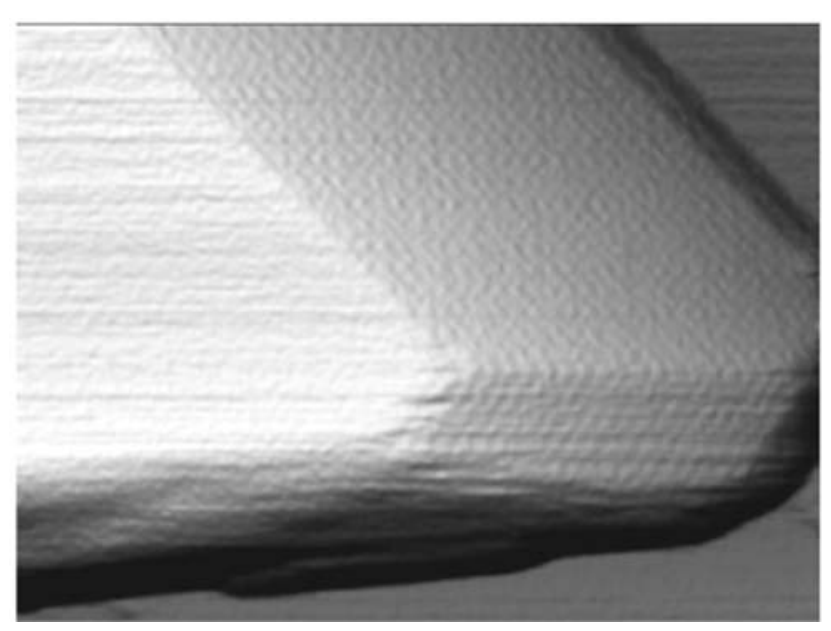

Figure 33. Part of the top surface and three different facets of a PTCDA crystallite on a $\operatorname{KBr}(001)$ surface is imaged with high resolution SFM. Two of the facets are clearly resolved at the molecular level, a closer analysis shows that molecular resolution is also obtained at the top surface. Image size: $80 \mathrm{~nm} \times 60 \mathrm{~nm}$. Reprinted with permission from [33]. Copyright (2005) by the American Physical Society.

low substrate temperatures, the fcc $(1 \overline{1} 0)$ is aligned parallel to the bcc(001) direction of PTCDA (Nishiyama-Wassermann orientation), whereas at high substrate temperatures, the rectangular unit cell is aligned with one of the diagonals, i.e. fcc (1 $1 \overline{1} 0)$ is parallel to bcc $(1 \overline{1} \overline{1})$ (Kurdjumov-Sachs orientation). Films deposited at high substrate temperatures are stable while films deposited at low substrate temperatures are metastable [206]. A line-like superstructure was found for PTCDA on $\operatorname{Ag}(111)$ using SFM [207].

\subsection{Molecular resolution}

Molecular resolution has been obtained on the facet of a molecular island (figure 34). The experimentally obtained image was compared to a simulation using potentials from a standard database. The van-der-Waals interaction was treated using Lennard-Jones potentials. Even more details are resolved compared to molecular resolution images obtained on a $\mathrm{KBr}$ ultrathin film on $\mathrm{Ag}(111)$ [208]. For typical measurements with intramolecular resolution, the two edges in the long direction of PTCDA look somewhat brighter than the body of the molecule as has been observed for PTCDA islands confined to nanoscale $\mathrm{KBr}$ pits [209]. These experimental results were further investigated using first-principle simulations [68]. The experimental observation that molecules are trapped at kink sites was only reproduced by the simulations if a first-principles treatment of the van-der-Waals force was included. It was found that the van-der-Waals force dominates molecular binding for PTCDA on KBr. Also for PTCDA on rutile $\mathrm{TiO}_{2}\left(\begin{array}{lll}1 & 1 & 0\end{array}\right)$ it was found that the van-der-Waals (dispersion) force play a dominant role in chemisorption [210]. On a $\mathrm{Cu}(111)$ surface it was found that intramolecular contrast was only observed in the second layer of molecules [211]. This intramolecular contrast is in agreement with the molecule's internal charge distribution.

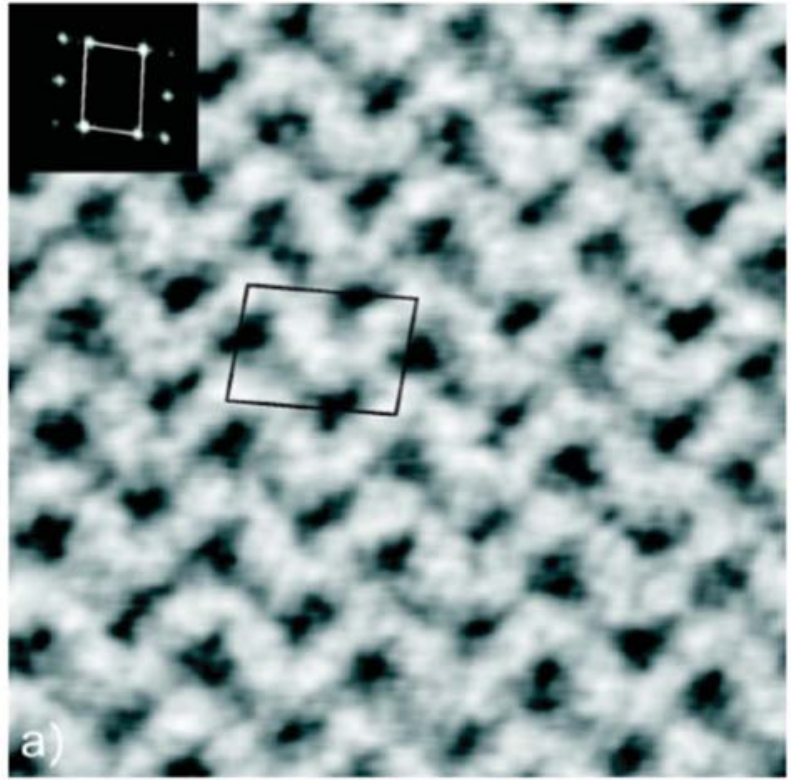

Figure 34. Molecular resolution SFM image of PTCDA obtained on the top of a crystallite on the $\operatorname{KBr}(001)$ surface. Image size approx $10 \mathrm{~nm} \times 10 \mathrm{~nm}$. Reprinted with permission from [33]. Copyright (2005) by the American Physical Society.

In the repulsive regime, where atomic resolution is obtained for a molecule, a simplified model has been proposed that allows to calculate the contribution of Pauli repulsion [213]. In this regime the forces are a measure of the electron density distribution in the molecule. In agreement with this assertion, at close tip-sample distances in horizontal cuts of three-dimensional force-field measurement, an image corresponding to the electron density distribution is obtained [214]. Force-field measurements on the $\mathrm{Au}(111)$ surface, where the tip was attaching to a PTCDA molecule that was lifted together with the tip and where the tip oscillation amplitude was only $0.02 \mathrm{~nm}$-much smaller than the length of the molecule (1.4 nm) — showed several features upon approach [215]: first, attraction was observed, followed by a sharp repulsive peak. These features arise from the contact of the small edge of the molecule with the surface, where the molecule has an extended configuration between tip and surface. Then the interaction weakens until, 0.7 to $1.1 \mathrm{~nm}$ closer to the surface more features are observed, presumably due to a configuration where the molecule is in a flat-lying configuration between tip and surface [216]. For PTCDA on $\mathrm{Ag} / \mathrm{Si}(1111)-(\sqrt{3} \times \sqrt{3})$, intramolecular contrast was obtained, see figure 35 .

Also energy dissipation processes in SFM were described in detail for PTCDA on Ag [217]. Bistabilities occur in the potential-energy landscape during formation and breaking of a bond between PTCDA molecules on the surface and PTCDA molecules on the tip. The force during approach differs from the one measured during retraction. The hysteresis explains the measured energy loss. For similar measurements, with the help of calculations, an Ag-contaminated Si tip is identified as the most likely tip termination [218]. The energy dissipation can be explained as adhesion hysteresis involving a motion of the rather mobile oxygen groups at the side of the molecule and the more rigid perylene core. 


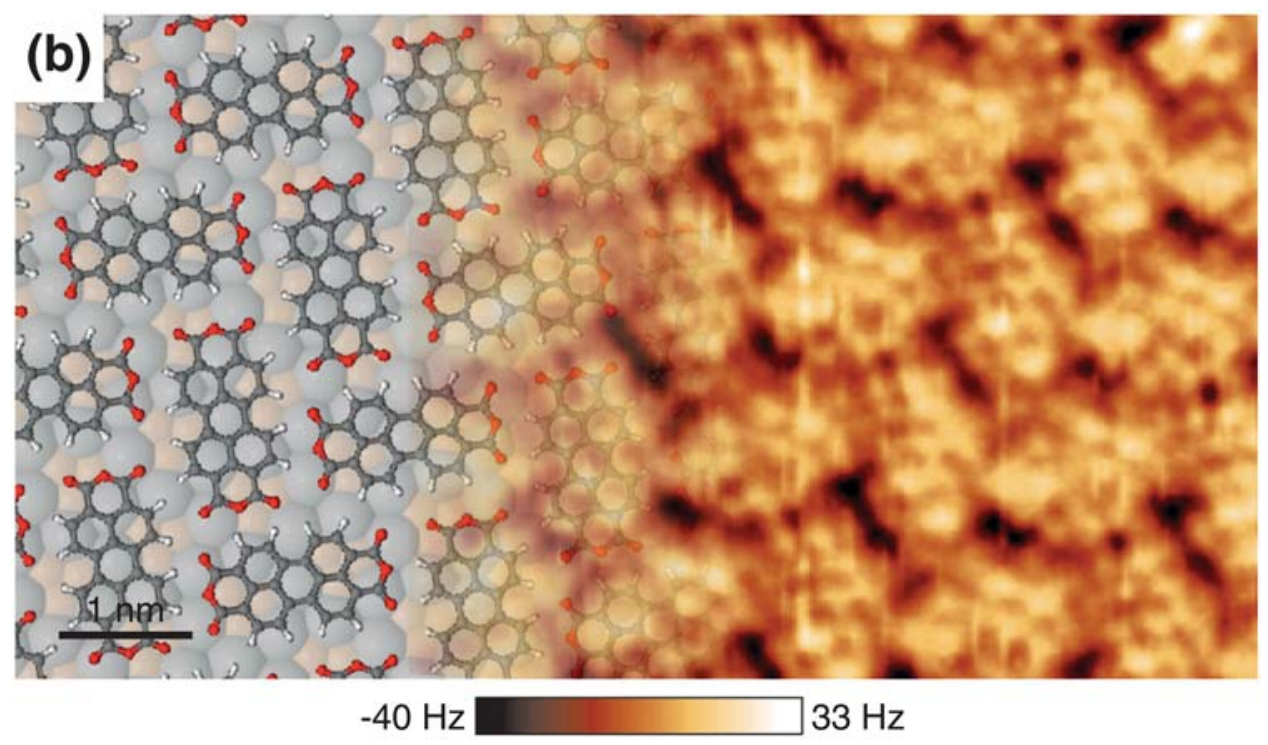

Figure 35. Constant height dynamic mode SFM image of PTCDA on a $\mathrm{Ag} / \mathrm{Si}(111)-(\sqrt{3} \times \sqrt{3})$ surface. Reprinted with permission from [212]. Copyright (2015) by the American Physical Society.

Similar molecular resolution images were obtained for PTCDA on KCl together with optical spectra [219]. The optical spectra were found to be particularly narrow for a metastable square phase on this surface. PTCDA has also been studied on $\mathrm{NaCl}$, both by SFM [86] and by spot profile analysis low energy electron diffraction (SPA-LEED) [220] where the same structure was found. The structure alters by dewetting as a function of time. On $\mathrm{Au}(111)$, PTCDA forms a variety of phases stabilized by hydrogen bonding, amongst them are different herringbone and square phases studied by STM and DFT calculations [70]. In contrast, on $\operatorname{Ag}(110)$ the molecules lie flat on the surface, oriented in the (001) direction of the surface unit cell, in a commensurate structure [221]. On $\operatorname{InSb}(001)$ PTCDA forms stripe-like structures with the long-axes of the molecules oriented in the [1 110$]$ direction of the substrate [222].

\section{Conclusions}

A scalable alternative to current device fabrication in molecular electronics relies on single molecules or molecular islands on insulating surfaces, contacted by planar metallic or other contacts. Designing working devices in this arrangement requires a detailed understanding of the interaction of molecules with insulating surfaces. While a number of STM studies of molecules on metallic surfaces have contributed to understanding molecules on metals, molecules on bulk insulators have received relatively little attention so far. Molecules on insulating surface are also interesting for their fundamentally different and much weaker interaction with the substrates. Due to this weak interaction, the moleculemolecule interaction becomes more important compared to metal surfaces. Here we have focused on alkali halides or earth alkali halides as substrates. Nanographenes, such as pentacene, $\mathrm{C}_{60}$ or the oxygen-modified PTCDA have electronic properties that make them candidates for molecular electronics, in particular pentacene is known for its extraordinary carrier mobility. Studying and finally tailoring the structure of the molecular islands is important to understand and influence the anisotropic electronic transport and dewetting properties of pentacene. Studies of the local work function with the Kelvin method show different values of the work function for different molecular orientations. Such studies also reveal that line defects show a locally different work function that could lead to charge trapping. $\mathrm{C}_{60}$ is nearly isotropic in contrast to pentacene and PTCDA, but has peculiar dewetting properties that cause the molecules to move to the second molecular island. $\mathrm{C}_{60}$ nucleates at step edges with a single molecular nucleus that can be imaged with SFM despite its local mobility. Molecular islands show additional stability to the force applied by the tip, when they are composed of a magic numbers of molecules. For the bulk insulator PTCDA, the elongated islands can be molecularly resolved even on the facets such that the molecular arrangement in the islands is studied with great detail. The islands are composed of layers of molecules arranged in a herringbone phase.

\section{Acknowledgments}

Ithank Sarah Burke, Michael Marz, Jeffrey Mativetsky, Carmen Pérez León, Sebastian Schneider and Hilbert von Löhneysen for critically reading the manuscript and for discussions and support. Financial support from the European Research Council through the Starting Grant NANOCONTACTS (No. 239838) and the Brigitte-Schlieben-Lange program of the Ministry of Science, Research and Arts, Baden-Württemberg, is gratefully acknowledged. 


\section{ORCID iDs}

R Hoffmann-Vogel (1) https://orcid.org/0000-0003-4984-6210

\section{References}

[1] Gershenson M E, Podzorov V and Morpurgo A F 2006 Colloquium: Electronic transport in single-crystal organic transistors Rev. Mod. Phys. 78973

[2] Aviram A and Ratner M A 1974 Molecular rectifiers Chem. Phys. Lett. 29277

[3] Barth J V 2007 Molecular architectonic on metal surfaces Annu. Rev. Phys. Chem. $\mathbf{5 8} 375$

[4] Rosei F, Schunack M, Naitoh Y, Jiang P, Gourdon A, Laegsgaard E, Stensgaard I, Joachim C and Besenbacher F 2003 Properties of large organic molecules on metal surfaces Prog. Surf. Sci. 7195

[5] Komeda T 2005 Chemical identification and manipulation of molecules by vibrational excitation via inelastic tunneling process with scanning tunneling microscopy Prog. Surf. Sci. 7841

[6] Grill L 2008 Functionalized molecules studied by STM: motion, switching and reactivity J. Phys.: Condens. Matter 20053001

[7] Gao H-J and Gao L 2010 Scanning tunneling microscopy of functional nanostructures on solid surfaces: manipulation, self-assembly and applications Prog. Surf. Sci. 8528

[8] Klappenberger F 2014 Two-dimensional functional molecular nanoarchitectures-complementary investigations with scanning tunneling microscopy and $\mathrm{x}$-ray spectroscopy Prog. Surf. Sci. 891

[9] Venkataraman L, Klare J E, Nuckolls C, Hybertsen M S and Steigerwald M L 2006 Dependence of single-molecule junction conductance on molecular conformation Nature 442904

[10] Neel N, Kröger J, Limot L, Frederiksen T, Brandbyge M and Berndt R 2007 Controlled contact to a $\mathrm{C}_{60}$ molecule Phys. Rev. Lett. 98065502

[11] Lafferentz L, Ample F, Yu H, Hecht S, Joachim C and Grill L 2009 Conductance of a single conjugated polymer as a continuous function of its length Science $\mathbf{3 2 3} 1193$

[12] Schmaus S, Bagrets A, Nahas Y, Yamada T K, Bork A, Bowen M, Beaurepaire E, Evers F and Wulfhekel W 2011 Giant magnetoresistance through a single molecule Nat. Nanotechnol. 6185

[13] Fournier N, Wagner C, Weiss C, Temirov R and Tautz F S 2011 Force-controlled lifting of molecular wires Phys. Rev. B 84035435

[14] Haick H and Cahen D 2008 Making contact: connecting molecules electrically to the macroscopic world Prog. Surf. Sci. 83217

[15] Hirose M, Tsunemi E, Kobayashi K and Yamada H 2013 Influence of grain boundary on electrical properties of organic crystalline grains investigated by dual-probe atomic force microscopy Appl. Phys. Lett. 103173109

[16] Kelley T W and Frisbie C D 2001 Gate voltage dependent resistance of a single organic semiconductor grain boundary J. Phys. Chem. B 1054538

[17] Yang H, Shin T J, Ling M-M, Cho K, Ryu C Y and Bao Z 2005 Conducting AFM and 2D GIXD studies on pentacene thin films J. Am. Chem. Soc. 12711542

[18] Al-Mahboob A, Sadowski J T, Fujikawa Y, Nakajima K and Sakurai T 2008 Kinetics-driven anisotropic growth of pentacene thin films Phys. Rev. B 77035426
[19] Stadler C, Hansen S, Kröger I, Kumpf C and Umbach E 2009 Tuning intermolecular interaction in long-range-ordered submonolayer organic films Nat. Phys. 5153

[20] Bonn D, Eggers J, Indekeu J, Meunier J and Rolley E 2009 Wetting and spreading Rev. Mod. Phys. 81739

[21] Burke S A, Topple J M and Grütter P 2009 Molecular dewetting on insulators J. Phys.: Condens. Matter 21423101

[22] Guggisberg M, Bammerlin M, Loppacher C, Pfeiffer O, Abdurixit A, Barwich V, Bennewitz R, Baratoff A, Meyer E and Güntherodt H-J 2000 Separation of interactions by noncontact force microscopy Phys. Rev. B 6111151

[23] Bammerlin M, Lüthi R, Meyer E, Baratoff A, Lü J, Guggisberg M, Loppacher C, Gerber C and Güntherodt H-J 1998 Dynamic SFM with true atomic resolution on alkali halide surfaces Appl. Phys. A 66 S293

[24] Morita S, Wiesendanger R and Meyer E (ed) 2002 Noncontact Atomic Force Microscopy (Berlin: Springer)

[25] Barth C, Foster A S, Henry C R and Shluger A L 2011 Recent trends in surface characterization and chemistry with high-resolution scanning force methods Adv. Mater. 23477

[26] Loppacher C, Guggisberg M, Pfeiffer O, Meyer E, Bammerlin M, Lüthi R, Schlittler R, Gimzewski J K, Tang H and Joachim C 2003 Direct determination of the energy required to operate a single molecule switch Phys. Rev. Lett. 90066107

[27] Gotsmann B, Schmidt C, Seidel C and Fuchs H 1998 Molecular resolution of an organic monolayer by dynamic AFM Eur. Phys. J. B 4267

[28] Butt H-J, Seifert K and Bamberg E 1993 Imaging molecular defects in alkanethiol monolayers with an atomic force microscope J. Phys. Chem. 977316

[29] Fukuma T, Kobayashi K, Noda K, Ishida K, Horiuchi T, Yamada H and Matsushige K 2002 Molecular-scale noncontact AFM studies of ferroelectric organic thin films epitaxially grown on alkali halides Surf. Sci. 516103

[30] Nony L, Bennewitz R, Pfeiffer O, Gnecco E, Baratoff A, Meyer E, Eguchi T, Gourdon A and Joachim C 2004 $\mathrm{Cu}-\mathrm{TBPP}$ and PTCDA molecules on insulating surfaces studied by ultra-high-vacuum non-contact AFM Nanotechnology 15 S91

[31] Tanaka S, Suzuki H, Kamikado T and Mashiko S 2004 Conformational study of porphyrin-based molecules using non-contact atomic force microscopy Nanotechnology 15 S 87

[32] Burke S A, Mativetsky J M, Hoffmann R and Grütter P 2005 Nucleation and submonolayer growth of $\mathrm{C}_{60}$ on $\mathrm{KBr}$ Phys. Rev. Lett. 94096102

[33] Kunstmann T, Schlarb A, Fendrich M, Wagner T, Möller R and Hoffmann R 2005 Dynamic force microscopy study of 3,4,9,10-perylenetetracarboxylic dianhydride on $\operatorname{KBr}\left(\begin{array}{lll}0 & 0 & 1\end{array}\right)$ Phys. Rev. B 71121403

[34] Hoffmann R and Hermann B A 2012 Scanning probe microscopy Analytical Methods in Supramolecular Chemistry ed C A Schalley (Berlin: Wiley)

[35] Rahe P, Kittelmann M, Neff J L, Nimmrich M, Reichling M, Maass P and Kühnle A 2013 Tuning molecular selfassembly on bulk insulator surfaces by anchoring of the organic building blocks Adv. Mat. 253948

[36] Gross L, Mohn F, Moll N, Liljeroth P and Meyer G 2009 The chemical structure of a molecule resolved by atomic force microscopy Science 3251110

[37] Weiss C, Wagner C, Kleimann C, Rohlfing M, Tautz F S and Temirov R 2010 Imaging pauli repulsion in scanning tunneling microscopy Phys. Rev. Lett. 105086103 
[38] Zhu X-Y 2004 Electronic structure and electron dynamics at molecule-metal interfaces: implications for molecule-based electronics Surf. Sci. Rep. 561

[39] Koch N 2008 Energy levels at interfaces between metals and conjugated organic molecules J. Phys.: Condens. Matter 20184008

[40] Moth-Poulsen K and Bjørnholm T 2009 Molecular electronics with single molecules in solid-state devices Nat. Nanotechnol. 4552

[41] Nimmrich M, Kittelmann M, Rahe P, Harneit W, Mayne A J, Dujardin G and Kühnle A 2012 Influence of charge transfer doping on the morphologies of $\mathrm{C}_{60}$ islands on hydrogenated diamond C(100)- $(2 \times 1)$ Phys. Rev. B 85035420

[42] Nikiforov M P, Zerweck U, Milde P, Loppacher C, Park TH, Uyeda H T, Eng L and Bonnell D 2008 The effect of molecular orientation on the potential of porphyrin-metal contacts Nano Lett. 8110

[43] Neff J L, Milde P, Pérez León C, Kundrat M D, Eng L M, Jacob C R and Hoffmann-Vogel R 2014 Epitaxial growth of pentacene on alkali halide surfaces studied by Kelvin probe force microscopy ACS Nano 83294

[44] Yogev S, Halpern E, Matsubara R, Nakamura M and Rosenwaks Y 2011 Direct measurement of density of states in pentacene thin film transistors Phys. Rev. B 84165124

[45] Jaquith M, Muller E M and Marohn J A 2007 Timeresolved electric force microscopy of charge trapping in polycrystalline pentacene J. Phys. Chem. B 1117711

[46] Tello M, Chiesa M, Duffy C M and Sirringhaus H 2008 Charge trapping in intergrain regions of pentacene thin film transistors Adv. Funct. Mater. 183907

[47] Jia Q, Hu Z-X, Ji W, Burke S A, Gao H-J, Grütter P and Guo H 2016 Adsorption of PTCDA and $\mathrm{C}_{60}$ on $\operatorname{KBr}(001)$ : electrostatic interaction versus electronic hybridization Phys. Chem. Chem. Phys. 1811008

[48] Heim T, Lmimouni K and Vuillaume D 2004 Ambipolar charge injection and transport in a single pentacene monolayer island Nano Lett. 42145

[49] Hoff R, Henry C R and Barth C 2016 Charging $\mathrm{C}_{60}$ islands with the AFM tip Nanoscale 8411

[50] Whitesides G M and Grzybowski B 2002 Self-assembly at all scales Science 2952418

[51] Lehn J-M 2002 Toward self-organization and complex matter Science 2952400

[52] Kling F, Bechstein R, Rahe P and Kühnle A 2015 Selfassembly of organic molecules on insulating surfaces Noncontact Atomic Force Microscopy vol 3, ed S Morita et al (Berlin: Springer)

[53] Berner S, Brunner M, Ramoino L, Suzuki H, Güntherodt H-J and Jung T A 2001 Time evolution analysis of a 2D solidgas equilibrium: a model system for molecular adsorption and diffusion Chem. Phys. Lett. 348175

[54] Zangwill A 1988 Physics at Surfaces (Cambridge: Cambridge University Press)

[55] Gehrig J C, Penedo M, Parschau M, Schwenk J, Marioni M A, Hudson E W and Hug H J 2017 Surface single-molecule dynamics controlled by entropy at low temperatures Nat. Commun. 814404

[56] Henzler M and Göpel W 1994 Oberflächenphysik des Festkörpers (Stuttgart: Teubner)

[57] Cai J et al 2010 Atomically precise bottom-up fabrication of graphene nanoribbons Nature 466470

[58] Kittelmann M, Rahe P, Nimmrich M, Hauke C M, Gourdon A and Kühnle A 2011 On-surface covalent linking of organic building blocks on a bulk insulator ACS Nano $\mathbf{5} 8420$

[59] Hooks D E, Fritz T and Ward M D 2001 Epitaxy and molecular organization on solid substrates Adv. Mater. 13227

[60] Brune H, Bales G S, Jacobsen J, Boragno C and Kern K 1999 Measuring surface diffusion from nucleation island densities Phys. Rev. B 605991
[61] Loske F, Lübbe J, Schütte J, Reichling M and Kühnle A 2010 Quantitative description of $\mathrm{C}_{60}$ diffusion on an insulating surface Phys. Rev. B $\mathbf{8 2} 155428$

[62] Mativetsky J M, Miyahara Y, Fostner S, Burke S A and Grütter P 2007 Use of an electron-beam evaporator for the creation of nanostructured pits in an insulating surface Appl. Phys. Lett. 88233121

[63] Nony L, Gnecco E, Baratoff A, Alkauskas A, Bennewitz R, Pfeiffer O, Maier S, Wetzel A, Meyer E and Gerber C 2004 Observation of individual molecules trapped on a nanostructured insulator Nano Lett. 42185

[64] Schwoebel R L 1969 Step motion on crystal surfaces. II J. Appl. Phys. 40614

[65] Desiraju G R and Steiner T 1999 The Weak Hydrogen Bond in Structural Chemistry and Biology (Oxford: Oxford University Press)

[66] Desiraju G R, Vittal J J and Ramanan A 2011 Crystal Engineering: a Textbook (Hackensack, NJ: World Scientific)

[67] Jeffrey G A and Saenger W 1994 Hydrogen Bonding in Biological Structures (Berlin: Springer)

[68] Pakarinen O H, Mativetsky J M, Gulans A, Puska M J, Foster A S and Grütter P 2009 Role of van der Waals forces in the adsorption and diffusion of organic molecules on an insulating surface Phys. Rev. B 80085401

[69] Nony L, Bocquet F, Para F, Chérioux F, Duverger E, Palmino F, Luzet V and Loppacher C 2012 Dipole-driven self-organization of zwitterionic molecules on alkali halide surfaces Beilstein J. Nanotechnol. 3285

[70] Mura M, Sun X, Silly F, Jonkman H T, Briggs G A D, Castell M R and Kantorovich L N 2010 Experimental and theoretical analysis of H-bonded supramolecular assemblies of PTCDA molecules Phys. Rev. B 81195412

[71] Schütte J, Bechstein R, Rohlfing M, Reichling M and Kühnle A 2009 Cooperative mechanism for anchoring highly polar molecules at an ionic surface Phys. Rev. B 80205421

[72] Desiraju G R 2011 A bond by any other name Angew. Chem. Int. Ed. 5052

[73] Israelachvili J 1991 Intermolecular and Surface Forces 2nd edn (San Diego: Academic)

[74] Hunter C A and Sanders J K M 1990 The nature of $\pi-\pi$ interactions J. Am. Chem. Soc. 1125525

[75] Desiraju G R, Ho P S, Kloo L, Legon A C, Marquardt R, Metrangolo P, Politzer P, Resnati G and Rissanen K 2013 Definition of the halogen bond (IUPAC recommendations 2013) Pure Appl. Chem. 851711

[76] Scheiner S 2013 The pnicogen bond: its relation to hydrogen, halogen and other noncovalent bonds Acc. Chem. Res. 46280

[77] Mani D and Arunan E 2013 The X-C...Y (X = O/F, $\mathrm{Y}=\mathrm{O} / \mathrm{S} / \mathrm{F} / \mathrm{Cl} / \mathrm{Br} / \mathrm{N} / \mathrm{P}$ ) 'carbon bond' and hydrophobic interactions Phys. Chem. Chem. Phys. 1514377

[78] Feynman R P 1939 Forces in molecules Phys. Rev. 56340

[79] Feynman R P, Leighton R B and Sands M 1964 The Feynman Lectures on Physics II (Reading, MA: Addison-Wesley)

[80] Hoffmann R 2009 Tip-sample interactions as a function of distance on insulating surfaces Noncontact Atomic Force Microscopy vol 2, ed R W S Morita and E Meyer (Berlin: Wiley)

[81] Rahe P, Nimmrich M and Kühnle A 2012 Substrate templating upon self-assembly of hydrogen-bonded molecular networks on an insulating surface Small 82969

[82] Thiele U 2003 Open questions and promising new fields in dewetting Eur. Phys. J. E 12409

[83] Hoffmann R 1988 A chemical and theoretical way to look at bonding on surfaces Rev. Mod. Phys. 60601

[84] Kühnle A 2009 Self-assembly of organic molecules at metal surfaces Curr. Op. Coll. Interf. Sci. 14157 
[85] Rahe P, Lindner R, Kittelmann M, Nimmrich M and Kühnle A 2012 From dewetting to wetting molecular layers: $\mathrm{C}_{60}$ on $\mathrm{CaCO}_{3}(1014)$ as a case study Phys. Chem. Chem. Phys. 146544

[86] Burke S A, Ji W, Mativetsky J M, Topple J M, Fostner S, Gao H-J, Guo H and Grütter P 2008 Strain induced dewetting of a molecular system: bimodal growth of PTCDA on $\mathrm{NaCl}$ Phys. Rev. Lett. 100186104

[87] Hoffmann R, Kantorovich L N, Baratoff A, Hug H J and Güntherodt H-J 2004 Sublattice identification in scanning force microscopy on alkali halide surfaces Phys. Rev. Lett. 92146103

[88] Lantz M A, Hoffmann R, Foster A S, Baratoff A, Hug H J, Hidber H R and Güntherodt H-J 2006 Sitespecific force-distance characteristics on $\mathrm{NaCl}\left(\begin{array}{lll}0 & 0 & 1\end{array}\right)$ : measurements versus atomistic simulations Phys. Rev. B 74245426

[89] Ruschmeier K, Schirmeisen A and Hoffmann R 2008 Atomic-scale force-vector fields Phys. Rev. Lett. 101156102

[90] Hoffmann R, Lantz M A, Hug H J, van Schendel P J A, Kappenberger P, Martin S, Baratoff A and Güntherodt H-J 2002 Atomic resolution imaging and force versus distance measurements on $\mathrm{KBr}(001)$ using low temperature scanning force microscopy Appl. Surf. Sci. 188238

[91] Foster A S, Barth C, Shluger A L and Reichling M 2001 Unambiguous interpretation of atomically resolved force microscopy images of an insulator Phys. Rev. Lett. 862373

[92] Hoffmann R, Barth C, Foster A S, Shluger A L, Hug H J, Güntherodt H-J, Nieminen R M and Reichling M 2005 Measuring site-specific cluster-surface bond formation J. Am. Chem. Soc. 12717863

[93] Hoffmann R, Weiner D, Schirmeisen A and Foster A S 2009 Sublattice identification in noncontact atomic force microscopy of the $\mathrm{NaCl}(001)$ surface Phys. Rev. B 80115426

[94] Ruschmeier K, Schirmeisen A and Hoffmann R 2009 Sitespecific force-vector field studies of $\operatorname{KBr}(001)$ by atomic force microscopy Nanotechnology 20264013

[95] Venegas de la Cerda M A, Abad J, Madgavkar A, Martrou D and Gauthier S 2008 Step-induced tip polarity reversal investigated by dynamic force microscopy on $\operatorname{KBr}(001)$ Nanotechnology 19045503

[96] Hoffmann R 2001 Magnetic and interatomic forces measured by low temperature scanning force microscopy $P h D$ Thesis University of Basle

[97] Barth C and Henry C R 2007 Surface double layer on (001) surfaces of alkali halide crystals: a scanning force microscopy study Phys. Rev. Lett. 98136804

[98] Bennewitz R, Foster A S, Kantorovich L N, Bammerlin M, Loppacher C, Schär S, Guggisberg M, Meyer E and Shluger A L 2000 Atomically resolved edges and kinks of $\mathrm{NaCl}$ islands on $\mathrm{Cu}(111)$ : experiment and theory Phys. Rev. B 622074

[99] Steiner P, Gnecco E, Krok F, Budzioch J, Walczak L, Konior J, Szymonski M and Meyer E 2011 Atomic-scale friction on stepped surfaces of ionic crystals Phys. Rev. Lett. 106186104

[100] Baetzold R C 1995 Computation of the energetics of surface vacancy and interstitial generation in silver halide Phys. Rev. B 5211424

[101] Kliewer K L and Koehler J S 1965 Space charge in ionic crystals. I. General approach with application to $\mathrm{NaCl}$ Phys. Rev. 140 A1226

[102] Pick H 1965 Struktur von Störstellen in Alkalihalogenidkristallen Springer Tracts Mod. Phys. 381

[103] Garcia R and Perez R 2002 Dynamic atomic force microscopy methods Surf. Sci. Rep. 47197
[104] Giessibl F J 2003 Advances in atomic force microscopy Rev Mod. Phys. 75949

[105] Albrecht T R, Grütter P, Horne D and Rugar D 1991 Frequency-modulation detection using high-Q cantilevers for enhanced force microscope sensitivity J. Appl. Phys. 69668

[106] Dürig U 1999 Relations between interaction force and frequency shift in large-amplitude dynamic force microscopy Appl. Phys. Lett. 75433

[107] Hoffmann R, Baratoff A, Hug H J, Hidber H R, v Löhneysen H and Güntherodt H-J 2007 Mechanical manifestations of rare atomic jumps in dynamic force microscopy Nanotechnology 18395503

[108] Lantz M A, Hug H J, Hoffmann R, van Schendel P J A, Kappenberger P, Martin S, Baratoff A and Güntherodt H-J 2001 Quantitative measurement of short-range chemical bonding forces Science 2912580

[109] Nonnenmacher M, Oboyle M P and Wickramasinghe H K 1991 Kelvin probe force microscopy Appl. Phys. Lett. 582921

[110] Sadewasser S and Glatzel T (ed) 2012 Kelvin Probe Force Microscopy (Berlin: Springer)

[111] Mohn F, Gross L, Moll N and Meyer G 2012 Imaging the charge distribution within a single molecule Nat. Nanotechnol. 7227

[112] Milde P 2011 Visualisation of local charge densities with Kelvin probe force microscopy $P h D$ Thesis TU Dresden

[113] Sadeghi A, Baratoff A and Goedecker S 2013 Electrostatic interactions with dielectric samples in scanning probe microscopies Phys. Rev. B 88035436

[114] Burke S A, LeDue J M, Miyahara Y, Topple J M, Fostner S and Grütter P 2009 Determination of the local contact potential difference of PTCDA on $\mathrm{NaCl}$ : a comparison of techniques Nanotechnology 20264012

[115] Wigner E and Bardeen J 1935 Theory of the work functions of monovalent metals Phys. Rev. 4884

[116] Smoluchowski R 1941 Anisotropy of the electronic work function of metals Phys. Rev. 60661

[117] Milde P, Zerweck U, Eng L M, Abel M, Giovanelli L, Nony L, Mossoyan M, Porte L and Loppacher C 2008 Interface dipole formation of different $\mathrm{ZnPcCl}(8)$ phases on $\operatorname{Ag}\left(\begin{array}{lll}1 & 1 & 1\end{array}\right)$ observed by Kelvin probe force microscopy Nanotechnology 19305501

[118] Iwamoto M and Itoh E 1998 Nano-electrostatic phenomena in Langmuir-Blodgett films Thin Solid Films 33115

[119] Kitamura M and Arakawa Y 2008 Pentacene-based organic field-effect transistors J. Phys.: Condens. Matter 20184011

[120] Neff J L, Götzen J, Li E, Marz M and Hoffmann-Vogel R 2012 Molecular resolution imaging of pentacene on $\mathrm{KCl}(0001)$ Beilstein J. Nanotechnol. 3186

[121] Dinca L E, Fu C, MacLeod J M, Lipton-Duffin J, Brusso J L, Szakacs C E, Ma D, Perepichka D F and Rosei F 2013 Unprecedented transformation of tetrathienoanthracene into pentacene on Ni(1 11) ACS Nano 71652

[122] Kawai S, Eren B, Marot L and Meyer E 2014 Graphene synthesis via thermal polymerization of aromatic quinone molecules ACS Nano 85932

[123] Nabok D, Puschnig P, Ambrosch-Draxl C, Werzer O, Resel R and Smilgies D-M 2007 Crystal and electronic structures of pentacene thin films from grazing-incidence $\mathrm{x}$-ray diffraction and first-principles calculations Phys. Rev. B 76235322

[124] Campbell R B, Robertson J M and Trotter J 1961 Crystal and molecular structure of pentacene Acta Crystallogr. 14705

[125] Ambrosch-Draxl C, Nabok D, Puschnig P and Meisenbichler C 2009 The role of polymorphism in organic thin films: oligoacenes investigated from first principles New. J. Phys. 11125010 
[126] Mattheus C C, Dros A B, Baas J, Oostergetel G T, Meetsma A, de Boer J L and Palstra T T M 2003 Identification of polymorphs of pentacene Synth. Met. 138475

[127] Kiyomura T, Nemoto T, Yoshida K, Minari T, Kurata H and Isoda S 2006 Epitaxial growth of pentacene thin-film phase on alkali halides Thin Solid Films 515810

[128] Kiyomura T, Nemoto T, Ogawa T, Minari T, Yoshida K, Kurata H and Isoda S 2006 Thin-film phase of pentacene film formed on $\mathrm{KCl}$ by vacuum deposition Japan. J. Appl. Phys. 45401

[129] Kakudate T, Yoshimoto N, Kawamura K and Saito Y 2007 Observation of epitaxial growth of pentacene thin films on $\mathrm{KCl}$ substrate by x-ray diffractometry J. Cryst. Growth 30627

[130] Ruiz R, Nickel B, Koch N, Feldman L C, Haglund R F, Kahn A and Scoles G 2003 Pentacene ultrathin film formation on reduced and oxidized Si surfaces Phys. Rev. B 67125406

[131] Zorba S, Shapir Y and Gao Y 2006 Fractal-mound growth of pentacene thin films Phys. Rev. B 74245410

[132] Stadlober B, Haas U, Maresch H and Haase A 2006 Growth model of pentacene on inorganic and organic dielectrics based on scaling and rate-equation theory Phys. Rev. B 74165302

[133] Ruiz R, Nickel B, Koch N, Feldman L C, Haglund R F, Kahn A, Family F and Scoles G 2003 Dynamic scaling, island size distribution and morphology in the aggregation regime of submonolayer pentacene films Phys. Rev. Lett. 91136102

[134] Lukas S, Witte G and Wöll C 2001 Novel mechanism for molecular self-assembly on metal substrates: unidirectional rows of pentacene on $\mathrm{Cu}(110)$ produced by a substrate-mediated repulsion Phys. Rev. Lett. 88028301

[135] Kawai S, Pawlak R, Glatzel T and Meyer E 2011 Systematic measurement of pentacene assembled on $\mathrm{Cu}\left(\begin{array}{lll}1 & 1 & 1\end{array}\right)$ by bimodal dynamic force microscopy at room temperature Phys. Rev. B 84085429

[136] Götzen J, Lukas S, Birkner A and Witte G 2011 Absence of template induced ordering in organic multilayers: the growth of pentacene on a $\mathrm{Cu}(221)$ vicinal surface Surf. Sci. 605577

[137] Käfer D, Ruppel L and Witte G 2007 Growth of pentacene on clean and modified gold surfaces Phys. Rev. B 75085309

[138] France C B, Schroeder P G, Forsythe J C and Parkinson B A 2003 Scanning tunneling microscopy study of the coverage-dependent structures of pentacene on $\mathrm{Au}(111)$ Langmuir 941274

[139] Menozzia C, Corradini V, Cavallini M, Biscarini F, Betti M G and Mariani C 2003 Pentacene self-aggregation at the $\mathrm{Au}(110)-(1 \times 2)$ surface: growth morphology and interface electronic states Thin Solid Films 428227

[140] Götzen J, Käfer D, Wöll C and Witte G 2010 Growth and structure of pentacene films on graphite: weak adhesion as a key for epitaxial film growth Phys. Rev. B 81085440

[141] Cheng H-L, Mai Y-S, Chou W-Y, Chang L-R and Liang X-W 2007 Thickness-dependent structural evolutions and growth models in relation to carrier transport properties in polycrystalline pentacene thin film Adv. Funct. Mater. 173639

[142] Mishima R, Loan N T and Tada H 2012 Electrostatic properties of organic monolayers on silicon oxides studied by kelvin probe force microscopy Jpn. J. Appl. Phys. 51045702

[143] Kimura T, Miyato Y, Kobayashi K, Yamada H and Matsushige K 2012 Investigations of local electrical characteristics of a pentacene thin film by point-contact current imaging atomic force microscopy Japan. J. Appl. Phys. 51 08KB05
[144] Yogev S, Matsubara R, Nakamura M, Zschieschang U, Klauk H and Rosenwaks Y 2013 Fermi level pinning by gap states in organic semiconductors Phys. Rev. Lett. 110036803

[145] Kroto H W, Heath J R, O'Brien S C, Curl R F and Smalley R E 1985 C $_{60}$ : buckminsterfullerene Nature 318162

[146] Girifalco L 1992 Molecular properties of $\mathrm{C}_{60}$ in the gas and solid phases J. Phys. Chem. 96858

[147] Moreno C, Stetsovych O, Shimizu T K and Custance O 2015 Imaging three-dimensional surface objects with submolecular resolution by atomic force microscopy Nano Lett. 152257

[148] Altman E I and Colton R J 1992 Nucleation, growth and structure of fullerene films on Au(1 111$)$ Surf. Sci. 27949

[149] Altman E I and Colton R J 1993 The interaction of $\mathrm{C}_{60}$ with noble-metal surfaces Surf. Sci. 29513

[150] Thundat T, Warmack R J, Ding D and Compton R N 1993 Atomic force microscope investigation of $\mathrm{C}_{60}$ adsorbed on silicon and mica Appl. Phys. Lett. 63891

[151] Shi X, Caldwell W B, Chen K and Mirkin C A 1994 A well-defined surface-confinable fullerene: monolayer selfassembly on $\mathrm{Au}(111$ 1) J. Am. Chem. Soc. 11611598

[152] Schwarz U D, Allers W, Gensterblum G, Pireaux J-J and Wiesendanger R 1995 Growth of $\mathrm{C}_{60}$ thin films on $\mathrm{GeS}(001)$ studied by scanning force microscopy Phys. Rev. B 525967

[153] Tanigaki K, Kuroshima S, Fujita J and Ebbesen T W 1993 Crystal growth of $\mathrm{C}_{60}$ thin films on layered substrates Appl. Phys. Lett. 632351

[154] Tanigaki K, Kuroshima S and Ebbesen T W 1995 Crystalgrowth and structure of fullerene thin-films Thin Solid Films 257154

[155] Yase K, Ara-Kato N, Hanada T, Takiguchi H, Yoshida Y, Back G, Abe K and Tanigaki N 1998 Aggregation mechanism in fullerene thin films on several substrates Thin Solid Films 331131

[156] Moriarty P J 2010 Fullerene adsorption on semiconductor surfaces Surf. Sci. Rep. 65175

[157] Böhringer M, Schneider W-D and Berndt R 1998 Scanning tunneling microscope-induced molecular motion and its effect on the image formation Surf. Sci. 40872

[158] Girard C, Lambin P, Dereux A and Lucas A A 1994 van der waals attraction between two $\mathrm{C}_{60}$ fullerene molecules and physical adsorption of $\mathrm{C}_{60}$ on graphite and other substrates Phys. Rev. B 4911425

[159] Jung S C and Kang M H 2008 First-principles study of a single $\mathrm{C}_{60}$ cluster adsorbed on $\mathrm{KBr}(100)$ Surf. Sci. $\mathbf{6 0 2} 1916$

[160] dos Santos Pinheiro L and Mendes Filho J 2013 Exchange experiments of $\mathrm{C}_{60}$ and halides on a $\mathrm{Au}(1111)$ surface Appl. Surf. Sci. 280312

[161] Mativetsky J M, Fostner S, Burke S A and Grütter P 2008 The role of charge-induced defects in the growth of gold on an alkali halide surface Surf. Sci. 602 L21

[162] Kobayashi K, Yamada H, Horiuchi T and Matsushige K 1999 Investigations of $\mathrm{C}_{60}$ molecules deposited on $\mathrm{Si}\left(\begin{array}{lll}1 & 1 & 1\end{array}\right)$ by noncontact atomic force microscopy Appl. Surf. Sci. 140281

[163] Such B, Trevethan T, Glatzel T, Kawai S, Zimmerli L, Meyer E, Shluger A L, Amijs C H M, de Mendoza P and Echavarren A M 2010 Functionalized truxenes: adsorption and diffusion of single molecules on the $\operatorname{KBr}(001)$ surface ACS Nano 43429

[164] Ittermann B, Hoffmann-Vogel R, Behrens L and Baratoff A 2013 Simulations of metastable states near the apex of a force microscope tip interacting with an ionic crystalline surface Phys. Rev. B 87195437 
[165] Böhringer M, Morgenstern K, Schneider W-D and Berndt R 2000 Reversed surface corrugation in STM images on $\mathrm{Au}(111$ ) by field-induced lateral motion of adsorbed molecules Surf. Sci. 45737

[166] Martsinovich N and Kantorovich L 2009 Modelling the manipulation of $\mathrm{C}_{60}$ on the $\mathrm{Si}(001)$ surface performed with NC-AFM Nanotechnology 20135706

[167] Trevethan T, Such B, Glatzel T, Kawai S, Shluger A L, Meyer E, de Mendoza P and Echavarren A M 2011 Organic molecules reconstruct nanostructures on ionic surfaces Small 71264

[168] Rosei F, Schunack M, Jiang P, Gourdon A, Laegsgaard E, Stensgaard I, Joachim C and Besenbacher F 2002 Organic molecules acting as templates on metal surfaces Science 296328

[169] Mativetsky J M, Burke S A, Hoffmann R, Sun Y and Grütter P 2004 Molecular resolution imaging of $\mathrm{C}_{60}$ on $\mathrm{Au}\left(\begin{array}{lll}1 & 1 & 1\end{array}\right)$ by non-contact atomic force microscopy Nanotechnology 15 S40

[170] Loske F, Rahe P and Kühnle A 2009 Contrast inversion in non-contact atomic force microscopy imaging of $\mathrm{C}_{60}$ molecules Nanotechnology 20264010

[171] Arai T and Tomitori M 2000 Bias dependence of Si (111) $7 \times 7$ images observed by noncontact atomic force microscopy Appl. Surf. Sci. 157207

[172] Rahe P, Bechstein R, Schütte J, Ostendorf F and Kühnle A 2008 Repulsive interaction and contrast inversion in noncontact atomic force microscopy imaging of adsorbates Phys. Rev. B 77195410

[173] Pawlak R, Kawai S, Fremy S, Glatzel T and Meyer E 2012 High-resolution imaging of $\mathrm{C}_{60}$ molecules using tuningfork-based non-contact atomic force microscopy J. Phys.: Condens. Matter 24084005

[174] Baratoff A 2000 unpublished

[175] Giessibl F J and Bielefeldt H 2000 Physical interpretation of frequency-modulation atomic force microscopy Phys. Rev B 619968

[176] Böhringer M, Morgenstern K, Schneider W-D, Berndt R, Mauri F, Vita A D and Car R 1999 Two-dimensional selfassembly of supramolecular clusters and chains Phys. Rev Lett. 83324

[177] Rey C, Garcia-Rodeja J, Gallego L J and Alonso J A 1997 Clusters and layers of $\mathrm{C}_{60}$ molecules supported on a graphite substrate Phys. Rev. B 557190

[178] Kervyn S, Kalashnyk N, Riello M, Moreton B, Tasseroul J, Wouters J, Jones T S, Vita A D, Costantini G and Bonifazi D 2013 'Magic' surface clustering of borazines driven by repulsive intermolecular forces Angew. Chem. Int. Ed. $\mathbf{5 2} 7410$

[179] Grobis M, Lu X and Crommie M F 2002 Local electronic properties of a molecular monolayer: $\mathrm{C}_{60}$ on $\mathrm{Ag}(001)$ Phys. Rev. B $66161408 \mathrm{R}$

[180] Gimzewski J K, Modesti S and Schlittler R R 1994 Cooperative self-assembly of $\mathrm{Au}$ atoms and $\mathrm{C}_{60}$ on $\mathrm{Au}(110)$ surfaces Phys. Rev. Lett. 721036

[181] Hsu C-L and Pai W W 2003 Aperiodic incommensurate phase of a $\mathrm{C}_{60}$ monolayer on $\mathrm{Ag}(100)$ Phys. Rev. B 68245414

[182] Gaisch R, Berndt R, Gimzewski J K, Reihl B, Schlittler R R, Schneider W D and Tschudy M 1993 Internal structure of $\mathrm{C}_{60}$ fullerene molecules as revealed by low-temperature stm Appl. Phys. A 57207

[183] Larsson J A, Elliott S D, Greer J C, Repp J, Meyer G and Allenspach R 2008 Orientation of individual $\mathrm{C}_{60}$ molecules adsorbed on $\mathrm{Cu}\left(\begin{array}{lll}1 & 1 & 1\end{array}\right)$ : low-temperature scanning tunneling microscopy and density functional calculations Phys. Rev. B 77115434

[184] Pawlak R, Kawai S, Fremy S, Glatzel T and Meyer E 2011 Atomic-scale mechanical properties of orientated
$\mathrm{C}_{60}$ molecules revealed by noncontact atomic force microscopy ACS Nano 56349

[185] Chiutu C, Sweetman A M, Lakin A J, Stannard A, Jarvis S, Kantorovich L, Dunn J L and Moriarty P 2012 Precise orientation of a single $\mathrm{C}_{60}$ molecule on the tip of a scanning probe microscope Phys. Rev. Lett. 108268302

[186] Fendrich M, Wagner T, Stöhr M and Möller R 2006 Hindered rotation of a copper phthalocyanine molecule on $\mathrm{C}_{60}$ : experiments and molecular mechanics calculations Phys. Rev. B 73115433

[187] Stöhr M, Wagner T, Gabriel M, Weyers B and Möller R 2002 Direct observation of hindered eccentric rotation of an individual molecule: Cu-phthalocyanine on $\mathrm{C}_{60}$ Phys. Rev. B 65033404

[188] Burke S A, Mativetsky J M, Fostner S and Grütter P 2007 $\mathrm{C}_{60}$ on alkali halides: epitaxy and morphology studied by noncontact AFM Phys. Rev. B 76035419

[189] Körner M, Loske F, Einax M, Kühnle A, Reichling M and Maass P 2011 Second-layer induced island morphologies in thin-film growth of fullerenes Phys. Rev. Lett. 107016101

[190] Shin H, O’Donnell S E, Reinke P, Ferralis N, Schmid A K, Li H I, Novaco A D, Bruch L W and Diehl R D 2010 Floating two-dimensional solid monolayer of $\mathrm{C}_{60}$ on graphite Phys. Rev. B 82235427

[191] Einax M, Dieterich W and Maass P 2013 Colloquium: Cluster growth on surfaces: densities, size distributions and morphologies Rev. Mod. Phys. 85921

[192] Liu H and Reinke P $2006 \mathrm{C}_{60}$ thin film growth on graphite: coexistence of spherical and fractal-dendritic islands J. Chem. Phys. 124164707

[193] Seydel E, Hoffmann-Vogel R and Marz M 2017 Growth of $\mathrm{C}_{60}$ on highly oriented pyrolytic graphite (HOPG) surfaces studied at low temperatures unpublished

[194] Chen W, Zhang H L, Xu H, Tok E S, Loh K P and Wee A T S $2006 \mathrm{C}_{60}$ on SiC nanomesh J. Phys. Chem. B 11021873

[195] Wang S, Zhang Y, Abidi N and Cabrales L 2009 Wettability and surface free energy of graphene films Langmuir 2511078

[196] Sasahara A, Uetsuka H and Onishi H 2001 Noncontact atomic force microscope topography dependent on the electrostatic dipole field of individual molecules Phys. Rev. B 64121406

[197] Stöhr M, Gabriel M and Möller R 2002 Analysis of the three-dimensional structure of a small crystallite by scanning tunneling microscopy: multilayer films of 3,4,9,10-perylenetetracarboxylic-dianhydride (PTCDA) on $\mathrm{Cu}(110)$ Europhys. Lett. 59423

[198] Tautz F 2007 Structure and bonding of large aromatic molecules on noble metal surfaces: the example of PTCDA Prog. Surf. Sci. 82479

[199] Tautz F, Eremtchenko M, Schaefer J, Sokolowski M, Shklover V, Glöckler K and Umbach E 2002 A comparison of the chemisorption behaviour of PTCDA on different Ag surfaces Surf. Sci. 502-503 176

[200] Burke S A, LeDue J M, Topple J M, Fostner S and Grütter P 2009 Relating the functional properties of an organic semiconductor to molecular structure by nc-AFM $A d v$ Mater 212029

[201] Schlettwein D, Back A, Schilling B, Fritz T and Armstrong N R 1998 Ultrathin films of perylenedianhydride and perylenebis(dicarboximide) dyes on $(001)$ alkali halide surfaces Chem. Mater. 10601

[202] Wagner T, Bannani A, Bobisch C, Karacuban H, Stöhr M, Gabriel M and Möller R 2004 Growth of 3,4,9,10-perylenetetracarboxylic-dianhydride crystallites on noble metal surfaces Org. Electron. 535

[203] Yim S, Kim K-I and Jones T S 2007 Growth morphology of perylene-3,4,9,10-tetracarboxylic dianhydride (PTCDA) 
thin films: influence of intermolecular interactions and step-edge barriers J. Phys. Chem. C 11110993

[204] Karacuban H, Koch S, Fendrich M, Wagner T and Möller R 2011 PTCDA on $\mathrm{Cu}(111)$ partially covered with $\mathrm{NaCl}$ Nanotechnology 22295305

[205] Krause B, Dürr A C, Ritley K, Schreiber F, Dosch H and Smilgies D 2002 Structure and growth morphology of an archetypal system for organic epitaxy: PTCDA on Ag(1 11 1) Phys. Rev. B 66235404

[206] Krause B, Dürr A C, Schreiber F, Dosch H and Seeck O H 2003 Thermal stability and partial dewetting of crystalline organic thin films: 3,4,9,10-perylenetetracarboxylic dianhydride on $\operatorname{Ag}(1111)$ J. Chem. Phys. 1193429

[207] Braun D-A, Langewisch G, Fuchs H and Schirmeisen A 2010 Force field experiments of an epitaxial superstructure of 3,4,9,10-perylenetetra-carboxylic-dianhydride on $\mathrm{Ag}\left(\begin{array}{lll}1 & 1 & 1\end{array}\right)$ J. Vac. Sci. Technol. B 28 C4B6

[208] Loppacher C, Zerweck U, Eng L M, Gemming S, Seifert G, Olbrich C, Morawetz K and Schreiber M 2006 Adsorption of PTCDA on a partially $\mathrm{KBr}$ covered $\mathrm{Ag}(111)$ substrate Nanotechnology 171568

[209] Mativetsky J M, Burke S A, Fostner S and Grütter P 2007 Templated growth of 3,4,9,10-perylenetetracarboxylic dianhydride molecules on a nanostructured insulator Nanotechnology 18105303

[210] Godlewski S, Tekiel A, Piskorz W, Zasada F, PrauznerBechcicki J S, Sojka Z and Szymonski M 2012 Supramolecular ordering of PTCDA molecules: the key role of dispersion forces in an unusual transition from physisorbed into chemisorbed state ACS Nano 68536

[211] Such B, Weiner D, Schirmeisen A and Fuchs H 2006 Influence of the local adsorption environment on the intramolecular contrast of organic molecules in noncontact atomic force microscopy Appl. Phys. Lett. 89093104

[212] Huber F, Matencio S, Weymouth A J, Ocal C, Barrena E and Giessibl F J 2015 Intramolecular force contrast and dynamic current-distance measurements at room temperature Phys. Rev. Lett. 115066101
[213] Moll N, Gross L, Mohn F, Curioni A and Meyer G 2012 A simple model of molecular imaging with noncontact atomic force microscopy New J. Phys. 14083023

[214] Langewisch G, Falter J, Schirmeisen A and Fuchs H 2014 Influence of the adsorption geometry of PTCDA on $\operatorname{Ag}\left(\begin{array}{lll}1 & 1 & 1)\end{array}\right.$ on the tip-molecule forces in non-contact atomic force microscopy Beilstein J. Nanotechnol. 598

[215] Wagner C, Fournier N, Tautz F S and Temirov R 2012 Measurement of the binding energies of the organicmetal perylene-teracarboxylic-dianhydride/Au( 1111$)$ bonds by molecular manipulation using an Atomic Force Microscope Phys. Rev. Lett. 109076102

[216] Wagner C, Fournier N, Tautz F S and Temirov R 2014 The role of surface corrugation and tip oscillation in singlemolecule manipulation with a non-contact atomic force microscope Beilstein J. Nanotechnol. 5202

[217] Lange M, van Vörden D and Möller R 2012 A measurement of the hysteresis loop in force-spectroscopy curves using a tuning-fork atomic force microscope Beilstein $J$. Nanotechnol. 3207

[218] Langewisch G, Kamiński W, Braun D-A, Möller R, Fuchs H, Schirmeisen A and Perez R 2012 Understanding dissipative tip-molecule interactions with submolecular resolution on an organic adsorbate Small 8602

[219] Dienel T, Loppacher C, Mannsfeld S C B, Forker R and Fritz T 2008 Growth-mode-induced narrowing of optical spectra of an organic adlayer Adv. Mater. 20959

[220] Le Moal E, Müller M, Bauer O and Sokolowski M 2011 Stable and metastable phases of PTCDA on epitaxial $\mathrm{NaCl}$ films on $\mathrm{Ag}(100)$ Phys. Rev. B 82045301

[221] Seidel C, Atwater C, Liu X D, Ellerbrake R and Fuchs H 1997 A combined STM, LEED and molecular modelling study of PTCDA grown on $\operatorname{Ag}\left(\begin{array}{lll}1 & 1 & 0\end{array}\right)$ Surf. Sci. 371123

[222] Toton D, Godlewski S, Goryl G, Kolodziej J J, Kantorovich L and Szymonski M 2011 Architecture of PTCDA molecular structures on a reconstructed $\operatorname{InSb}\left(\begin{array}{ll}0 & 0\end{array}\right)$ surface Phys. Rev. B 83235431

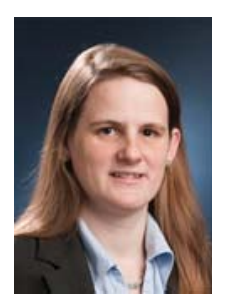

Regina Hoffmann-Vogel works at Karlsruhe Institute of Technology. She received her Diploma in physics from the University of Karlsruhe, her diplôme d'ingénieur from the Ecole Nationale Superieure de Physique de Grenoble, and her Ph. D. degree in Physics from University of Basle in 2001. Before joining Karlsruhe Institute of Technology, she worked at McGill university, Montreal, where she investigated molecules on insulating surfaces. A starting grant financed by the European Commission's European Research Council (ERC) allowed her to start independent research at Karlsruhe. Her current research focuses on high-resolution scanning force microscopy studies of surfaces as well as the formation of metallic nanocontacts by electromigration. She has authored or co-authored more than 50 articles in peer-reviewed journals and has delivered over 60 invited talks. 